\title{
DIOPHANTINE APPROXIMATION OF COMPLEX NUMBERS
}

\author{
BY
}

ASMUS L. SCHMIDT

University of Copenhagen, Denmark

\section{Contents}

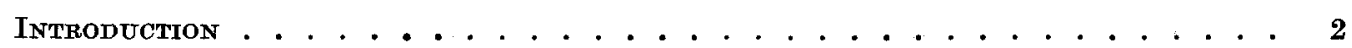

Chapter 1. Farey sets

1.1. Some basic notation. . . . . . . . . . . . . . . . . . . . . . . 4 4

1.2. Farey sets. . . . . . . . . . . . . . . . . . . . . 6

1.3. A generating procedure of Farey sets . . . . . . . . . . . . . . . . . 9

1.4. Dual Farey sets. . . . . . . . . . . . . . . . . . . . . . 13

Chapter 2. Regular and dUatixy RegUlar chatns

2.1. Representation of complex numbers . . . . . . . . . . . . . . . . . . . . . 14

2.2. Equivalence . . . . . . . . . . . . . . . . . . . . . 19

2.3. Approximation theorems. . . . . . . . . . . . . . . . . . . 21

Chapter 3. Periodic chatns

3.1. Periodic chains . . . . . . . . . . . . . . . . . . . . . . . . . 24

3.2. Purely periodic chains . . . . . . . . . . . . . . . . . . . . . . 29

3.3. Inverse periods . . . . . . . . . . . . . . . . . . . . . . . . 31

3.4. Special quadratic surds. The Pellian equation. . . . . . . . . . . . . . . 32

Chapter 4. $C$-RegUlar and $C$-DUALLY RegUlar CONTINUEd Fractions

4.1. Introduction . . . . . . . . . . . . . . . . . . . . . . . . 35

4.2. $C$-equivalence and $C$-duality . . . . . . . . . . . . . . . . 37

4.3. $C$-convergents and $C$-dual convergents . . . . . . . . . . . . . . . 38

4.4. The $C$-approximation constant . . . . . . . . . . . . . . . . 39

4.5. Ergodic theory . . . . . . . . . . . . . . . . . . . . 4I

Chapter 5. $C$-minimum of binary quadratic forms

5.1. Indefinite quadratic forms . . . . . . . . . . . . . . . . . . 43

5.2. A diophantine equation . . . . . . . . . . . . . . . . . . 45

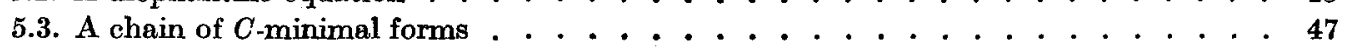

5.4. The main theorem on the $C$-minimum of forms . . . . . . . . . . . . . . . . 52

5.5. The main theorem on the $C$-approximation constant . . . . . . . . . . . . . . 54

5.6. Other methods . . . . . . . . . . . . . . . . . . . . . . 55

1-752903 Acta mathematica 134. Imprimé le 30 Juillet 1975 
Chapter 6. Complex binary quadratic forms

6.1. Reduction theory . . . . . . . . . . . . . . . . . . . . . . . . . . 56

6.2. Minimum of quadratic forms . . . . . . . . . . . . . . . . . . . . . 63

6.3. Regular double chains $\Pi T_{n}$ with $K<2 \quad$. . . . . . . . . . . . . . . . . . . . . . . . . 65

6.4. The main theorem on the minimum of quadratic forms _ . . . . . . . . . . . . . . . . 78

6.5. The main theorem on the approximation constant . . . . . . . . . . . . . . . 81

REFERENCES ............................. 84

\section{Introduction}

Several attempts have been made during the last 100 years to develop for complex numbers an algorithm having the properties that the regular continued fraction algorithm is known to possess in the real case, due to results of Euler, Lagrange, Legendre, Gauss, Galois, Serret, Markoff, and Hurwitz.

The most significant of such previous attempts are those of A. Hurwitz [18], J. Hurwitz [20], A. Auric [1], L. R. Ford [12], J. W. S. Cassels, W. Ledermann, K. Mahler [5], W. J. Leveque [22, 23], G. Poitou [28], and A. L. Schmidt [33]. However, it is a common feature of all these approaches that only very few of the nice properties of regular continued fractions are carried over.

It is the purpose of the present paper to develop in the Gaussian case two new kinds of algorithm, regular chains and dually regular chains, based on the concepts of Farey sets and dual Farey sets to be presented in chapter 1. As is pointed out, Farey sets appear to be a natural extension of the well-known circles and mesh triangles of L. R. Ford [13]. For this chapter we presuppose some knowledge of Farey triangles (sections 2-5 of [33]).

It is shown in chapter 2 that the representation of complex irrational numbers $\xi$ by regular and dually regular chains-ch $\xi$ and $\mathrm{ch}^{*} \xi$-is essentially unique, and that the theorem of Serret about equivalence extends in a natural way. Also every fair approximant $p / q$ (with $p, q \in \mathbf{Z}[i]$ ) of $\xi$ will appear as a convergent of $\operatorname{ch} \xi$ and of $\operatorname{ch}^{*} \xi$, thus extending a theorem of Legendre.

The classical theorems of Euler, Lagrange, and Galois about periodic and purely periodic regular continued fractions are extended in chapter 3 and used to give an effective solution of the complex Pellian equation.

Chapter 4 is devoted to a study of $\operatorname{ch} \xi_{0}$ and $\operatorname{ch}^{*} \xi_{0}$ for $\xi_{0}=\frac{1}{2}\left(1+i \alpha_{0}\right)$, where $\alpha_{0} \in \mathbf{R} \backslash \mathbf{Q}$, and of the corresponding $C$-regular and $C$-dually regular continued fractions (4.2) and (4.2*) of $\alpha_{0}$. These continued fractions, though sharing also the more subtle properties of regular continued fractions (an intrinsic characterization of convergents, a theory of $C$-approximation constants $<2$ and an ergodic theory in complete analogy with the corresponding 
theorems for regular: continued fractions by Legendre, Markoff-Hurwitz and C. RyllNardzewski), seem, somehow, to have escaped earlier notice.

In chapter 5 the theory of $C$-minimum of real binary quadratic forms and the corresponding theory of $C$-approximation constants is treated in complete analogy with the exposition by J. W. S. Cassels [4] on the Markoff chain.

In a separate paper $\mathrm{I}$ shall deduce the properties of the $C$-minimal forms $g_{\Lambda}$ or $h_{\mathrm{M}}$ of chapter 5, by putting these in 1-1 correspondance to Markoff-symbols representing periods of even or odd lengths, respectively.

Chapter 6 contains first of all a reduction theory of complex binary quadratic forms similar to that of C. F. Gauss [16] for real indefinite binary quadratic forms. Apparently the only reduction theory of complex binary quadratic forms existing is the rather crude one contained in the famous paper of P. G. L. Dirichlet [10] on what is now known as "Dirichlet fields".

An important application of this reduction theory is the complete determination--in Theorem 6.6-of all complex binary quadratic forms $\Phi$ with $\sqrt{|D|} / \mu<2$, where $D$ and $\mu$ denote the discriminant and minimum of $\Phi$.

Also chapter 6 contains the complete determination-in Theorem 6.7-of all complex irrationals $\xi$ with approximation constant $C(\xi)<2$.

Contributors to the early development of these theorems are L. R. Ford [13] and O. Perron [25, 26], who both determined the first minimum. Later J. W. S. Cassels [3] and A. L. Schmidt [33] proved the isolation of the first minimum, and J. W. S. Cassels [3] also indicated the forms $G, \bar{G}$ of Theorem 6.6. Very recently L. Ya. Vulakh [36] determined the minimum of the forms $G_{\Lambda}$ of Theorem 6.6 (in an equivalent form), and gave also a brief indication of a proof of the symmetric case of that theorem, without, however, being explicit about the isolation technique involved.

In a separate paper I shall use the machinery of chapter 6 also to study the minimum of complex ternary and quaternary quadratic forms.

Also I announce a forthcoming paper on "Hurwitzian chains", including (for $a, b \in \mathbf{N}$ )

$$
\begin{gathered}
\operatorname{ch} \exp [1 /(a-i b)]=\left.V_{3} \overline{V_{n}^{2 b n+b-2}} \bar{E}_{n+2} \bar{V}_{n}^{2 a n+a-1} V_{n+2} \vec{C}\right|_{n=0} ^{\infty}, \\
\operatorname{ch} \exp [1 /(-i b)]=\left.V_{3} \overline{V_{3}^{2 b n+b-2}} \vec{C}^{2}\right|_{n=0} ^{\infty},
\end{gathered}
$$

which extend the classical formula of Euler (cf. [27]),

$$
\exp [\mathbf{1} / a]=[\overrightarrow{1,2 a n+a-1,1,}]_{n=0}^{\infty} \text {. }
$$

Finally I announce a paper on the approximation of complex numbers by numbers 
from the field $\mathbf{Q}(\sqrt{-1})$. By combining the methods of the present paper with those of [33], I shall prove that the set of approximation constants has

$$
((1588626+30690 \sqrt{1085}) / 1205821)^{1 / 2}=1.4682 \ldots
$$

as the smallest limit point. There are infinitely many approximation constants below this point, the four smallest being

$$
\frac{1}{2} \sqrt{5}, \sqrt{2}, \sqrt{\frac{1085}{509}}, \sqrt{\frac{36860}{17099}}
$$

\section{Chapter 1}

Farey sets

\subsection{Some basic notation}

Let

$$
\begin{aligned}
& \mathfrak{J}=\{z=x+i y \mid y \geqslant 0\} \cup\{\infty\}, \\
& \mathfrak{J}^{*}=\left\{z=x+i y \mid 0 \leqslant x \leqslant 1, y \geqslant\left(x-x^{2}\right)^{1 / 2}\right\} \cup\{\infty\} .
\end{aligned}
$$

The sets $\mathfrak{J}, \mathfrak{J}^{*}$ and their subdivisions into

$$
\begin{aligned}
& \boldsymbol{J}=\mathfrak{\vartheta}_{1} \cup \mathfrak{\vartheta}_{2} \cup \mathfrak{\vartheta}_{3} \cup \mathcal{E}_{1} \cup \mathcal{E}_{2} \cup \mathcal{E}_{3} \cup \mathrm{C}, \\
& \mathcal{J}^{*}=\mathfrak{\vartheta}_{1}^{*} \cup \mathfrak{\vartheta}_{2}^{*} \cup \mathfrak{\vartheta}_{3}^{*} \cup \mathcal{C}^{*},
\end{aligned}
$$

are shown in Fig. 1, Fig. $1^{*}$, respectively. All regions are bounded by straight lines or circles with radii $1 / 2$ (or part thereof), and are supposed to be closed; $\infty \in \mathfrak{V}_{1}, \mathcal{E}_{2}, \mathcal{E}_{3}, \mathfrak{\vartheta}_{1}^{*}$.

The matrices $V_{1}, V_{2}, V_{3}, E_{1}, E_{2}, E_{3}, C, S, I$ are defined as follows,

$$
\begin{array}{lll}
V_{1}=\left(\begin{array}{ll}
1 & i \\
0 & 1
\end{array}\right), & V_{2}=\left(\begin{array}{rr}
1 & 0 \\
-i & 1
\end{array}\right), & V_{3}=\left(\begin{array}{cc}
1-i & i \\
-i & 1+i
\end{array}\right), \\
E_{1}=\left(\begin{array}{cc}
1 & 0 \\
1-i & i
\end{array}\right), & E_{2}=\left(\begin{array}{cc}
1 & -1+i \\
0 & i
\end{array}\right), & E_{3}=\left(\begin{array}{ll}
i & 0 \\
0 & 1
\end{array}\right), \\
C=\left(\begin{array}{cc}
1 & -1+i \\
1-i & i
\end{array}\right), & S=\left(\begin{array}{cc}
0 & -1 \\
1 & -1
\end{array}\right), & I=\left(\begin{array}{ll}
1 & 0 \\
0 & 1
\end{array}\right) .
\end{array}
$$

For any invertible Matrix $M$,

$$
M=\left(\begin{array}{ll}
a & b \\
c & d
\end{array}\right)
$$



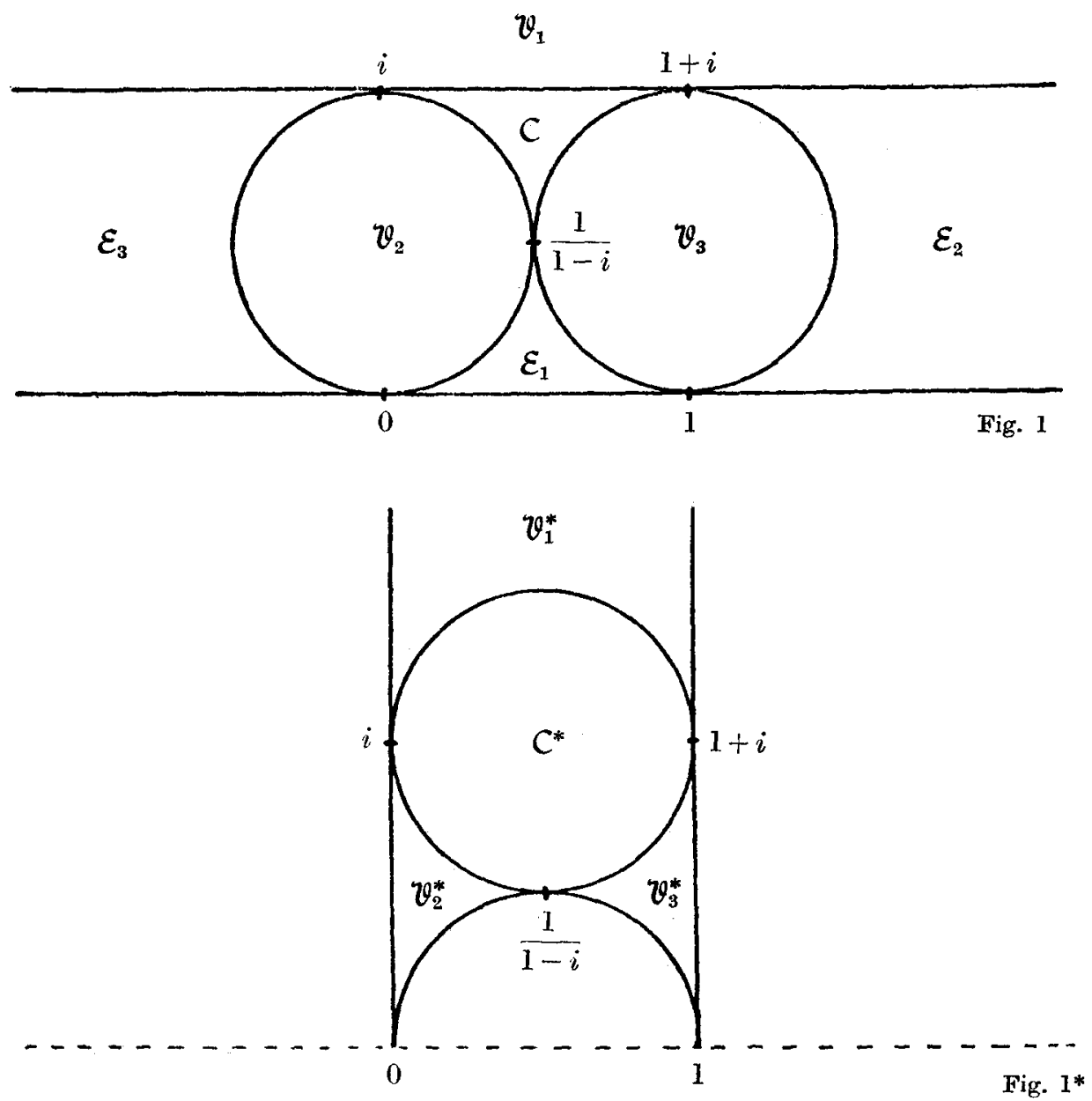

with elements in $\mathbf{C}$, let $m$ denote the corresponding homographic map

$$
m: z \mapsto \frac{a z+b}{c z+d}
$$

Finally

$$
x: z \mapsto \bar{z}
$$

denotes complex conjugation of $\mathbf{C}$. For any subset $\boldsymbol{S}$ of $\mathbf{C}$ we write also $\overline{\boldsymbol{S}}$ instead of $\varkappa(\boldsymbol{S})$. For any matrix $M$ we write $\bar{M}$ for the complex conjugate of $M$.

We collect in the following lemma a number of simple relations between the matrices, maps and regions introduced above. Here and further on we use the convention that the index $j$ ranges through $\{1,2,3\}$, and accordingly $j+1$ has to be reduced modulo 3 . 


\section{LEMMA 1.1.}
(i) $S^{3}=I$,
(ii) $V_{j+1}=S V_{j} S^{-1}, E_{j+1}=S E_{f} S^{-1}, C=S C S^{-1}$,
(iii) $\operatorname{det} V_{j}=1, \operatorname{det} E_{j}=i, \operatorname{det} C=-i$,
(iv) $V_{j}^{-1}=\bar{V}_{j}, E_{j}^{-1}=\bar{E}_{j}, C^{-1}=-\bar{C}$,
(v) $v_{j}^{-1}=x \circ v_{j} \circ x, e_{j}^{-1}=x \circ e_{j} \circ x, c^{-1}=x \circ c \circ x$,
(vi) $\mathfrak{\vartheta}_{j}=v,(\mathcal{J}), \mathcal{E}_{j}=e_{j}\left(\mathcal{J}^{*}\right), \mathcal{C}=c\left(\mathfrak{J}^{*}\right)$,
(vii) $\vartheta_{j}^{*}=v_{j}\left(\mathcal{J}^{*}\right), \mathrm{C}^{*}=c(\mathrm{~J})$,
(viii) $\mathfrak{\vartheta}_{j+1}=s\left(\mathfrak{\vartheta}_{j}\right), \mathcal{E}_{j+1}=s\left(\mathcal{E}_{j}\right), \mathrm{C}=s(\mathrm{C})$,
(ix) $\mathfrak{\vartheta}_{j+1}^{*}=s\left(\mathfrak{\vartheta}_{j}^{*}\right), \mathrm{C}^{*}=s\left(\mathrm{C}^{*}\right)$.

Proof. Obvious by inspection. Notice that $s$ is the non-euclidean rotation to an angle $2 \pi / 3$ around $\frac{1}{2}(1+i \sqrt{3})$ if $\mathcal{J}$ is considered as the non-euclidean plane in the Poincaré model.

A homographic map $m: z \mapsto(a z+b) /(c z+d)$ is called unimodular if $a, b, c, d \in \mathbb{Z}[i]$ (the ring of Gaussian integers), and $\operatorname{det} m=a d-b c \in \mathcal{U}=\{ \pm 1, \pm i\}$. Notice that the determinant of a unimodular map is defined only as an element in the quotient group $\mathcal{U} / \mathcal{U}^{2}$ consisting of the two elements $\{ \pm 1\},\{ \pm i\}$. A unimodular map $m$ is called properly unimodular if $\operatorname{det} m=\{ \pm 1\}$, and improperly unimodular if $\operatorname{det} m=\{ \pm i\}$.

As usual $\xi, \eta \in \mathbf{C}$ are called equivalent if there exists a unimodular map $m$ with $\eta=m(\xi)$. Also $\xi, \eta \in C$ are called properly (improperly) equivalent if there exists a properly (improperly) unimodular map $m$ with $\eta=m(\xi)$. Obviously each equivalence class of complex numbers consists of either one or two proper equivalence classes.

An immediate consequence of these definitions and Lemma 1.1 (iii) (vi) (vii) is that every boundary point occurring in Fig. 1 is properly equivalent to a real number, and that every boundary point occurring in Fig. $1^{*}$ is improperly equivalent to a real number.

\subsection{Farey sets}

Let $G$ denote the group of all unimodular maps. To any $m \in G$ we associate a Farey set $F(m)$ as follows,

(1) if $m$ is properly unimodular, we define

$$
F(m)=\left\{\begin{array}{l}
m(\mathfrak{J}) \\
m(\overline{\mathfrak{J}})
\end{array},\right.
$$


where we make the unique choice between the two sets $m(\mathcal{J}), m(\bar{J})$ such that $F(m)$ becomes either a circular dise or a halfplane of the form

$$
\left\{z=x+i y \mid y \geqslant b_{0}\right\}, b_{0} \in \mathbf{Z} ;
$$

(2) if $m$ is improperly unimodular, we define

$$
F(m)=\left\{\begin{array}{l}
m\left(\mathfrak{J}^{*}\right) \\
m\left(\overline{\mathfrak{J}^{*}}\right)
\end{array},\right.
$$

where the upper possibility is chosen if $m(\mathfrak{J})$ is a circular disc or a halfplane of the form

or

$$
\left\{z=x+i y \mid x \leqslant a_{0}\right\}, a_{0}=0,-1,-2, \ldots
$$

$$
\left\{z=x+i y \mid x \geqslant a_{0}\right\}, a_{0}=1,2,3, \ldots,
$$

while the lower possibility is chosen if $m(\overline{\mathcal{J}})$ is a circular disc or a halfplane of the form (1.2) or (1.3).

It is easily shown that by (1),(2), the Farey set $F(m)$ is well-defined for all $m \in G$, and we say that $F(m)$ is of circular (triangular) type, respectively. Notice that in Fig. 1 the sets $\mathcal{J}, \mathfrak{V}_{j}$ are Farey sets of circular type, while the sets $\mathcal{E}_{j}, \mathcal{C}$ are Farey sets of triangular type.

Let $\mathcal{F}$ be the set of all Farey sets, i.e.

$$
\mathfrak{F}=\{F(m) \mid m \in G\} \text {. }
$$

A consequence of a previous remark about the boundary points of Fig. 1, Fig. $1^{*}$ is that

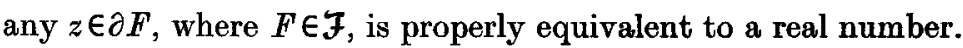

For any $F \in \mathcal{F}$, say $F=F(m)$, we define

$$
\varrho(F)=\left((N(c)+N(d)+N(c+d))^{2}-2\left(N^{2}(c)+N^{2}(d)+N^{2}(c+d)\right)\right)^{1 / 2},
$$

where $m: z \mapsto(a z+b) /(c z+d)$ and $N$ is the Gaussian norm. Apparently $\varrho(F)$ depends not only upon $F$ but also upon $m$. It is a matter of simple trigonometry however, to show that $1 / \varrho(F)$ is the radius of the circle $m(\mathbf{R})$, which is the circumscribed circle of $F$ (notice that $\varrho(F)=0 \Leftrightarrow m(\mathbf{R})$ is a line). We shall prove later in this chapter that

$$
\varrho(F) \in 2 \mathrm{~N}_{0}=\{0,2,4, \ldots\} \text { for all } F \in \mathcal{F} .
$$

The following lemma describes an important relation between Farey sets and Farey triangles (cf. [33] for the definition of a Farey triangle $F T$ and $N(F T)$ ).

LEMMA 1.2. For any $F \in \mathcal{F}$, where $F$ is assumed to be of circular type though not a half plane (i.e. $F$ is a circular disc), either

(i) there is precisely one acute angled Farey triangle $F T_{0}($ say) inscribed in $F$, or

(ii) there is no acute angled Farey triangle inscribed in F, but in return precisely two right 
angled Farey triangles $F T_{1}, F T_{2}$ (say) both inseribed in $F$; the Farey triangles $F T_{1}$ and $F T_{2}$ are congruent, in particular $N\left(F T_{1}\right)=N\left(F T_{2}\right)$.

Proof. Let $F=F(m)$ (say), and assume without restriction that $F=m(\mathcal{J})$; otherwise we could replace the map $m: z \mapsto(a z+b) /(c z+d)$ by $z \mapsto(b z+a) /(d z+c)$. The assumption means that the points $a / c, b / d,(a+b) /(c+d)$ define a positive cyclic ordering of $\partial F$. Equivalently the assumption means that $-d / c \in \mathbf{C} \backslash \mathfrak{Y}$.

Since $F T(a / c, b / d,(a+b) /(c+d))$ is a Farey triangle inscribed in $F$, it follows that an arbitrary Farey triangle $F T=F T\left(p_{1} / q_{1}, p_{2} / q_{2},\left(p_{1}+p_{2}\right) /\left(q_{1}+q_{2}\right)\right)$ inscribed in $F$ with $p_{1} / q_{1}$, $p_{2} / q_{2},\left(p_{1}+p_{2}\right) /\left(q_{1}+q_{2}\right)$ defining a positive cyclic ordering of $\partial F$ is given by

$$
\left(\begin{array}{ll}
p_{1} & p_{2} \\
q_{1} & q_{2}
\end{array}\right)=\left(\begin{array}{ll}
a & b \\
c & d
\end{array}\right)\left(\begin{array}{ll}
r_{1} & r_{2} \\
s_{1} & s_{2}
\end{array}\right)
$$

where $r_{1}, r_{2}, s_{1}, s_{2} \in \mathbf{Z}, r_{1} s_{2}-r_{2} s_{1}=1$.

Consequently

$$
-\frac{q_{2}}{q_{1}}=\frac{s_{2}\left(-\frac{d}{c}\right)-r_{2}}{-s_{1}\left(-\frac{d}{c}\right)+r_{1}}
$$

whence $-q_{2} / q_{1} \in \mathbf{C} \backslash \mathcal{J}$, since $-d / c \in \mathbf{C} \backslash \mathcal{J}$ and $s_{2} r_{1}-s_{1} r_{2}=1$.

Since $F T$ is similar to the triangle with vertices $0,1,-q_{2} / q_{1}$, it follows that $F T$ has all its angles $\leqslant \pi / 2$ if and only if $-q_{2} / q_{1} \in \overline{J^{*}} \backslash\{0,1, \infty\}$.

However, the set $\bar{J}^{*} \backslash\{0,1, \infty\}$ consists of three copies of the fundamental domain (six copies of its boundary) of the modular group $\Gamma$ consisting of all maps $p: z \mapsto\left(r_{1} z+r_{2}\right) /$ $\left(s_{1} z+s_{2}\right)$, where $r_{1}, r_{2}, s_{1}, s_{2} \in \mathbf{Z}, r_{1} s_{2}-r_{2} s_{1}=1$, and operating on the set $\mathbf{C} \backslash \mathcal{J}$. Since each $F T$ inscribed in $F$ occurs for three different $\varphi$ 's, corresponding to the cyclic permutations of the three vertices of $F T$, the result follows readily.

For any Farey set $F$ we define its norm $N(F)$, as follows,

(1a) if $F$ is a halfplane of the form (1.1), we put $N(F)=2$;

(1b) if $F$ is a circular disc, we put $N(F)=N\left(F T_{0}\right)$ or $N(F)=N\left(F T_{1}\right)=N\left(F T_{2}\right)$, depending on whether case (i) or case (ii) of Lemma 1.2 occurs;

(2a) if $F=F(m)$ (say) is of triangular type, and $m(\mathfrak{J})$ is a halfplane of either of the forms (1.2), (1.3), we put $N(F)=2$;

(2b) if $F$ is of triangular type, but not of the form (2a), the three vertices of $F$ are the vertices of a Farey triangle $F T$, and we put $N(F)=N(F T)$. 
Example. $N(\mathcal{J})=N\left(\mathfrak{V}_{1}\right)=N\left(\mathcal{E}_{2}\right)=N\left(\mathcal{E}_{3}\right)=2, N\left(\mathfrak{V}_{2}\right)=N\left(\mathfrak{\vartheta}_{3}\right)=N\left(\mathcal{E}_{1}\right)=N(\mathcal{C})=4$.

\subsection{A generating procedure of Farey sets}

We consider the set

$$
\mathfrak{F}_{n}=\left\{F\left(m_{n}\right) \mid M_{n}=T_{0} T_{1} \ldots T_{n}\right\}, n \in \mathbf{N}_{0},
$$

where

$$
\begin{aligned}
& T_{0}=V_{1}^{b_{0}}, b_{0} \in \mathbf{Z} ; T_{1} \neq V_{1}, \\
& T_{\nu} \in\left\{V_{j}, E_{j}, C\right\} \quad \text { if } \operatorname{det} T_{0} T_{1} \ldots T_{\nu-1}= \pm 1 \\
& T_{\nu} \in\left\{V_{j}, C\right\} \quad \text { if } \operatorname{det} T_{0} T_{1} \ldots T_{\nu-1}= \pm i
\end{aligned}
$$

for $1 \leqslant \nu \leqslant n$.

Thus $\mathcal{F}_{0}$ is the set of all halfplanes (1.1), and $\mathcal{F}_{1}$ consists of $\mathfrak{\vartheta}_{2}, \mathfrak{\vartheta}_{3}, \mathcal{E}_{1}, \mathcal{E}_{2}, \mathcal{E}_{3}, C$ and all their translates by $b_{0} i, b_{0} \in \mathbf{Z}$.

It follows easily by induction on $n$ that

$$
\begin{aligned}
& F\left(m_{n}\right)=m_{n}(\mathcal{Y}) \quad \text { if } \operatorname{det} m_{n}=\{ \pm 1\}, \\
& F\left(m_{n}\right)=m_{n}\left(\mathfrak{Y}^{*}\right) \quad \text { if } \operatorname{det} m_{n}=\{ \pm i\} .
\end{aligned}
$$

Also every Farey set of circular type $F\left(m_{n}\right)=m_{n}(\mathcal{J}) \in \mathcal{F}_{n}$ is divided into seven Farey sets in $\mathfrak{F}_{n+1}$ (cf. Fig. 2), three of which are of circular type, namely $F\left(m_{n} \circ v_{j}\right)=\left(m_{n} \circ v_{j}\right)(\mathcal{J})=$ $m_{n}\left(\mathfrak{\vartheta}_{j}\right)$, while four are of triangular type, namely $F\left(m_{n} \circ e_{j}\right)=\left(m_{n} \circ e_{j}\right)\left(\mathcal{J}^{*}\right)=m_{n}\left(\mathcal{E}_{j}\right)$ and $F\left(m_{n} \circ c\right)=\left(m_{n} \circ c\right)\left(\mathcal{Y}^{*}\right)=m_{n}(\mathcal{C})$. Similarly every Farey set] of triangular type $F\left(m_{n}\right)=$ $m_{n}\left(\mathfrak{J}^{*}\right) \in \mathcal{F}_{n}$ is divided into four Farey sets in $\mathcal{F}_{n+1}$ (ef. Fig. $2^{*}$ ), three of which are of triangular type, namely $F\left(m_{n} \circ v_{j}\right)=\left(m_{n} \circ v_{j}\right)\left(\mathcal{J}^{*}\right)=m_{n}\left(\mho_{j}^{*}\right)$, while one is of circular type, namely $F\left(m_{n} \circ c\right)=\left(m_{n} \circ c\right)(\mathcal{J})=m_{n}\left(\mathcal{C}^{*}\right)$. Of course Fig. 1 is a special case of Fig. 2; however Fig. $1^{*}$, though similar to Fig. $2^{*}$, is not a special case of Fig. $2^{*}$, since $\mathfrak{Z}^{*}$ is not a Farey set.

It follows easily by induction that each parallel strip $\left\{z=x+i y \mid b_{0} \leqslant y \leqslant b_{0}+1\right\}, b_{0} \in \mathbf{Z}$, is tessellated into Farey sets from $\mathfrak{F}_{n}, n \geqslant 1$, and indeed into $2 \cdot 5^{n-1}$ Farey sets of cireular type, and $4 \cdot 5^{n-1}$ Farey sets of triangular type.

In Fig. 2, Fig. $2^{*}$, the Farey set $F\left(m_{n} \circ v_{j}\right)$ lies at vertex number $j$, and $F\left(m_{n} \circ e_{j}\right)$ lies at edge number $j$, while $F\left(m_{n} \circ c\right)$ lies centrally; this explains the use of the symbols $V_{j}, E_{j}, C$.

Lemia 1.3. Let $F\left(m_{n}\right)$ be an arbitrary Farey set in $\mathcal{F}_{n}, n \geqslant 0$, and put (cf. the short-hand notation in Fig. 2, Fig. $2^{*}$, where the points $p_{j}\left(q_{j}, p_{j}^{\prime} / q_{j}^{\prime}\right.$ are represented simply by $\left.j, j^{\prime}\right)$ 


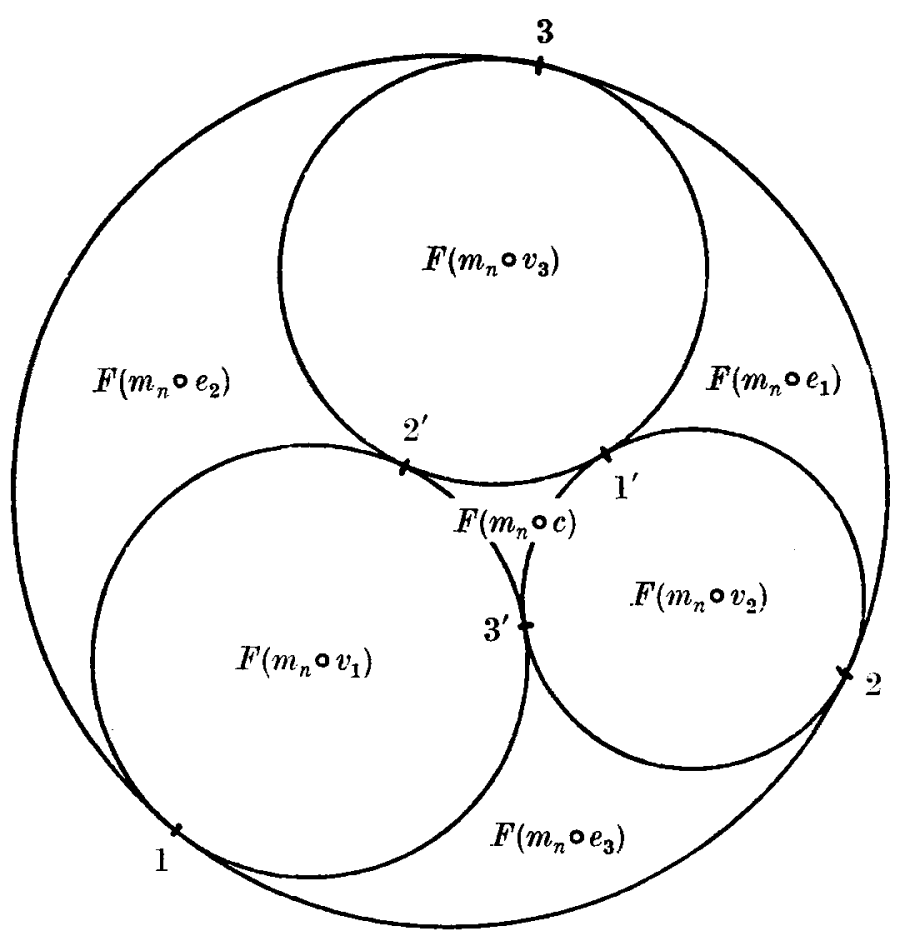

Fig. 2

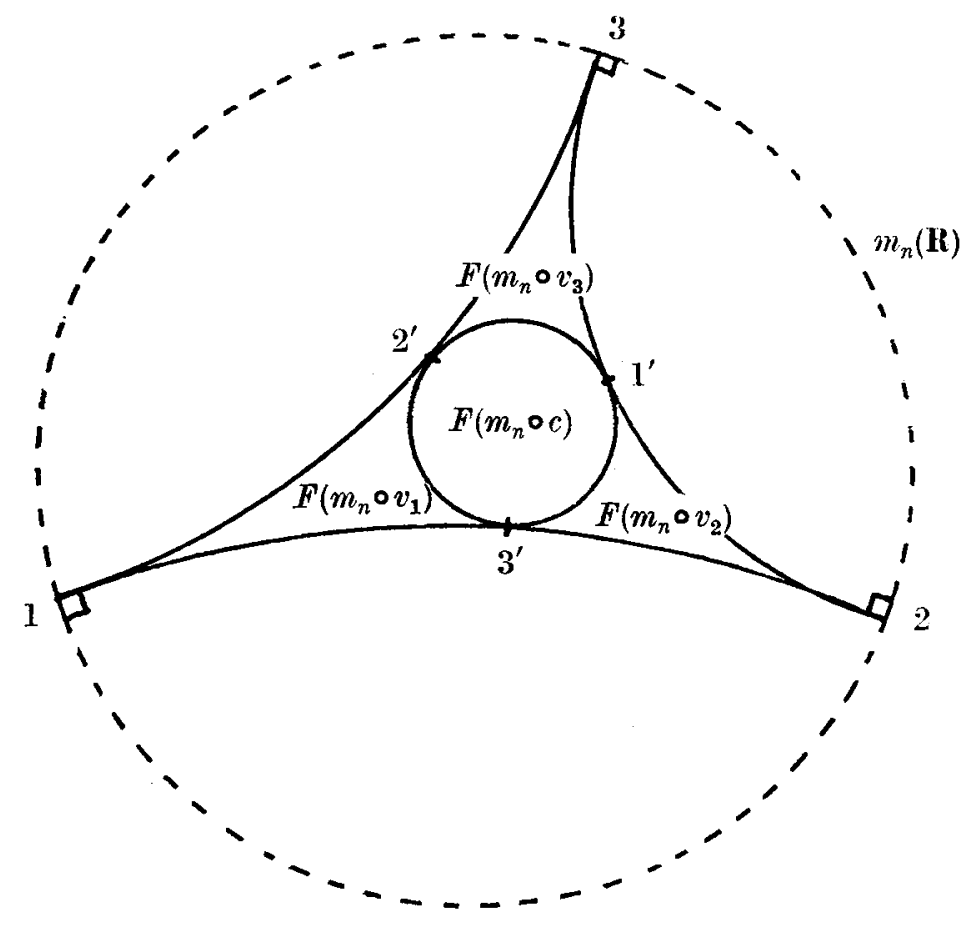

Fig. 2* 


$$
\begin{aligned}
\left(\begin{array}{lll}
p_{1} & p_{2} & p_{3} \\
q_{1} & q_{2} & q_{3}
\end{array}\right) & =M_{n}\left(\begin{array}{lll}
1 & 0 & 1 \\
0 & 1 & 1
\end{array}\right) \\
\left(\begin{array}{lll}
p_{1}^{\prime} & p_{2}^{\prime} & p_{3}^{\prime} \\
q_{1}^{\prime} & q_{2}^{\prime} & q_{3}^{\prime}
\end{array}\right) & =M_{n} C\left(\begin{array}{lll}
1 & 0 & 1 \\
0 & 1 & 1
\end{array}\right) .
\end{aligned}
$$

Also put

$$
\begin{aligned}
& N_{j}=N\left(q_{j}\right), N_{j}^{\prime}=N\left(q_{j}^{\prime}\right), N=N_{1}+N_{2}+N_{3}, \\
& N^{\prime}=N_{1}^{\prime}+N_{2}^{\prime}+N_{3}^{\prime}, N^{(2)}=N_{1}^{2}+N_{2}^{2}+N_{3}^{2} .
\end{aligned}
$$

(i) In the subdivisions of $F\left(m_{n}\right)$ the following norm relations are valid,

$$
\begin{aligned}
& N_{1}+N_{1}^{\prime}=N_{2}+N_{2}^{\prime}=N_{3}+N_{3}^{\prime}, \\
& N^{\prime}=2 N+3\left(N^{2}-2 N^{(2)}\right)^{1 / 2} .
\end{aligned}
$$

(ii) If det $m_{n}=\{ \pm 1\}, n \geqslant 1$, then $F T\left(p_{1} / q_{1}, p_{2} / q_{2}, p_{3} / q_{3}\right)$ is acute angled or right angled.

(iii) $N\left(F\left(m_{n}\right)\right)=N=N\left(F T\left(p_{1} / q_{1}, p_{2} / q_{2}, p_{3} / q_{3}\right)\right)$.

(iv) If det $m_{n}=\{ \pm 1\}$, then (cf. Fig. 2)

$$
\min \left(N\left(F\left(m_{n} \circ v_{j}\right)\right), N\left(F\left(m_{n} \circ e_{j}\right)\right), N\left(F\left(m_{n} \circ c\right)\right)\right) \geqslant N\left(F\left(m_{n}\right)\right),
$$

with equality if and only if $N\left(F\left(m_{n}\right)\right)=2$.

(iv*) If det $m_{n}=\{ \pm i\}$, then (cf. Fig. $2^{*}$ )

$$
\min \left(N\left(F\left(m_{n} \circ v_{j}\right)\right), N\left(F\left(m_{n} \circ c\right)\right)\right) \geqslant N\left(F\left(m_{n}\right)\right),
$$

with equality if and only if $N\left(F\left(m_{n}\right)\right)=2$.

(v)

$$
\mathcal{I}=\bigcup_{n=0}^{\infty} \mathcal{I}_{n}
$$

where the union is a disjoint one.

Proof. The norm relations of (i) are simply the norm relations (36), (65) of [33].

We prove (ii) by induction on $n$. If $\operatorname{det} m_{1}=\{ \pm 1\}$ we must have $M_{1}=V_{1}^{b_{0}} V_{2}$ or $M_{1}=V_{1}^{b_{*}} V_{3}$; it follows easily that $F T\left(p_{1} / q_{1}, p_{2} / q_{2}, p_{3} / q_{3}\right)$ is right angled. If det $m_{n+1}=$ $\{ \pm 1\}$, either

(a) det $m_{n}=\{ \pm 1\}$ and $m_{n+1}=m_{n} \circ v_{j}$ (cf. Fig. 2),

or

(b) $\operatorname{det} m_{n}=\{ \pm i\}$ and $m_{n+1}=m_{n} \circ c$ (cf. Fig. $2^{*}$ ).

Case (a). Suppose without restriction that

$$
N_{1} \leqslant N_{2} \leqslant N_{3} \leqslant N_{1}+N_{2},
$$


the last inequality being the inductive assumption. Then by the norm relations of (i),

$$
\begin{gathered}
N_{1}^{\prime} \geqslant N_{2}^{\prime} \geqslant N_{3}^{\prime}, \\
N_{3}^{\prime}=N+\left(N^{2}-2 N^{(2)}\right)^{\frac{1}{2}}-N_{3} \geqslant N-N_{3}=N_{1}+N_{2} \geqslant N_{3}, \\
N_{2}^{\prime} \leqslant N_{3}^{\prime}+N_{1}, N_{1}^{\prime} \leqslant N_{3}^{\prime}+N_{2}, N_{1}^{\prime} \leqslant N_{2}^{\prime}+N_{3} ;
\end{gathered}
$$

however this proves the assertion (cf. Fig. 2).

Case (b). Suppose without restriction that $N_{1}^{\prime}=\max N_{3}^{\prime}$. Then by the norm relations of (i),

$$
\begin{aligned}
N_{1}^{\prime} & =N+\left(N^{2}-2 N^{(2)}\right)^{1 / 2}-N_{1} \leqslant N+2\left(N^{2}-2 N^{(2)}\right)^{1 / 2}+N_{1} \\
& =\left(N+\left(N^{2}-2 N^{(2)}\right)^{1 / 2}-N_{2}\right)+\left(N+\left(N^{2}-2 N^{(2)}\right)^{1 / 2}-N_{3}\right)=N_{2}^{\prime}+N_{3}^{\prime},
\end{aligned}
$$

thus proving the inductive step.

The truth of (iii) follows now immediately from the very definition of $N\left(F\left(m_{n}\right)\right)$, (1.4), (1.5) and (ii).

(iv) and (iv*) are proved by straightforward applications of the norm relations of (i).

Finally, (v), which essentially tells that any $F \in \mathcal{F}$ is contained in some $\mathcal{F}_{n}, n \geqslant 0$, is proved by an easy induction on $N(F)$.

In view of the disjoint decomposition in Lemma $1.3(\mathrm{v})$ any $F \in \mathcal{F}$ lies in a unique $\mathcal{I}_{n}, n \geqslant 0$. We call $n$ the order of $F$ and write accordingly $n=\operatorname{ord} F$.

\section{LEMMA 1.4. Let $F \in \varsubsetneqq$ be an arbitrary Farey set. Then}

(i) $N(F) \in\{2,4,6, \ldots\}=2 \mathrm{~N}$,

(ii) $\varrho(F) \in\{0,2,4, \ldots\}=2 \mathbf{N}_{0}$,

(iii) $\operatorname{diam} F \leqslant 4(N(F))^{-1 / 2}$ if $N(F)>2$.

Proof. Properties (i), (ii) are proved simultaneously by induction on ord $F$, starting with ord $F=0$, where $N(F)=2$ and $\varrho(F)=0$. Assume for the inductive step that $F=F\left(m_{n}\right) \in \mathcal{F}_{n}$ satisfies conditions (i), (ii), then we have to show the validity of (i), (ii) also for each of the seven (or four) Farey sets $F\left(m_{n} \circ t_{n+1}\right)$ in the subdivision of $F=F\left(m_{n}\right)$.

With the notation of Lemma 1.3 we know that $N(F)=N \equiv 0(\bmod 2), \varrho(F)=$ $\left(N^{2}-2 N^{(2)}\right)^{1 / 2} \equiv 0(\bmod 2)$ (that $\varrho(F)$ is integral follows from the second norm relation of Lemma 1.3(i)), hence by the norm relations

$$
N_{j}+N_{j}^{\prime}=N+\left(N^{2}-2 N^{(2)}\right)^{1 / 2} \equiv 0(\bmod 2),
$$

which proves the inductive step as regards (i) (cf. Fig. 2, Fig. $2^{*}$ ). The inductive step concerning (ii) follows when applying the second norm relation to $F\left(m_{n} \circ t_{n+1}\right)$. 
In the proof of (iii) we use again the notation of Lemma 1.3 with $F=F\left(m_{n}\right)$ and $F T=F T\left(p_{1} / q_{1}, p_{2} / q_{2}, p_{3} / q_{3}\right)$. In case $\operatorname{det} m_{n}=\{ \pm 1\}$ it follows from Lemma 1.3 (ii) that $\operatorname{diam} F \leqslant 2 / 3^{1 / 2} \operatorname{diam} F T$;

in case $\operatorname{det} m_{n}=\{ \pm i\}$ we have simply (also when $F T$ is obtuse angled)

$$
\operatorname{diam} F=\operatorname{diam} F T \text {. }
$$

In order to estimate diam $F T$ we may assume without restriction that $N_{1} \geqslant N_{2} \geqslant N_{3}$, and then

$$
\begin{aligned}
\operatorname{diam} F T & =\left|p_{2} / q_{2}-p_{3} / q_{3}\right| \leqslant\left|p_{1} / q_{1}-p_{2} / q_{2}\right|+\left|p_{1} / q_{1}-p_{3} / q_{3}\right| \\
& =\left(N_{1} N_{2}\right)^{-1 / 2}+\left(N_{1} N_{3}\right)^{-1 / 2} \leqslant 2 N_{1}^{-1 / 2} \leqslant 2(N / 3)^{-1 / 2}
\end{aligned}
$$

Altogether, this proves inequality (iii).

Finally, I want to point out that the circles and mesh triangles of L. R. Ford [14] are special Farey sets, as described in the following

Lемма 1.5. (i) The circles of Ford are precisely the Farey sets $F\left(m_{n}\right)$, where either $n=0$ and $M_{0}=V_{j}(j=2,3)$ or $n \geqslant 1$ and $M_{n}=E_{j} T_{1} \ldots T_{n-1} C$ with $T_{1}, \ldots, T_{n-1} \in\left\{V_{k}, V_{l}\right\},(j, k, l)$ being any permutation of $(1,2,3)$.

(ii) The mesh triangles of Ford are precisely the Farey sets $F\left(m_{n}\right)$, where $n \geqslant 1$ and $M_{n}=E_{j} T_{1} \ldots T_{n}$ with $T_{1}, . ., T_{n} \in\left\{V_{k}, V_{l}\right\},(j, k, l)$ being any permutation of $(1,2,3)$.

The proof of Lemma 1.5 follows readily by the well-known interrelation between Farey fractions and regular continued fractions.

\subsection{Dual Farey sets}

To any unimodular map $m$ we associate a dual Farey set $F^{*}(m)$ as follows,

(1) if $m$ is properly unimodular, we define

$$
F^{*}(m)= \begin{cases}m\left(\mathcal{J}^{*}\right) & \text { if } F(m)=m(\mathcal{J}) \\ m\left(\overline{\mathfrak{J}}^{*}\right) & \text { if } F(m)=m(\overline{\mathfrak{J}})\end{cases}
$$

(2) if $m$ is improperly unimodular, we define

$$
F^{*}(m)= \begin{cases}m(\mathcal{J}) & \text { if } F^{\prime}(m)=m\left(\mathcal{J}^{*}\right) \\ m(\overline{\mathcal{J}}) & \text { if } F(m)=m\left(\overline{\mathfrak{J}^{*}}\right)\end{cases}
$$


Further we let $\exists^{*}$ denote the set of all dual Farey sets, which are contained in the parallel strip

$$
\{z=x+i y \mid 0 \leqslant x \leqslant 1\} \text {. }
$$

The following result is easily deduced from Lemma $1.3(\nabla)$.

LЕмм A 1.6. The set ' I* can be described as

$$
I^{*}=\bigcup_{n=0}^{\infty} \mathcal{I}_{n}^{*}
$$

where the union is a disjoint one, and

$$
\mathcal{F}_{n}^{*}=\left\{F^{*}\left(m_{n}\right) \mid M_{n}=T_{0} T_{1} \ldots T_{n}\right\}, n \geqslant 0,
$$

where

$$
\begin{aligned}
& T_{0}=V_{1}^{b_{0}}, b_{0} \in Z ; T_{1} \neq V_{1} \\
& T_{\nu} \in\left\{V_{j}, C\right\} \quad \text { if } \operatorname{det} T_{0} T_{1} \ldots T_{\nu-1}= \pm 1, \\
& T_{\nu} \in\left\{V_{j}, E_{j}, C\right\} \quad \text { if } \operatorname{det} T_{0} T_{1} \ldots T_{\nu-1}= \pm i
\end{aligned}
$$

for $1 \leqslant \nu \leqslant n$.

Obviously the full content of sections 1.2 and 1.3 carry over to dual Farey sets. Notice in particular that $\mathfrak{y}^{*}, \mathfrak{\vartheta}_{1}^{*}, \vartheta_{2}^{*}, \vartheta_{3}^{*}$ are dual Farey sets of triangular type, while $C^{*}$ is a dual Farey set of circular type.

\section{Chapter 2}

\section{Regular and dually regular chains}

\subsection{Representation of complex numbers}

DEFINITION 2.1. A regular chain is an infinite product

$$
T_{0} T_{1} \ldots T_{n} \ldots
$$

where

$$
T_{0}=V_{1}^{b_{0}}, b_{0} \in \mathbf{Z} ; T_{1} \neq V_{1}
$$

and

$$
\begin{aligned}
& T_{n} \in\left\{V_{j}, E_{j}, C\right\} \quad \text { if } \operatorname{det} T_{0} T_{1} \ldots T_{n-1}= \pm 1 \\
& T_{n} \in\left\{V_{j}, C\right\} \quad \text { if } \operatorname{det} T_{0} T_{1} \ldots T_{n-1}= \pm i
\end{aligned}
$$

for $n \geqslant 1$, with the additional restriction that no $n_{0} \in \mathbf{N}, j \in\{1,2,3\}$, exist, such that $T_{n}=V_{j}$ for all $n \geqslant n_{0}$. 
DEFINITION 2.1*. A dually regular chain is an infinite product

$$
T_{0} T_{1} \ldots T_{n} \ldots
$$

where

$$
T_{0}=V_{1}^{b_{0}}, b_{0} \in \mathbf{Z} ; T_{1} \neq V_{1}
$$

and

$$
\begin{aligned}
& T_{n} \in\left\{V_{j}, C\right\} \quad \text { if } \operatorname{det} T_{0} T_{1} \ldots T_{n-1}= \pm 1 \\
& T_{n} \in\left\{V_{j}, E_{j}, C\right\} \quad \text { if } \operatorname{det} T_{0} T_{1} \ldots T_{n-1}= \pm i
\end{aligned}
$$

for $n \geqslant 1$, with the additional restriction that no $n_{0} \in \mathbf{N}, j \in\{1,2,3\}$, exist, such that $T_{n}=V$, for all $n \geqslant n_{0}$.

In connection with these definitions we introduce some standard notation (valid in both cases),

$$
\begin{gathered}
M_{n}=T_{0} T_{1} \ldots T_{n} \\
\left(\begin{array}{lll}
p_{1}^{(n)} & p_{2}^{(n)} & p_{3}^{(n)} \\
q_{1}^{(n)} & q_{2}^{(n)} & q_{3}^{(n)}
\end{array}\right)=M_{n}\left(\begin{array}{lll}
1 & 0 & 1 \\
0 & 1 & 1
\end{array}\right), \\
m_{n}: z \mapsto \frac{p_{1}^{(n)} z+p_{2}^{(n)}}{q_{1}^{(n)} z+q_{2}^{(n)}} .
\end{gathered}
$$

For a regular chain we put $F_{n}=F\left(m_{n}\right)$, and we call the sequence $F_{n}, n \in \mathbf{N}_{0}$, a regular chain of Farey sets.

For a dually regular chain we put $F_{n}^{*}=F^{*}\left(m_{n}\right)$, and we call the sequence $F_{n}^{*}, n \in \mathbf{N}_{0}$, a dually regular chain of dual Farey sets.

THEOREM 2.1. For any regular chain

$$
T_{0} T_{1} \ldots T_{n} \ldots
$$

we have

$$
F_{0} \supset F_{1} \supset \ldots \supset F_{n} \supset \ldots
$$

and

$$
\bigcap_{n=0}^{\infty} F_{n}=\{\xi\}
$$

where $\xi \in \mathbf{C} \backslash \mathbf{Q}(i)$. Further

$$
\lim _{n \rightarrow \infty} p_{j}^{(n)} / q_{j}^{(n)}=\xi \quad \text { for } j=1,2,3
$$

THEOR EM 2.1*. For any dually regular chain

$$
T_{0} T_{1} \ldots T_{n} \ldots
$$


we have

$$
F_{0}^{*} \supset F_{1}^{*} \supset \ldots \supset F_{n}^{*} \supset \ldots
$$

and

$$
\bigcap_{n=0}^{\infty} F_{n}^{*}=\{\xi\}
$$

where $\xi \in\{z=x+i y \mid 0 \leqslant x \leqslant 1\} \backslash \mathbf{Q}(i)$. Further

$$
\lim _{n \rightarrow \infty} p_{j}^{(n)} / q_{j}^{(n)}=\xi \quad \text { for } j=1,2,3 .
$$

Proof. From section 1.3 follows immediately that the sequence $F_{n}, n \geqslant 0$, is a decreasing sequence of Farey sets, and since each $F_{n}$ is a compact set on the Riemann sphere. it follows that

$$
\bigcap_{n=0}^{\infty} F_{n} \neq \varnothing
$$

By definition a regular chain is not of the form

$$
V_{1}^{b_{\bullet}} E_{k} \vec{V}_{1},(k=2,3),
$$

where the arrow denotes periodicity. Let $n_{0} \in \mathrm{N}$ be the smallest integer such that

$$
T_{0} T_{1} \ldots T_{n_{\bullet}} \neq V_{1}^{b_{0}} E_{k} V_{1}^{n_{0}-1},(k=2,3),
$$

then clearly

$$
N\left(F_{0}\right)=N\left(F_{1}\right)=\ldots=N\left(F_{n_{0}-1}\right)=2<N\left(F_{n_{0}}\right)=4 .
$$

Using Lemma 1.3 (iv) (iv*) we find that

$$
4=N\left(F_{n_{0}}\right)<N\left(F_{n_{0}+1}\right)<\ldots,
$$

and hence by Lemma 1.4 (i) (iii)

$$
\lim _{n \rightarrow \infty} \operatorname{diam} \boldsymbol{F}_{n}=\mathbf{0} \text {. }
$$

Altogether this proves Theorem 2.1, except for the assertion that $\xi \in \mathbf{C} \backslash \mathbf{Q}(i)$. Notice that $q_{j}^{(n)} \neq 0$ for $n \geqslant n_{0}$.

It is a simple geometric fact (cf. [33], Lemma 3), using Lemma 1.3 (ii) in case $\operatorname{det} m_{n}=\{ \pm 1\}$, that

$$
F_{n} \subset \bigcup_{j=1}^{3} B\left(p_{j}^{(n)} / q_{j}^{(n)} ;\left(\sqrt{2}\left|q_{j}^{(n)}\right|^{2}\right)^{-1}\right), \quad n \geqslant n_{0}
$$

where

$$
B\left(z_{0} ; r\right)=\left\{z \in \mathbf{C}|| z-z_{0} \mid \leqslant r\right\} .
$$

Hence it follows that the inequality

$$
\left|\xi-p_{j}^{(n)} / q_{j}^{(n)}\right| \leqslant\left(\sqrt{2}\left|q_{j}^{(n)}\right|^{2}\right)^{-1}
$$

is satisfied for infinitely many pairs $(j, n), j \in\{1,2,3\}, n \geqslant n_{0}$. 
However, the restriction put on a regular chain not to be periodic with period $V$, clearly has the effect that this inequality has infinitely many different solutions $p_{j}^{(n)} / q_{j}^{(n)}$; hence by a standard argument $\xi \in \mathbf{C} \backslash \mathbf{Q}(i)$.

Theorem 2.1* follows immediately from Theorem 2.1 by the very definition of $F^{*}\left(m_{n}\right)$ in section 1.4 .

In connection with Theorems $2.1,2.1^{*}$ we introduce some further notation.

First of all we say that the regular (dually regular) chain $T_{0} T_{1} \ldots T_{n} \ldots$ represents $\xi$ or converges to $\xi$, and we indicate this in writing

$$
\xi=\left[T_{0} T_{1} \ldots T_{n} \ldots\right] .
$$

Further for any regular (dually regular) chain $T_{0} T_{1} \ldots T_{n} \ldots$ we notice that

$$
T_{n} T_{n+1} T_{n+2} \ldots, n \in \mathrm{N}_{0},
$$

is either a regular chain or a dually regular chain; in either case we put

$$
\xi_{n}=\left[T_{n} T_{n+1} \ldots\right], n \in \mathbf{N}_{0},
$$

which we call the $n^{\prime}$ th complete quotient of the given regular (dually regular) chain. It follows from Theorems 2.1, 2.1*, (1.4), (1.5), Lemma 1.3 (ii) and the proof of Lemma 1.2, that for all $n \in \mathbf{N}_{\mathbf{0}}$,

$$
\begin{gathered}
\xi_{n}=t_{n}\left(\xi_{n+1}\right) \\
\xi_{n+1} \in \mathcal{J},-q_{2}^{(n)} / q_{1}^{(n)} \in \overline{\mathcal{J}^{*}} \text { if } \operatorname{det} M_{n}= \pm 1 \\
\xi_{n+1} \in \mathcal{J}^{*},-q_{2}^{(n)} / q_{1}^{(n)} \in \overline{\mathcal{J}} \text { if } \operatorname{det} M_{n}= \pm i
\end{gathered}
$$

Using (2.2) repeatedly we get

$$
\xi=\xi_{0}=m_{n}\left(\xi_{n+1}\right), n \in \mathbf{N}_{\mathbf{0}},
$$

whence for $n \geqslant n_{0}$ (to secure that $q_{j}^{(n)} \neq 0$ ),

$$
\begin{aligned}
& \xi_{0}-p_{1}^{(n)} / q_{1}^{(n)}=-\varepsilon_{n}\left(q_{1}^{(n)}\left(q_{1}^{(n)} \xi_{n+1}+q_{2}^{(n)}\right)\right)^{-1}, \\
& \xi_{0}-p_{2}^{(n)} / q_{2}^{(n)}=\varepsilon_{n} \xi_{n+1}\left(q_{2}^{(n)}\left(q_{1}^{(n)} \xi_{n+1}+q_{2}^{(n)}\right)\right)^{-1}, \\
& \xi_{0}-p_{3}^{(n)} / q_{3}^{(n)}=\varepsilon_{n}\left(\xi_{n+1}-1\right)\left(q_{3}^{(n)}\left(q_{1}^{(n)} \xi_{n+1}+q_{2}^{(n)}\right)\right)^{-1},
\end{aligned}
$$

where

$$
\varepsilon_{n}=p_{1}^{(n)} q_{2}^{(n)}-p_{2}^{(n)} q_{1}^{(n)} \in\{ \pm 1, \pm i\} .
$$

Taking absolute values we obtain the following approximation formulae (for $n \geqslant n_{0}$ ),

$$
\begin{aligned}
& c_{1}^{(n)}=\left|\xi_{n+1}+q_{2}^{(n)} / q_{1}^{(n)}\right|, \\
& c_{2}^{(n)}=\left|\xi_{n+1}^{-1}+q_{1}^{(n)} / q_{2}^{(n)}\right|, \\
& c_{3}^{(n)}=\left|\left(\xi_{n+1}-1\right)^{-1}+q_{1}^{(n)} / q_{3}^{(n)}\right|,
\end{aligned}
$$

2-752903 Acta mathematica 134. Imprimé le 30 Juillet 1975 
where we have put for abbreviation

$$
c_{j}^{(n)}=\left(\left|q_{j}^{(n)}\right|\left|q_{j}^{(n)} \xi_{0}-p_{j}^{(n)}\right|\right)^{-1} .
$$

THसOR м 2.2. For any $\xi \in C$ not properly equivalent to a real number, there is precisely one regular chain $\mathrm{ch} \xi$ representing $\xi$. For any $\xi \in \mathbf{C} \backslash \mathbf{Q}(i)$, which is properly equivalent to a real number, there are precisely two regular chains $\operatorname{ch}_{1} \xi, \operatorname{ch}_{2} \xi$ representing $\xi$.

THEОВем 2.2*. For any $\xi \in\{z=x+i y \mid 0<x<1\}$ not improperly equivalent to a real number, there is precisely one dually regular chain $\mathrm{ch}^{*} \xi$ representing $\xi$. For any $\xi \in\{z=x+i y \mid$ $0<x<1\}$, which is improperly equivalent to a real number, there are precisely two dually regular chains $\operatorname{ch}_{1}^{*} \xi, \operatorname{ch}_{2}^{*} \xi$ representing $\xi$. For any $\xi \in \mathbf{C} \backslash Q(i)$ with $\operatorname{Re} \xi \in\{0,1\}$ there is precisely one dually regular chain $\mathrm{ch}^{*} \xi$ representing $\xi$.

Proof. In case $\xi \in C$ is not properly equivalent to a real number, and hence $\xi \notin \partial F$ for any $F \in \mathcal{F}$, it follows from the results in section 1.3 that for any $n \in \mathbf{N}_{0}$, there is a unique $F_{n} \in \mathcal{F}_{n}$ with $\xi \in F_{n}$. Put $F_{n}=F\left(t_{0} \circ t_{1} \circ \ldots \circ t_{n}\right), n \in \mathrm{N}_{0}$, then it is obvious that $T_{0} T_{1} \ldots T_{n} \ldots$ is a well-defined regular chain representing $\xi$, and clearly also the only one.

In case $\alpha \in \mathbf{R} \backslash \mathbf{Q}$ it follows from the results in section 1.3 that for any $n \in \mathbf{N}_{0}$, there is a unique $F_{n}^{(1)}$ with $\alpha \in \mathbb{F}_{n}^{(1)} \subseteq \mathcal{J}$, and a unique $F_{n}^{(2)}$ with $\alpha \in F_{n}^{(2)} \subseteq \overline{\mathfrak{J}}$; of course $F_{n}^{(2)}=\chi\left(F_{n}^{(1)}\right)=\overline{F_{n}^{(1)}}$. Put $F_{n}^{(k)}=F\left(t_{0}^{(k)} \circ t_{1}^{(k)} \circ \ldots \circ t_{n}^{(k)}\right)$ for $n \in \mathrm{N}_{0}, k \in\{1,2\}$, then it is obvious that $T_{0}^{(k)} T_{1}^{(k)} \ldots T_{n}^{(k)} \ldots$ $(k=1,2)$ are well-defined regular chains representing $\alpha$, and clearly also the only ones.

Let $\alpha=\left[a_{0}, a_{1}, a_{2}, \ldots\right]$ (regular continued fraction), then it is a simple matter to show that

$$
\begin{aligned}
& \operatorname{ch}_{1} \alpha=E_{3} V_{1}^{-a_{0}-1} V_{2} V_{1}^{a_{1}-1} V_{2}^{a_{2}} V_{1}^{a_{3}} V_{2}^{a_{4}} \ldots \text { if } \alpha<0, \\
& \operatorname{ch}_{1} \alpha=E_{1} V_{2}^{a_{1}-1} V_{3}^{a_{2}} V_{2}^{a_{3}} V_{3}^{a_{4}} \ldots \text { if } 0<\alpha<1, \\
& \operatorname{ch}_{1} \alpha=E_{2} V_{1}^{a_{0}-1} V_{3}^{a_{1}} V_{1}^{a_{2}} V_{3}^{a_{3}} V_{1}^{a_{4}} \ldots \text { if } \alpha>1,
\end{aligned}
$$

and that

$$
\begin{aligned}
& \operatorname{ch}_{2} \alpha=V_{1}^{-1} E_{3} V_{1}^{-a_{0}-1} V_{3} V_{1}^{a_{1}-1} V_{3}^{a_{2}} V_{1}^{a_{3}} V_{3}^{a_{4}} \ldots \text { if } \alpha<0, \\
& \operatorname{ch}_{2} \alpha=V_{1}^{-1} C V_{3}^{a_{1}-1} V_{2}^{a_{2}} V_{3}^{a_{3}} V_{2}^{a_{4}} \ldots \text { if } 0<\alpha<1, \\
& \operatorname{ch}_{2} \alpha=V_{1}^{-1} E_{2} V_{1}^{a_{0}-1} V_{2}^{a_{1}} V_{1}^{a_{3}} V_{2}^{a_{3}} V_{1}^{a_{4}} \ldots \text { if } \alpha>1 .
\end{aligned}
$$

Now let $\xi \in \mathbf{C} \backslash \mathbf{Q}(i)$ be properly equivalent to a real number, say $\xi=m(\eta)$, where $m$ is properly unimodular and $\eta \in \mathbf{R} \backslash \mathbf{Q}$. By Lemma $1.3(v)$ there is a smallest integer $n_{0} \in \mathbf{N}_{\mathbf{0}}$ such that $\xi \in \partial F$ for some $F \in \mathcal{F}_{n_{0}}$. 
In case $n_{0}=0$, then $\xi=\alpha+i b_{0}$ with $\alpha \in \mathbf{R} \backslash \mathbf{Q}, b_{0} \in \mathbf{Z}$, and clearly

$$
\operatorname{ch}_{1} \xi=V_{1}^{b_{0}} \operatorname{ch}_{1} \alpha, \operatorname{ch}_{2} \xi=V_{1}^{b_{0}} \operatorname{ch}_{2} \alpha \text {. }
$$

In case $n_{0} \geqslant 1$, then $F_{n_{0}} \in \mathcal{F}_{n_{0}}$ is uniquely determined by requiring that $\xi \in F_{n_{0}}$, and that $F_{n_{0}}$ be of circular type. Let $F_{n_{0}}=F\left(t_{0} \circ t_{1} \circ \ldots \circ t_{n_{0}}\right)=F\left(m_{n_{0}}\right)$ be its standard representation (cf. section 1.3). Then $\xi=m_{n_{0}}(\alpha)$ with $\alpha \in \mathbf{R} \backslash \mathbf{Q}$, and we put $A_{1}=m_{n_{0}}(] 0,1[), A_{2}=$ $m_{n_{0}}(] 1, \infty[), A_{3}=m_{n_{0}}(]-\infty, 0\left[\right.$ ), and $\operatorname{ch}_{1} \alpha=U_{0} U_{1} U_{2} \ldots$ (given by (2.12), (2.13), (2.14)).

Clearly

$$
\operatorname{ch}_{1} \xi=T_{0} T_{1} \ldots T_{n_{0}} U_{0} U_{1} U_{2} \ldots
$$

is a regular chain representing $\xi$. Also it is easily verified that there is precisely one other regular chain $\mathrm{ch}_{2} \xi$ representing $\xi$, namely

$$
\operatorname{ch}_{2} \xi=T_{0} T_{1} \ldots T_{n_{0}-1} \hat{T}_{n_{0}} \lambda\left(U_{0}\right) \lambda\left(U_{1}\right) \lambda\left(U_{2}\right) \ldots
$$

where $\lambda$ is the permutation

$$
\lambda:\left(\begin{array}{lllllll}
V_{1} & V_{2} & V_{3} & E_{1} & E_{2} & E_{3} & C \\
V_{1} & V_{3} & V_{2} & E_{1} & E_{3} & E_{2} & C
\end{array}\right)
$$

and

$$
\hat{T}_{n_{0}}=\left\{\begin{array}{lll}
V_{k} & \text { if } \xi \in A_{k} & \text { and } T_{n_{0}}=C \\
E_{k} & \text { if } \xi \in A_{k} & \text { and } T_{n_{0}}=V_{j}(j \neq k) . \\
C & \text { if } \xi \in A_{k} & \text { and } T_{n_{0}}=V_{k}
\end{array}\right.
$$

This proves the second part of Theorem 2.2.

The proof of Theorem $2.2^{*}$ is easily derived from Theorem 2.2 .

\subsection{Equivalence}

The relationship between regular (dually regular) chains for two numbers $\xi, \eta \in \mathbf{C} \backslash \mathbf{Q}(i)$ $(\{z=x+i y \mid 0 \leqslant x \leqslant 1\} \backslash \mathbf{Q}(i))$ that are equivalent (cf. section 1.1) is described in the following theorems.

THEOREM 2.3. $\xi$, $\eta \in \mathbf{C} \backslash \mathbf{Q}(i)$ are properly equivalent if and only if $\xi, \eta$ have regular chains of the form

$$
\begin{aligned}
& \operatorname{ch} \xi=T_{0} T_{1} \ldots T_{g} T_{g+1} T_{g+2} \ldots T_{g+n} \ldots \\
& \operatorname{ch} \eta=U_{0} U_{1} \ldots U_{h} U_{h+1} U_{h+2} \ldots U_{h+n} \ldots
\end{aligned}
$$

with

(i) $\operatorname{det} t_{0} \circ t_{1} \circ \ldots \circ t_{g}=\operatorname{det} u_{0} \circ u_{1} \circ \ldots \circ u_{h}$ 
and

(ii) $U_{h+n}=S^{j} T_{g+n} S^{-j}, n \in \mathbf{N}$,

where $j \in\{1,2,3\}$ is a fixed integer.

THEOR EM 2.3* $\xi, \eta \in\{z=x+i y \mid 0<x<1\} \backslash Q(i)$ are properly equivalent if and only if $\xi, \eta$ have dually regular chains of the form

$$
\begin{aligned}
& \operatorname{ch}^{*} \xi=T_{0} T_{1} \ldots T_{o} T_{o+1} T_{g+2} \ldots T_{g+n} \ldots, \\
& \operatorname{ch}^{*} \eta=U_{0} U_{1} \ldots U_{n} U_{h+1} U_{h+2} \ldots U_{h+n} \ldots
\end{aligned}
$$

with conditions (i), (ii) of Theorem 2.3 satisfied.

THEOREM 2.4. $\xi \in C \backslash Q(i), \eta \in\{z=x+i y \mid 0 \leqslant x \leqslant 1\} \backslash Q(i)$ are improperly equivalent if and only if $\xi$, $\eta$ have chains of the form

$$
\begin{aligned}
& \operatorname{ch} \xi=T_{0} T_{1} \ldots T_{\mathrm{g}} T_{g+1} T_{g+2} \ldots T_{g+n} \ldots \\
& \operatorname{ch}^{*} \eta=U_{0} U_{1} \ldots U_{n} U_{n+1} U_{n+2} \ldots U_{n+n} \ldots
\end{aligned}
$$

with

$$
\operatorname{det} t_{0} \circ t_{1} \circ \ldots \circ t_{g}=\{ \pm i\} \operatorname{det} u_{0} \circ u_{1} \circ \ldots \circ u_{h}
$$

and satisfying condition (ii) of Theorem 2.3 .

Proof. We restrict ourselves to Theorem 2.3, since the two other theorems are proved similarly.

Suppose first that $\xi, \eta$ have regular chains satisfying conditions (i), (ii). Then by (2.5)

$$
\begin{aligned}
& \xi=t_{0} \circ t_{1} \circ \ldots \circ t_{g}\left(\xi_{g+1}\right), \\
& \eta=u_{0} \circ u_{1} \circ \ldots \circ u_{h}\left(\eta_{h+1}\right),
\end{aligned}
$$

and by condition (ii),

$$
\eta_{h+1}=s^{j}\left(\xi_{g+1}\right)
$$

Hence

$$
\eta=u_{0} \circ u_{1} \circ \ldots \circ u_{h} \circ s^{j} \circ\left(t_{0} \circ t_{1} \circ \ldots \circ t_{g}\right)^{-1}(\xi)
$$

by condition (i) and since $\operatorname{det} s=\{ \pm 1\}$, this shows that $\xi$, $\eta$ are properly equivalent.

Conversely, suppose that $\xi, \eta \in \mathbf{C} \backslash \mathbf{Q}(i)$ are properly equivalent, say $\eta=m(\xi)$, where $m: z \mapsto(a z+b)(c z+d)^{-1}$ is properly unimodular, and let

$$
\operatorname{ch} \xi=T_{0} T_{1} \ldots T_{n} \ldots
$$

be a regular chain representing $\xi$. Put $F_{n}=F^{\prime}\left(t_{0} \circ t_{1} \circ \ldots \circ t_{n}\right)$, then it follows that $m\left(F_{n}\right)$ is 
a Farey set of the same type as $F_{n}$ and containg $\eta$, provided $-d / c \notin F_{n}$. However, this condition is certainly satisfied for all $n \geqslant n_{0}$ (say), since $-d / c \neq \xi \in \mathbf{C} \backslash \mathbf{Q}(i)$, and $\lim \operatorname{diam} \boldsymbol{F}_{n}=0$.

Case 1 . We assume there exists a $g \geqslant n_{0}$ such that $F_{g}$ (and hence also $m\left(F_{g}\right)$ ) is of triangular type. Let $m\left(F_{g}\right)=F\left(u_{0} \circ u_{1} \circ \ldots \circ u_{h}\right)$ be the representation of section 1.3, then in particular $\operatorname{det} t_{0} \circ t_{1} \circ \ldots \circ t_{g}=\operatorname{det} u_{0} \circ u_{1} \circ \ldots \circ u_{h}(=\{ \pm i\})$. Also we put

$$
\begin{aligned}
& \left(\begin{array}{lll}
p_{1}^{(g)} & p_{2}^{(g)} & p_{3}^{(g)} \\
q_{1}^{(g)} & q_{2}^{(g)} & q_{3}^{(g)}
\end{array}\right)=T_{0} T_{1} \ldots T_{g}\left(\begin{array}{lll}
1 & 0 & 1 \\
0 & 1 & 1
\end{array}\right), \\
& \left(\begin{array}{lll}
P_{1}^{(h)} & P_{2}^{(h)} & P_{3}^{(h)} \\
Q_{1}^{(h)} & Q_{2}^{(h)} & Q_{3}^{(h)}
\end{array}\right)=U_{0} U_{1} \ldots U_{h}\left(\begin{array}{lll}
1 & 0 & 1 \\
0 & 1 & 1
\end{array}\right) .
\end{aligned}
$$

Since $m$ maps $\partial F_{g}$ onto $\partial m\left(F_{g}\right)$ with preservation of orientation, it follows by (1.5) that $m\left(p_{k}^{(g)} / q_{k}^{(g)}\right)=P_{k+j}^{(h)} / Q_{k+j}^{(h)}$

for $k \in\{1,2,3\}$ with some fixed $j \in\{1,2,3\}$; of course $k+j$ has to be reduced modulo 3 . Now define $U_{h+n}, n \in N$, by condition (ii), then clearly $U_{0} U_{1} \ldots U_{n} U_{h+1} U_{h+2} \ldots U_{h+n} \ldots$ is a regular chain representing $\eta$.

Case 2. We assume for all $n \geqslant n_{0}$ that $F_{n}$ (and hence also $m\left(F_{n}\right)$ ) is of circular type, whence in particular $T_{n} \in\left\{V_{j}\right\}$ for all $n>n_{0}$. Let $u_{n}$ be the maximal angle of $F T\left(p_{1}^{(n)} / q_{1}^{(n)}\right.$, $\left.p_{2}^{(n)} / q_{2}^{(n)}, p_{3}^{(n)} / q_{3}^{(n)}\right)$, then $\pi / 3 \leqslant u_{n} \leqslant \pi / 2$ by Lemma 1.3 (ii).

In case $u_{n}$ does not converge to $\pi / 2$ for $n \rightarrow \infty$, we proceed as in case 1 using the conformal mapping property of $m$ together with Lemma 1.2 (i) and Lemma 1.3 (ii).

In case $u_{n}$ converges to $\pi / 2$ for $n \rightarrow \infty$, it is a simple consequence of the norm relations of Lemma 1.3 (i) that there exists a fixed $\left(n_{1}, k\right)$ with $n_{1}>n_{0}, k \in\{1,2,3\}$, such that $T_{n} \in\left\{V_{1}, V_{2}, V_{3}\right\} \backslash\left\{V_{k}\right\}$ for all $n \geqslant n_{1}$. Hence $\xi \in \partial F^{*}\left(t_{0} \circ t_{1} \circ \ldots \circ t_{n_{2}}\right)$, and since $F^{*}\left(t_{0} \circ t_{1} \circ \ldots \circ t_{n_{1}}\right)$ is a dual Farey set of triangular type, the proof is completed as in case 1 .

\subsection{Approximation theorems}

For any $\xi \in \mathbf{C} \backslash \mathbf{Q}(i)(\{z=x+i y \mid 0 \leqslant x \leqslant 1\} \backslash \mathbf{Q}(i))$ we shall call a reduced fraction $p / q$ with $p, q \in \mathbf{Z}[i], q \neq 0$, a convergent (dual convergent) of $\xi$ if $p / q=p_{j}^{(n)} / q_{j}^{(n)}$ for some $n \in \mathbf{N}_{\mathbf{0}}, j \in\{1,2,3\}$ corresponding to any regular (dually regular) chain of $\xi$.

As in [33] we define the approximation constant of $\mathbf{a} \in \mathbf{C} \backslash \mathbf{Q}(i)$ as

$$
C(\xi)=\lim \sup (|q||q \xi-p|)^{-1},
$$

the $\lim$ sup being taken over all $p, q \in \mathbf{Z}[i], q \neq 0$. 
THEОR Е 2.5. Let $\xi \in \mathbf{C} \backslash Q(i)(\{z=x+i y \mid 0 \leqslant x \leqslant 1\} \backslash Q(i))$; then any irreducible fraction $p / q$ with $p, q \in \mathbf{Z}[i], q \neq 0$, and satisfying

$$
\left|\xi-\frac{p}{q}\right| \leqslant \frac{1}{(1+1 / \sqrt{2})|q|^{2}}
$$

is a convergent (dual convergent) of $\xi$.

Proof. The proof of Theorem 3 of [33] applies with some obvious changes.

THEOREM 2.6. Let $\xi \in \mathbf{C} \backslash \mathbf{Q}(i) \quad(\{z=x+i y \mid 0 \leqslant x \leqslant 1\} \backslash \mathbf{Q}(i)$ have the regular (dually regular) chain $T_{0} T_{1} T_{2} \ldots ;$ then (with the notation of section 2.1 )

$$
C(\xi)=\lim \sup \left(\left|q_{j}^{(n)}\right|\left|q_{j}^{(n)} \xi-p_{j}^{(n)}\right|\right)^{-1},
$$

the $\lim$ sup being taken over all $(n, j) \in \mathbf{N}_{0} \times\{1,2,3\}$.

Proof. The result follows readily from Theorem 2.5 , since

$$
1+1 / \sqrt{2}<\sqrt{3} \leqslant C(\xi)
$$

the last inequality being a consequence of a theorem of Ford [13].

TH ЕоR Ем 2.7. Suppose $\xi \in \mathbf{C} \backslash \mathbf{Q}(i)$ is represented by a purely periodic regular (dually regular) chain,

$$
\left.\xi=\overline{T_{0} T_{1} \ldots T_{k-1}}\right]
$$

(cf. chapter 3). Then

$$
C(\xi)=\sqrt{|D|} / \mu
$$

where $D$ and $\mu$ are determined by

$$
\begin{gathered}
\left(\begin{array}{ll}
a & b \\
c & d
\end{array}\right)=T_{0} T_{1} \ldots T_{k-1}, \\
f(X, Y)=c X^{2}+(d-a) X Y-b Y^{2}, \\
D=(d-a)^{2}+4 b c, \\
\mu=\min \left|f\left(p_{j}^{(l)} / q_{j}^{(l)}\right)\right|,
\end{gathered}
$$

the minimum being taken over all $(l, j) \in\{0,1, \ldots, k-1\} \times\{1,2,3\}$.

Proof. Obviously $\xi=(a \xi+b) /(c \xi+d)$ is a root of $f(x, 1)=0$, and hence

$$
f(X, Y)=c(X-\xi Y)\left(X-\xi^{\prime} Y\right)
$$


By the pure periodicity

$$
f\left(p_{j}^{(l+n k)} / q_{j}^{(l+n k)}\right)=(a d-b c)^{2 n} f\left(p_{j}^{(l)} / q_{j}^{(l)}\right)
$$

in particular

$$
\left|f\left(p_{j}^{(l+n k)} / q_{j}^{(l+n k)}\right)\right|=\left|f\left(p_{j}^{(l)} / q_{j}^{(l)}\right)\right|
$$

for $n \in \mathbf{N}_{0}, 0 \leqslant l \leqslant k-1$.

Consequently by (2.21) and (2.22),

$$
\begin{aligned}
\left(\left|q_{j}^{(l+n k)}\right|\left|q_{j}^{(l+n k)} \xi-p_{j}^{(l+n k)}\right|\right\rangle^{-1}=\frac{|c|\left|p_{j}^{(l+n k)} / q_{j}^{(l+n k)}-\xi^{\prime}\right|}{\mid f\left(p_{j}^{(l)} / q_{j}^{(l)} \mid\right.} & \\
& \rightarrow \frac{|c|\left|\xi-\xi^{\prime}\right|}{\left|f\left(p_{j}^{(l)} / q_{j}^{(l)}\right)\right|}=\frac{\sqrt{|D|}}{\left|f\left(p_{j}^{(l)} / q_{j}^{(l)}\right)\right|} \text { for } n \rightarrow \infty .
\end{aligned}
$$

This proves Theorem 2.7 in view of 'Theorem 2.6.

By Theorem 2.7 it is a finite procedure to calculate $C(\xi)$ for any $\xi \in \mathbf{C} \backslash \mathbf{Q}(i)$ having a periodic regular (dually regular) chain. We shall give three examples of this.

Example 2.1. (Ford [13].) $\xi=\frac{1}{2}(1+i \vec{V})$ has the (unique) regular and dually regular chain

$$
\operatorname{ch} \xi=\operatorname{ch}^{*} \xi=\ddot{C}
$$

It follows that

$$
f(X, Y)=(1-i)\left(X^{2}-X Y+Y^{2}\right), D=6 i, \mu=\sqrt{2}
$$

hence

$$
C(\xi)=\sqrt{3}=\sqrt{4-1 / 1^{2}}=1.7320 \ldots
$$

Example 2.2. $\xi=\frac{1}{2}(1+i(2+\sqrt{99}) / 5$ ) has the (unique) regular chain

$$
\operatorname{ch} \xi=\widehat{V_{1}} \bar{E}_{1} \overline{C E_{1}} \overline{V_{1}} \vec{C}
$$

It follows by a simple calculation that

$$
f(X, Y)=2 i\left(5 X^{2}-(5+2 i) X Y+(6+i) Y^{2}\right), D=396, \mu=10,
$$

hence

$$
C(\xi)=\sqrt{4-1 / 5^{2}}=1.9899 \ldots
$$

Example 2.3. (Cassels [3].) $\xi_{0}=(-1+\sqrt{-15-12 i}) /(2+4 i)$, which is a root of the quadratic form

$$
(1+2 i) X^{2}+X Y+(2-i) Y^{2}
$$


has the (unique) regular chain

$$
\operatorname{ch} \xi_{0}=\overparen{C C E_{2} C V_{1} E_{1} V_{1} C E_{3} C V_{2} E_{2} V_{2} C E_{1} C V_{3} E_{3} \vec{V}_{3}}
$$

of period 18. Since $\xi_{0}$ is improperly equivalent to $\xi_{1}$, and hence $C\left(\xi_{0}\right)=C\left(\xi_{1}\right)$, we consider $\xi_{1}$, which has the purely periodic dually regular chain

$$
\operatorname{ch}^{*} \xi_{1}=\overline{C E_{2} C V_{1} E_{1} \nabla_{1} C E_{3} C V_{2} E_{2} V_{2} C E_{1} C V_{3} E_{3} \vec{V}_{3}} \text {. }
$$

The calculation of $C\left(\xi_{1}\right)$ is much facilitated by the observation that in $\operatorname{ch}^{*} \xi_{1}$,

$$
T_{n+6}=S T_{n} S^{-1} \text { for } n \in \mathbf{N}_{0},
$$

by Lemma 1.1 (ii). Hence by a slightly modified version of Theorem 2.7 we get

$$
\begin{gathered}
\left(\begin{array}{cc}
\tilde{a} & \tilde{b} \\
\tilde{c} & \tilde{d}
\end{array}\right)=C E_{2} C V_{1} E_{1} V_{1} S=\left(\begin{array}{cc}
1+9 i & -7-6 i \\
12+3 i & -11+6 i
\end{array}\right), \\
\tilde{f}(X, Y)=(4+i)\left(3 X^{2}-3 X Y+(2+i) Y^{2}\right), \tilde{D}=(4+i)^{2}(-15-12 i), \\
\tilde{\mu}=\min _{1 \leqslant j \leqslant 3,0 \leqslant l \leqslant 6}\left|f\left(p_{j}^{(l)} / q_{j}^{(l)}\right)\right|=\sqrt{17} \cdot \sqrt{5},
\end{gathered}
$$

hence

$$
C\left(\xi_{0}\right)=C\left(\xi_{1}\right)=\sqrt{|\tilde{D}|} \mid \tilde{\mu}=\sqrt{\frac{3}{5} \sqrt{41}}=1.9599 \ldots .
$$

In chapter 6 we shall prove the result that the set of approximation constants $<2$ is

$$
\left\{\sqrt{4-1 / \Lambda^{2}} \mid \Lambda=1,5,29,65, \ldots\right\} \cup\left\{\sqrt{\frac{3}{5} \sqrt{41}}\right\} .
$$

The three examples above thus represent the three lowest approximation constants.

\section{Chapter 3}

\section{Periodic chains}

\subsection{Periodic chains}

A regular (dually regular) chain

$$
T_{0} T_{1} \ldots T_{n} \ldots
$$

is called periodic with period $k \geqslant 1$ if there exists an $h \geqslant-1$ such that

$$
T_{n}=T_{n+k} \text { for all } n \geqslant h+1,
$$

in which case we write the chain as

$$
T_{0} T_{1} \ldots T_{n} \overline{T_{n+1} T_{n+2} \ldots T_{n+k}}
$$


The chain (3.1) is called purely periodic if we may take $h=-1$. (Notice that in order that (say) $V_{1}^{2} \overrightarrow{C V_{1}} \overrightarrow{V_{1}}$ be considered as a purely periodic regular (dually regular) chain, we have to deviate slightly from the notation used so far and thus allowing $T_{1}$ to be $V_{1}$ ).

THEOREM 3.1. A periodic regular chain represents a $\xi \in \mathbf{C} \backslash \mathbf{Q}(i)$ which is quadratic over $\mathbf{Q}(i)$

THEOR E M 3.1*. A periodic dually regular chain represents a $\xi \in\{z=x+i y \mid 0 \leqslant \dot{x} \leqslant 1\} \backslash \mathbf{Q}(i)$ which is quadratic over $\mathbf{Q}(i)$.

Proof. Suppose $\xi$ has the regular (dually regular) chain (3.1), then by (2.2), (2.5)

$$
\begin{gathered}
\xi=\xi_{0}=m_{h}\left(\xi_{h+1}\right), \\
\xi_{h+1}=t_{h+1} \circ t_{h+2} \circ \ldots \circ t_{h+k}\left(\xi_{h+k+1}\right),
\end{gathered}
$$

with $m_{h}$ and $t_{h+1} \circ t_{h+2} \circ \ldots \circ t_{h+k}$ unimodular. Since $\xi_{h+1}=\xi_{h+k+1}$ by the periodicity, the result follows immediately.

THEOREM 3.2. Every $\xi \in \mathbf{C} \backslash \mathbf{Q}(i)$ which is quadratic over $\mathbf{Q}(i)$ has a periodic regular chain (two in case $\xi$ is properly equivalent to a real number).

THEOREM $3.2^{*}$. Every $\xi \in\{z=x+i y \mid 0 \leqslant x \leqslant 1\} \backslash \mathbf{Q}(i)$ which is quadratic over $\mathbf{Q}(i)$ has a periodic dually regular chain (two in case $\xi$ is improperly equivalent to a real number and $\operatorname{Re} \xi \notin\{0,1\})$.

Proof. Let $\xi$ be quadratic over $\mathbf{Q}(i)$; then $\xi$ is a root of a quadratic equation

$$
A z^{2}+B z+C=0
$$

with $A, B, C \in \mathbf{Z}[i]$, and $D=B^{2}-4 A C$ not a square in $\mathbf{Z}[i]$. Hence

$$
\xi=\frac{-B \pm \sqrt{D}}{2 A}
$$

where we fix $\sqrt{D}$ such that arg $\sqrt{D} \in\left[0, \pi\left[\right.\right.$. In any case $\xi=\xi_{0}$ has the form

$$
\xi_{0}=\frac{\sqrt{D}+P_{0}}{Q_{0}}
$$

with $P_{0}, Q_{0} \in \mathbf{Z}[i]$ and $Q_{0} \mid D-P_{0}^{2}$ (in $\left.\mathbf{Z}[i]\right)$, since

$$
D-P_{0}^{2}=D-B^{2}=-4 A C, \quad Q_{0}= \pm 2 A \text {. }
$$

Let

$$
T_{0} T_{1} \ldots T_{n} \ldots
$$

be any regular (dually regular) chain representing $\xi_{0}$. 
As the first step of the proof we want to show that any complete quotient $\xi_{n}$ of the chain above is of the form

$$
\xi_{n}=\frac{\sqrt{D}+P_{n}}{Q_{n}}
$$

with $P_{n}, Q_{n} \in \mathbf{Z}[i]$ and $Q_{n} \mid D-P_{n}^{2}$.

We proceed by induction. Since we know the result to be true for $\xi_{0}$, suppose it true for $\xi_{n}$ and consider $\xi_{n+1}$, which by (2.2) is of the form

$$
\xi_{n+1}=t_{n}^{-1}\left(\xi_{n}\right)
$$

with

$$
T_{n}=\left(\begin{array}{ll}
a & b \\
c & d
\end{array}\right) \in\left\{V_{j}, E_{j}, C\right\}
$$

Hence

$$
\begin{aligned}
& \xi_{n+1}=\frac{-d \xi_{n}+b}{c \xi_{n}-a}=\frac{-d\left(\sqrt{D}+P_{n}\right)}{c\left(\sqrt{D}+P_{n}\right)-}-a Q_{n} \\
&= \frac{(b c-a d) Q_{n} \sqrt{D}-c d\left(D-P_{n}^{2}\right)+Q_{n}\left((-a d-b c) P_{n}+a b Q_{n}\right)}{c^{2}\left(D-P_{n}^{2}\right)+Q_{n}\left(2 a c P_{n}-a^{2} Q_{n}\right)}
\end{aligned}
$$

However, since $b c-a d=\varepsilon$ is a unit in $Z[i]$ and $Q_{n} \mid D-P_{n}^{2}$, we have

$$
\begin{aligned}
& P_{n+1}=\varepsilon^{-1}\left(-c d\left(D-P_{n}^{2}\right) / Q_{n}-(a d+b c) P_{n}+a b Q_{n}\right), \\
& Q_{n+1}=\varepsilon^{-1}\left(c^{2}\left(D-P_{n}^{2}\right) / Q_{n}+2 a c P_{n}-a^{2} Q_{n}\right)
\end{aligned}
$$

both lying in Z[i]. That $Q_{n+1} \mid D-P_{n+1}^{2}$ does not follow from the unimodularity of $T_{n}$ but is easily checked in each of the seven cases $T_{n} \in\left\{V_{j}, E_{j}, C\right\}$, e.g. in case $T_{n}=C$, we find that

$$
\begin{aligned}
& P_{n+1}=(-1+i)\left(D-P_{n}^{2}\right) / Q_{n}-3 P_{n}+(1+i) Q_{n} \\
& Q_{n+1}=-2\left(D-P_{n}^{2}\right) / Q_{n}-(2+2 i) P_{n}+i Q_{n}
\end{aligned}
$$

and hence

$$
D-P_{n+1}^{2}=Q_{n+1}\left(-i\left(D-P_{n}^{2}\right) / Q_{n}+(2-2 i) P_{n}-2 Q_{n}\right) .
$$

This completes the first step of the proof.

We shall use a prime (') to indicate taking conjugates in the field extension $Q(i) \subset$ $\mathbf{Q}(i, \sqrt{D})$; in particular

$$
\xi_{n}^{\prime}=\frac{-\sqrt{D}+P_{n}}{Q_{n}}
$$


and hence

$$
\begin{aligned}
\xi_{n}-\xi_{n}^{\prime} & =\frac{2 \sqrt{D}}{Q_{n}}, \\
\xi_{n}^{-1}-\xi_{n}^{\prime-1} & =\frac{2 Q_{n} \sqrt{D}}{D-P_{n}^{2}}, \\
\left(\xi_{n}-1\right)^{-1}-\left(\xi_{n}^{\prime}-1\right)^{-1} & =\frac{2 Q_{n} \sqrt{D}}{D-\left(P_{n}-Q_{n}\right)^{2}} .
\end{aligned}
$$

As the second step of the proof we want to show that the sequence of complete quotients $\xi_{0}, \xi_{1}, \ldots$ contains only finitely many different numbers, or equivalently that the two sequences of Gaussian integers $P_{n}, Q_{n}(n \geqslant 0)$ are both bounded. By (3.2) and (3.3) this will follow if we prove the existence of a constant $\delta>0$ such that

$$
\left|\xi_{n}-\xi_{n}^{\prime}\right|>\delta,\left|\xi_{n}^{-1}-\xi_{n}^{\prime-1}\right|>\delta \quad \text { for } n \geqslant 0 .
$$

From the formulae (2.6), (2.7), (2.8) we obtain by conjugating and taking absolute values,

$$
\begin{aligned}
\left|\xi_{n+1}^{\prime}+q_{2}^{(n)} / q_{1}^{(n)}\right| & =\left(\left|q_{1}^{(n)}\right|^{2}\left|\xi_{0}^{\prime}-p_{1}^{(n)} / q_{1}^{(n)}\right|\right)^{-1}, \\
\left|\xi_{n-1}^{\prime-1}+q_{1}^{(n)} / q_{2}^{(n)}\right| & =\left(\left|q_{2}^{(n)}\right|^{2}\left|\xi_{0}^{\prime}-p_{2}^{(n)} / q_{2}^{(n)}\right|\right)^{-1}, \\
\left|\left(\xi_{n+1}^{\prime}-1\right)^{-1}+q_{1}^{(n)} / q_{3}^{(n)}\right| & =\left(\left|q_{3}^{(n)}\right|^{2}\left|\xi_{0}^{\prime}-p_{3}^{(n)} / q_{3}^{(n)}\right|\right)^{-1}
\end{aligned}
$$

Since

$$
\left|q_{j}^{(n)}\right| \rightarrow \infty \text { and }\left|\xi_{0}^{\prime}-p_{j}^{(n)} / q_{j}^{(n)}\right| \rightarrow\left|\xi_{0}^{\prime}-\xi_{0}\right| \neq 0 \text { for } n \rightarrow \infty
$$

it follows that the expressions in (3.6), (3.7), (3.8) all tend to zero for $n \rightarrow \infty$; thus to prove the existence of $\delta$ to be used in (3.5) it suffices to prove the existence of a constant $\delta_{1}>0$ such that

$$
\left|\xi_{n+1}+q_{2}^{(n)} / q_{1}^{(n)}\right|>\delta_{1},\left|\xi_{n+1}^{-1}+q_{1}^{(n)} / q_{2}^{(n)}\right|>\delta_{1}
$$

for all $n \geqslant n_{1}$ (say).

However, it follows from (3.2), (3.3), (3.4), since $Q_{n} \mid D-P_{n}^{2}$ and $Q_{n} \mid D-\left(P_{n}-Q_{n}\right)^{2}$, that

$$
\left|\xi_{n}-\xi_{n}^{\prime}\right|,\left|\xi_{n}^{-1}-\xi_{n}^{\prime-1}\right|,\left|\left(\xi_{n}-1\right)^{-1}-\left(\xi_{n}^{\prime}-1\right)^{-1}\right|
$$

are all $\leqslant 2 \sqrt{|D|}$ for $n \geqslant 0$. Hence using once more that the expressions in (3.6), (3.7), (3.8) tend to zero for $n \rightarrow \infty$, we can find a constant $K>2 \sqrt{|\bar{D}|} \geqslant 2$ (e.g. $K=2 \sqrt{|D|}+1$ ), such that

$$
\left|\xi_{n+1}+q_{2}^{(n)}\right| q_{1}^{(n)}|,| \xi_{n+1}^{-1}+q_{1}^{(n)} / q_{2}^{(n)}|,|\left(\xi_{n+1}-1\right)^{-1}+q_{1}^{(n)} / q_{3}^{(n)} \mid
$$

are all $<K$ for all $n \geqslant n_{1}=n_{1}(K)$. 
We will prove that the inequalities (3.9) are valid with $\delta_{1}=1 /(2 K)$ for all $n \geqslant n_{1}=n_{1}(K)$. More explicitly, the first inequality of (3.9) follows from the second and third inequalities of (3.10), while the second inequality of (3.9) follows from the first and third inequalities of (3.10).

In fact, for each $n \in \mathbf{N}_{\mathbf{0}}$, either (2.3) or (2.4) is valid. Now suppose (to prove the first inequality of (3.9), say) that for some $n \geqslant n_{1}$,

$$
\left|\xi_{n+1}+q_{2}^{(n)} / q_{1}^{(n)}\right| \leqslant \frac{1}{2 K}<\frac{1}{4}
$$

then we have to obtain a contradiction.

From $\left(^{*}\right)$ it follows, independently of whether (2.3) or (2.4) is valid, that $\xi_{n+1}$ and $-q_{2}^{(n)} / q_{1}^{(n)}$ are both in the same circular disc $B(0 ; 1 / 2)$ or $B(1 ; 1 / 2)$; by symmetry we may as well assume that $(2.3)$ is valid, and that $\xi_{n+1}$ and $-q_{2}^{(n)} / q_{1}^{(n)}$ are both in $B(0 ; 1 / 2)$. It follows then from $(2.3)$ and $\left({ }^{*}\right)$, that

$$
-q_{2}^{(n)} / q_{1}^{(n)}=r e^{i(-\pi / 2+u)}, 0<r<1 /(\sqrt{2} K), 0 \leqslant u<\pi_{i}^{\prime},
$$

so that

Hence by (2.3)

$$
\left|\operatorname{Im}\left(-q_{1}^{(n)} / q_{2}^{(n)}\right)\right|=r^{-1} \cos u>K
$$

$$
\left|\xi_{n+1}^{-1}+q_{1}^{(n)} / q_{2}^{(n)}\right| \geqslant\left|\operatorname{Im}\left(\xi_{n+1}^{-1}+q_{1}^{(n)} / q_{2}^{(n)}\right)\right| \geqslant\left|\operatorname{Im}\left(-q_{1}^{(n)} / q_{2}^{(n)}\right)\right|>K
$$

contradicting the second inequality of (3.10). This completes the second step of the proof.

The final step of the proof is now an easy one. Since there are only finitely many different numbers in the sequence $\xi_{0}, \xi_{1}, \ldots$, we can pick five different indices $n_{1}, \ldots, n_{5}$ such that $\xi_{n_{1}}=\ldots=\xi_{n_{b}}$. Among these indices we can pick three, say $n_{1}, n_{2}, n_{3}$, such that $\operatorname{det} m_{n_{1}-1}=\operatorname{det} m_{n_{2}-1}=\operatorname{det} m_{n_{\mathrm{a}}-1}(=\{ \pm 1\},\{ \pm i\})$. Then the number $\xi_{n_{1}}=\xi_{n_{z}}=\xi_{n_{3}}$ has

$$
T_{n_{\mathrm{z}}} T_{n_{\mathrm{x}}+1} \ldots, T_{n_{\mathrm{z}}} T_{n_{\mathrm{z}}+1} \ldots, T_{n_{\mathrm{z}}} T_{n_{\mathrm{s}}+1} \ldots
$$

as regular (dually regular) chains. However, since at most two different regular (dually regular) chains can represent the same number, two of the three chains above must be identical. This proves the periodicity.

The proof above of Theorems $3.2,3.2^{*}$ follows very closely the classical proof of Lagrange for the corresponding theorem about the periodicity of regular continued fractions of (real) quadratic irrationals. The most notable deviation lies in the second step, and is in fact inavoidable because of the differences in the notions of reducedness (cf. section 3.2). 
It is easy to give an alternative proof of Theorems $3.2,3.2^{*}$ using the idea of Charves (cf. [27, pp. 71-72]), however, I shall leave the details to the reader.

\subsection{Purely periodic chains}

DEFINITION 3.1. A number $\xi \in \mathbf{C} \backslash Q(i)$, which is quadratic over $\mathbf{Q}(i)$ with conjugate $\xi^{\prime}$, is called reduced if

$$
\xi \in \mathcal{J} \text { and } \xi^{\prime} \in \overline{J^{*}}
$$

and is called dually reduced if

$$
\xi \in \mathcal{J} * \text { and } \xi^{\prime} \in \overline{\mathfrak{J}} \text {. }
$$

THE OR E M 3.3. Every purely periodic regular (dually regular) chain represents a $\boldsymbol{\xi} \in \mathbf{C} \backslash \mathbf{Q}(i)$, which is quadratic over $\mathbf{Q}(i)$ and reduced (dually reduced).

Proof. To be specific let

$$
\overrightarrow{T_{0} T_{1} \ldots T_{k-1}}
$$

be a purely periodic regular chain. By Theorem 3.1 it follows that $\left[\overrightarrow{\left.T_{0} T_{1} \ldots T_{k-1}\right]}=\right.$ $\xi \in \mathbf{C} \backslash \mathbf{Q}(i)$ and is quadratic over $\mathbf{Q}(i)$. By the pure periodicity

$$
\xi=\xi_{n k} \text { for } n=0,1,2, \ldots
$$

whence, since all complete quotients $\xi_{1}, \xi_{2}, \ldots$ are in $\mathcal{J}$ by $(2.3),(2.4)$, it follows at once that $\xi \in \mathcal{J}$.

Further by the pure periodicity

$$
\operatorname{det} m_{2 n k-1}=\left(\operatorname{det} m_{n k-1}\right)^{2}=\{ \pm 1\}, n \in \mathbf{N} \text {, }
$$

and hence by (2.3),

$$
-q_{2}^{(2 n k-1)} / q_{1}^{(2 n k-1)} \in \overline{\mathcal{J}^{*}} \text { for } n \in \mathbf{N} \text {. }
$$

Finally, using that the expressions in (3.6) tend to zero for $n \rightarrow \infty$, we obtain

$$
\xi_{0}^{\prime}=\xi_{2 n k}^{\prime}=\lim _{n \rightarrow \infty}-q_{2}^{(2 n k-1)} / q_{1}^{(2 n k-1)} \in \overline{\mathcal{J}^{*}},
$$

since $\overline{\mathfrak{J}^{*}}$ is a closed set. Thus $\xi$ is reduced.

The dual part of Theorem 3.3 is proved similarly.

THEOREM 3.4. Every $\xi \in \mathbf{C} \backslash \mathbf{Q}(i)$ which is quadratic over $\mathbf{Q}(i)$ and reduced (dually reduced) has a unique purely periodic regular (dually regular) chain.

Proof. As the first step of the proof we want to establish the uniqueness. 
By the description of Farey sets in section 1.3, there is no Farey set $F$ of circular type with $\partial F \cap \mathcal{J} \backslash\{0,1, \infty\} \neq \varnothing, \partial F \cap \overline{J^{*}} \backslash\{0,1, \infty\} \neq \varnothing$. Consequently, a $\xi \in \mathbf{C} \backslash \mathbf{Q}(i)$, quadratic over $\mathbf{Q}(i)$ and reduced, cannot be properly equivalent to a real number, since otherwise there would exist a properly unimodular map $m$ with

$$
\xi, \xi^{\prime} \in m(\mathbf{R})=\partial F(m) \text {. }
$$

Hence in this case the uniqueness follows from Theorem 2.2.

Similarly, the only dual Farey sets $F^{*}$ of circular type with

$$
\partial F^{*} \cap \mathfrak{J}^{*} \backslash\{0,1, \infty\} \neq \varnothing, \partial F^{*} \cap \overline{\mathfrak{J}} \backslash\{0,1, \infty\} \neq \varnothing,
$$

are $F^{*}\left(e_{j}\right), j=1,2,3$. Consequently for any $\xi \in \mathbf{C} \backslash Q(i)$, quadratic over $Q(i)$ and dually reduced, we must have $\xi \in \partial J^{*}$, and hence the uniqueness follows from Theorem $2.2^{*}$, except for

$$
\xi \in\left\{z=x+i y \mid 0<x<1, y=\left(x-x^{2}\right)^{1 / 2}\right\}
$$

These special $\xi$ have two different dually regular chains, one of the form

$$
T_{0} T_{1} T_{2} \ldots, \text { with } T_{n} \in\left\{V_{2}, V_{3}\right\} \text { for } n \geqslant 0,
$$

the other of the form

$$
V_{1}^{-1} C E_{1} U_{3} U_{4} \ldots, \quad \text { with } U_{n} \in\left\{V_{2}, V_{3}\right\} \text { for } n \geqslant 3
$$

Since chains of the form $\left({ }^{* *}\right)$ are never purely periodic, we have shown the uniqueness also in the dually regular case.

Disregarding dually regular chains of the form $\left(^{* *}\right)$, every $\xi \in \mathbf{C} \backslash \mathbf{Q}(i)$, quadratic over $\mathbf{Q}(i)$ and reduced (dually reduced), has a unique regular (dually regular) chain, which by Theorems 3.2, 3.2* is periodic, say of the form (3.1).

As the second step of the proof we want to show that every complete quotient $\xi_{n}$ belonging to the chain (3.1) is also reduced or dually reduced.

To be specific let $\xi$ be reduced and (3.1) be its unique regular chain. Then we claim, that $\xi_{n}(n \geqslant 1)$ is reduced if $\operatorname{det} m_{n-1}=\{ \pm 1\}$, and dually reduced if $\operatorname{det} m_{n-1}=\{ \pm i\}$.

We proceed by induction on $n$, starting with $n=0$ with $m_{-1}$ being the identity map of determinant $\{ \pm 1\}$. For the inductive step we must distinguish between two cases according as $\operatorname{det} m_{n-1}=\{ \pm 1\}$ or $\{ \pm i\}$.

Case 1. $\operatorname{det} m_{n-1}=\{ \pm 1\}$; then $\xi_{n} \in \mathcal{J}, \xi_{n}^{\prime} \in \overline{\mathcal{J}^{*}}$ by the inductive assumption. There are three subcases.

(a) $T_{n}=V_{j}$; then $\operatorname{det} m_{n}=\{ \pm 1\}$. Of course $\xi_{n+1} \in \mathcal{J}$ by (2.3), but also

$$
\xi_{n+1}^{\prime}=v_{j}^{-1}\left(\xi_{n}^{\prime}\right) \in v_{j}^{-1}\left(\overline{\mathfrak{J}^{*}}\right)=x \circ v_{j} \circ x\left(\overline{\mathfrak{J}^{*}}\right)=\overline{\vartheta_{j}^{*}} \subset \overline{\mathfrak{J}^{*}}
$$

by (2.2) and Lemma 1.1 (v) (vii), hence $\xi_{n+1}$ is reduced. 
(b) $T_{n}=E_{j}$; then $\operatorname{det} m_{n}=\{ \pm i\}$. Of course $\xi_{n+1} \in \mathcal{J}^{*}$ by (2.4), but also

$$
\xi_{n+1}^{\prime}=e_{j}^{-1}\left(\xi_{n}^{\prime}\right) \epsilon e_{j}^{-1}\left(\overline{\mathfrak{J}^{*}}\right)=\varkappa \circ e_{j} \circ x\left(\overline{\mathfrak{J}^{*}}\right)=\overline{\mathcal{E}_{j}} \subset \overline{\mathfrak{J}}
$$

by (2.2) and Lemma $1.1(\mathrm{v})$ (vi), hence $\xi_{n+1}$ is dually reduced.

(c) $T_{n}=C$; then $\operatorname{det} m_{n}=\{ \pm i\}$. Of course $\xi_{n+1} \in \mathcal{J}^{*}$ by (2.4), but also

$$
\xi_{n+1}^{\prime}=c^{-1}\left(\xi_{n}^{\prime}\right) \in c^{-1}\left(\overline{\mathfrak{J}^{*}}\right)=\varkappa \circ \operatorname{cox}\left(\overline{\mathfrak{J}^{*}}\right)=\overline{\mathcal{C}} \subset \overline{\mathfrak{J}}
$$

by (2.2) and Lemma $1.1(\mathrm{v})$ (vi), hence $\xi_{n+1}$ is dually reduced.

Case 2. det $m_{n-1}=\{ \pm i\}$; then $\xi_{n} \in \mathcal{J}^{*}, \xi_{n}^{\prime} \in \overline{\mathfrak{J}}$ by the inductive assumption. The two subcases (a) $T_{n}=V_{j}$, (b) $T_{n}=C$ are treated as in case 1 .

This completes the second step of the proof.

To finish the proof of Theorem 3.4 suppose that the regular chain (3.1) of $\xi$ is not purely periodic, hence $T_{h} \neq T_{h+k}(h \geqslant 0)$. Then we have to obtain a contradiction. We have to distinguish between two cases according as $\operatorname{det} m_{h}=\{ \pm 1\}$ or $\{ \pm i\}$.

Case 1. $\operatorname{det} m_{h}=\{ \pm 1\}$; then $\xi_{h+1} \in \mathcal{J}, \xi_{h+1}^{\prime} \in \overline{J^{*}}$ by the second step of the proof, and $T_{h}=V_{j}$ or $C$ by the very definition of a regular chain.

(a) $T_{h}=V_{j}$; then $\operatorname{det} m_{n-1}=\{ \pm 1\}$. By the second step of the proof $\xi_{h}$ is reduced, in particular $\xi_{h}^{\prime}=v_{j}\left(\xi_{h+1}^{\prime}\right) \in \overline{\mathfrak{J}^{*}}$, and hence $\xi_{h+1}^{\prime} \in v_{j}^{-1}\left(\overline{\mathfrak{J}^{*}}\right)=\varkappa \circ v_{j} \circ \chi\left(\overline{\mathfrak{J}^{*}}\right)=\mathfrak{V}_{j}^{*}$.

(b) $T_{n}=C$; then det $m_{n-1}=\{ \pm i\}$. By the second step of the proof $\xi_{h}$ is dually reduced, in particular $\xi_{h}^{\prime}=c\left(\xi_{n+1}^{\prime}\right) \in \overline{\mathrm{J}}$, and hence $\xi_{h+1}^{\prime} \in c^{-1}(\overline{\mathrm{J}})=\varkappa \circ \circ \circ \xi(\overline{\mathrm{J}})=\overline{\mathrm{C}^{*}}$.

Using the first step of the proof, we obtain from (a), (b) that $T_{h}$ is uniquely determined by the position of $\xi_{h+1}^{\prime}$. However, applying the same argument to $\xi_{h+2 k}$ (also reduced or dually reduced), we conclude that $T_{h}=T_{h+2 t}=T_{h+k}$, since $\operatorname{det} m_{h+2 k}=\operatorname{det} m_{h}=\{ \pm 1\}$ and $\xi_{h+1}^{\prime}=\xi_{h+2 k+1}^{\prime}$ by (3.1). This is the required contradiction.

Case 2. $\operatorname{det} m_{h}=\{ \pm i\}$; then $\xi_{h+1} \in \mathcal{Y}^{*}, \xi_{h+1}^{\prime} \in \overline{\mathfrak{J}}$ by the second step of the proof. The three subcases (a) $T_{h}=V_{j}$, (b) $T_{h}=E_{j}$, (c) $T_{h}=C$ lead to a contradiction as in case 1 .

The dual part of Theorem 3.4 is proved similarly.

\subsection{Inverse periods}

THEOREM 3.5. If $\xi \in \mathbf{C} \backslash \mathbf{Q}(i)$ has the purely periodic regular (dually regular) chain

$$
\overline{T_{0} T_{1} \ldots T_{k-1}} \text {, }
$$

then $\overline{\xi^{\prime}}$ has the purely periodic dually regular (regular) chain

$$
\overrightarrow{T_{k-1} T_{k-2} \ldots T_{0}} .
$$


Proof. To be specific suppose that $\xi$ has the purely periodic regular chain

$$
\overline{T_{0} T_{1} \ldots T_{k-1}} \text {. }
$$

Then $\xi \in \mathcal{J}, \xi^{\prime} \in \overline{\mathcal{J}^{*}}$ are the roots of the quadratic equation (with coefficients in $\mathbf{Z}[i]$, when reduced)

$$
z=t_{0} \circ t_{1} \circ \ldots \circ t_{k-1}(z)
$$

Evidently

$$
\widehat{T_{k-1} T_{k-2} \ldots T_{0}}
$$

is then a purely periodic dually regular chain, which represents a number $\eta \in \mathbf{C} \backslash \mathbf{Q}(i)$, quadratic over $\mathbf{Q}(i)$ and dually reduced. Hence $\eta \in \mathcal{J}^{*}, \eta^{\prime} \in \overline{\mathcal{J}}$ are the roots of the quadratic equation

$$
\begin{aligned}
z=t_{k-1} \circ t_{k-2} \circ \ldots \circ t_{0}(z) & =\left(x \circ t_{k-1}^{-1} \circ x\right) \circ\left(x \circ t_{k-2}^{-1} \circ x\right) \circ \ldots \circ\left(x \circ t_{0}^{-1} \circ x\right)(z) \\
& =\varkappa \circ\left(t_{k-1}{ }^{-1} \circ t_{k-2}{ }^{-1} \circ \ldots \circ t_{0}^{-1}\right) \circ x(z),
\end{aligned}
$$

whence applying $t_{0} \circ t_{1} \circ \ldots \circ t_{k-1} \circ \%$ on both sides,

$$
\bar{z}=t_{0} \circ t_{1} \circ \ldots \circ t_{k-1}(\bar{z}) .
$$

It follows that $\bar{\eta} \in \overline{\mathcal{J}^{*}}, \bar{\eta}^{\prime} \in \mathcal{J}$ are also roots of the equation

$$
z=t_{0} \circ t_{1} \circ \ldots \circ t_{k-1}(z) \text {. }
$$

However, since $\mathfrak{I} \cap \overline{\mathfrak{J}^{*}}=\{0,1\}$, we must have $\overline{\eta^{\prime}}=\xi$, hence $\eta=\overline{\xi^{\prime}}$, which we had to prove.

The dual part of Theorem 3.5 is proved similarly.

\subsection{Special quadratic surds. The Pellian equation}

Suppose $D \in \mathbf{Z}[i]$, where $D$ is not a Gaussian square, and let

$$
\operatorname{ch} \sqrt{D}=T_{0} T_{1} \ldots T_{n} \ldots
$$

be any regular chain of $\sqrt{D}(\arg \sqrt{D} \in[0, \pi[)$. With the notation in section 3.1

$$
\sqrt{D}=\xi_{0}=m_{n}\left(\xi_{n+1}\right)=\frac{p_{1}^{(n)}\left(\sqrt{D}+P_{n+1}\right)+p_{2}^{(n)} Q_{n+1}}{q_{1}^{(n)}\left(\sqrt{D}+P_{n+1}\right)+q_{2}^{(n)} Q_{n+1}}
$$

whence, using that $(1, \sqrt{D})$ is an independent set over $\mathbf{Q}(i)$,

$$
\left(\begin{array}{ll}
p_{1}^{(n)} & D q_{1}^{(n)} \\
q_{1}^{(n)} & p_{1}^{(n)}
\end{array}\right)=M_{n}\left(\begin{array}{cc}
1 & P_{n+1} \\
0 & Q_{n+1}
\end{array}\right)
$$




$$
\begin{aligned}
\left(\begin{array}{ll}
p_{2}^{(n)} & D q_{2}^{(n)} \\
q_{2}^{(n)} & p_{2}^{(n)}
\end{array}\right) & =M_{n}\left(\begin{array}{cc}
0 & \left(D-P_{n+1}^{2}\right) / Q_{n+1} \\
1 & -P_{n+1}
\end{array}\right), \\
\left(\begin{array}{cc}
p_{3}^{(n)} & D q_{3}^{(n)} \\
q_{3}^{(n)} & p_{3}^{(n)}
\end{array}\right) & =M_{n}\left(\begin{array}{cc}
1 & P_{n+1}+\left(D-P_{n+1}^{2}\right) / Q_{n+1} \\
1 & Q_{n+1}-P_{n+1}
\end{array}\right) .
\end{aligned}
$$

Finally, taking determinants, we obtain

$$
\begin{aligned}
& p_{1}^{(n)_{2}}-D q_{1}^{(n) 2}=\varepsilon_{n} Q_{n+1}, \\
& p_{2}^{(n)_{2}}-D q_{2}^{(n) 2}=-\varepsilon_{n}\left(D-P_{n+1}^{2}\right) / Q_{n+1} \\
& p_{3}^{(n) 2}-D q_{3}^{(n) 2}=-\varepsilon_{n}\left(D-\left(P_{n+1}-Q_{n+1}\right)^{2}\right) / Q_{n+1}
\end{aligned}
$$

where

$$
\varepsilon_{n}=\operatorname{det} M_{n}=p_{1}^{(n)} q_{2}^{(n)}-p_{2}^{(n)} \dot{q}_{1}^{(n)} \in\{ \pm 1, \pm i\}
$$

It is an easy consequence of Theorem 2.5 that for any solution $(X, Y) \in Z[i] \times(Z[i] \backslash\{0\})$ of the Diophantine equation

$$
X^{2}-D Y^{2}=\varepsilon, \quad \varepsilon \in \mathcal{U}=\{ \pm 1, \pm i\}
$$

either $X / Y$ or $-X / Y$ is a convergent $p_{j}^{(n)} / q_{j}^{(n)}$ of $\sqrt{D}$. Hence by (3.11), (3.12), (3.13) the complete solution $(X, Y) \in \mathbf{Z}[i] \times(\mathbf{Z}[i] \backslash\{0\})$ of $(3.14)$ consists of

$$
\left\{\varepsilon^{\prime}\left( \pm p_{i}^{(n)}, q_{j}^{(n)}\right) \mid \varepsilon^{\prime} \in \mathcal{U},(j, n) \in S\right\}
$$

where

$$
\mathcal{S}=\left\{(1, n) \mid Q_{n} \in \mathcal{U}\right\} \cup\left\{(2, n) \mid\left(D-P_{n+1}^{2}\right) / Q_{n+1} \in \mathcal{U}\right\} \cup\left\{(3, n) \mid\left(D-\left(P_{n+1}-Q_{n+1}\right)^{2}\right) / Q_{n+1} \in \mathcal{U}\right\} .
$$

To solve equation (3.14) it is not restriction to assume that $D=a+i b, a, b \in \mathbf{N}_{0}$ (otherwise replace $D$ by $-D, \bar{D}$ or $-\bar{D}$ ), and hence that $\sqrt{D}=\alpha+i \beta$ with $\alpha \geqslant \beta \geqslant 0$. It is then an easy consequence of Theorem 3.4, that $\sqrt{D}$ (except for $D=i, 1+i, 1+2 i, 2+i, 3 i, 4 i$ ) has a unique regular chain of the form

$$
\operatorname{ch} \sqrt{D}=V_{1}^{b_{0}} E_{2} \widehat{T_{2} T_{3} \ldots T_{k} T_{k+1}},
$$

where $k$ denotes the shortest period with

$$
\operatorname{det} t_{2} \circ t_{3} \circ \ldots \circ t_{k} \circ t_{k+1}=\{ \pm 1\} \text {. }
$$

It follows easily that a fundamental solution of the Pellian equation

$$
X^{2}-D Y^{2}= \pm 1
$$

is given by

(a) $\left(p_{j+1}^{(l+1)}, q_{j+1}^{(l+1)}\right), j=1,2$, in case $k=3 l$ and $T_{n+l}=S^{j} T_{n} S^{-j}$ for $2 \leqslant n \leqslant 2 l+1$ 3-752903 Acta mathematica 134. Imprimé le 30 Juillet 1975 
and otherwise by

(b) $\left(p_{1}^{(k+1)}, q_{1}^{(k+1)}\right)$.

Also the calculation of (3.16) gives an effective method to decide whether the nonPellian equation

$$
X^{2}-D Y^{2}= \pm i
$$

has solutions, and to determine a fundamental solution if this happens to be the case.

This method of solving equation (3.14) is illustrated by the following examples.

Example 3.1. $D=7+2 i ; \operatorname{ch} \sqrt{7+2 i}$ is of type (a) with $b_{0}=0, k=3 \cdot 13, j=1$ and

$$
T_{2} \ldots T_{14}=V_{1} C V_{1} E_{3} C V_{3} V_{1} V_{2} E_{3} V_{1} V_{2} V_{2} V_{2} \text {. }
$$

Hence a fundamental solution of the Pellian equation is

$$
\left(p_{2}^{(14)}, q_{2}^{(14)}\right)=\left(p_{2}^{(11)}, q_{2}^{(11)}\right)=(17-63 i, 3-24 i)
$$

while the non-Pellian equation has no solutions.

Example 3.2. $D=7+3 i$; ch $\sqrt{7+3} i$ is of type (a) with $b_{0}=0, k=3 \cdot 16, j=2$ and

$$
T_{2} \ldots T_{17}=V_{1} C V_{1} V_{3} V_{1} V_{1} E_{2} V_{1} C V_{3} E_{1} C E_{1} V_{3} V_{3} V_{3}
$$

Hence a fundamental solution of the Pellian equation is

$$
\left(p_{3}^{(17)}, q_{8}^{(17)}\right)=\left(p_{3}^{(13)}, q_{3}^{(13)}\right)=(99-98 i, 28-42 i),
$$

while the non-Pellian equation has no solutions.

Example 3.3. $D=7+4 i$; since

$$
\operatorname{ch} \sqrt{7+4 i}=V_{1}^{0} E_{2} \overrightarrow{V_{1} C E_{2} C E_{2} V_{1} V_{1} \vec{V}_{1}}
$$

is of type (b) with $b_{0}=0, k=8$, a fundamental solution of the Pellian equation is

$$
\left(p_{1}^{(9)}, q_{1}^{(9)}\right)=\left(p_{1}^{(5)}, q_{1}^{(5)}\right)=(-7+4 i,-2+2 \mathrm{i}),
$$

while the non-Pellian equation has no solutions.

Example 3.4. $D=8+7 i$; since

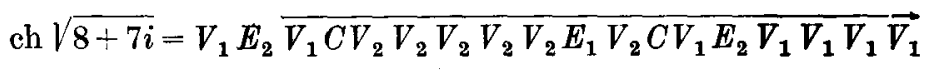

is a type of (b) with $b_{0}=1, k=16$, a fundamental solution of the Pellian equation is

$$
\left(p_{1}^{(17)}, q_{1}^{(17)}\right)=\left(p_{1}^{(11)}, q_{1}^{(11)}\right)=(-13+16 i,-2+6 i),
$$

while a fundamental solution of the non-Pellian equation is

$$
\left(p_{2}^{(8)}, q_{2}^{(8)}\right)=\left(p_{2}^{(3)}, q_{2}^{(3)}\right)=(-3-i,-1) .
$$




\section{Chapter 4}

\section{C-regular and C-dually regular continued fractions}

\subsection{Introduction}

We consider a complex number

$$
\xi_{0}=\frac{1}{2}\left(1+i \alpha_{0}\right), \quad \alpha_{0} \in \mathbf{R} \backslash \mathbf{Q},
$$

lying on the line $\operatorname{Re} z=\frac{1}{2}$, which is an axis of symmetry of Fig. 1 and Fig. $1^{*}$. It follows by Theorem 2.2 that $\xi_{0}$ has precisely one regular chain

$$
\operatorname{ch} \xi_{0}=T_{0} T_{1} T_{2} \ldots
$$

and that

$$
T_{n} \in\left\{V_{1}, E_{1}, C\right\}, \quad n \in \mathbf{N}_{0} .
$$

Hence, collecting powers of $V_{1}$, we have

$$
\operatorname{ch} \xi_{0}=V_{1}^{a_{0}-1} U_{1} V_{1}^{a_{1}-1} C V_{1}^{a_{2}-1} U_{3} V_{1}^{a_{3}-1} C V_{1}^{a_{4}-1} \ldots,
$$

where

$$
a_{0} \in \mathbf{Z}, a_{n} \in \mathbf{N}, U_{2 n-1} \in\left\{E_{1}, C\right\} \text { for } n \in \mathbf{N} \text {. }
$$

Put

$$
\xi_{n}=\left[T_{n} T_{n+1} \ldots\right]=\frac{1}{2}\left(1+i \alpha_{n}\right),
$$

then the relation $\xi_{n}=t_{n}\left(\xi_{n+1}\right)$ is equivalent to

$$
\alpha_{n}= \begin{cases}2+\alpha_{n+1} & \text { if } T_{n}=V_{1} . \\ 1-\frac{2}{1+\alpha_{n+1}} & \text { if } T_{n}=E_{1} . \\ 1+\frac{2}{1+\alpha_{n+1}} & \text { if } T_{n}=C .\end{cases}
$$

Hence corresponding to formula (4.1) we have

$$
\alpha_{0}=2 a_{0}-1+\frac{2 \varepsilon_{1} \mid}{\mid 2 a_{1}}+\frac{2 \mid}{\mid 2 a_{2}}+\frac{2 \varepsilon_{3} \mid}{\mid 2 a_{3}}+\frac{2 \mid}{\mid 2 a_{4}}+\ldots
$$

with

$$
\varepsilon_{2 n-1}=\left\{\begin{array}{ll}
+1 & \text { if } U_{2 n-1}=C, \\
-1 & \text { if } U_{2 n-1}=E_{1},
\end{array} \quad n \in \mathbf{N}\right.
$$

Similarly, by Theorem $2.2^{*}, \xi_{0}$ has precisely one dually regular chain

$$
\operatorname{ch}^{*} \xi_{0}=V_{1}^{b_{9}-1} C V_{1}^{b_{1}-1} U_{2} V_{1}^{b_{9}-1} C V_{1}^{b_{3}-1} U_{4} V_{1}^{b_{4}-1} \ldots,
$$

where $b_{0} \in \mathbf{Z}, b_{n} \in \mathbf{N}, U_{2 n} \in\left\{E_{1}, C\right\}$ for $n \in \mathbf{N}$, and correspondingly

$$
\alpha_{0}=2 b_{0}-1+\frac{2 \mid}{\mid 2 b_{1}}+\frac{2 \varepsilon_{2} \mid}{\mid 2 b_{2}}+\frac{2 \mid}{\mid 2 b_{3}}+\frac{2 \varepsilon_{4} \mid}{\mid 2 b_{4}}+\ldots,
$$


with

$$
\varepsilon_{2 n}=\left\{\begin{array}{ll}
+1 & \text { if } U_{2 n}=C, \\
-1 & \text { if } U_{2 n}=E_{1},
\end{array} \quad n \in \mathbf{N}\right.
$$

A continued fraction (4.2) with $a_{0} \in \mathbf{Z}, a_{n} \in \mathbf{N}, \varepsilon_{2 n-1} \in\{-1,+1\}$ for $n \in \mathbf{N}$ is called $C$ regular. Similarly, a continued fraction $\left(4.2^{*}\right)$ with $b_{0} \in \mathbf{Z}, b_{n} \in \mathbf{N}, \varepsilon_{2 n} \in\{-1,+1\}$ for $n \in \mathbf{N}$ is called $C$-dually regular.

By the correspondences $(4.1),(4.2)$ and $\left(4.1^{*}\right),\left(4.2^{*}\right)$ the following results from Theorems $2.1,2.1^{*}, 2.2,2.2^{*}, 3.1,3.1^{*}, 3.2,3.2^{*}$.

TH Еов м 4.1. Any C-regular (C-dually regular) continued fraction converges to some $\alpha \in \mathbf{R} \backslash \mathbf{Q}$.

THEOR EM 4.2. Any $\alpha \in \mathbf{R} \backslash \mathbf{Q}$ has precisely one C-regular (C-dually regular) continued fraction expansion.

THEOR м 4.3. A periodic C-regular (C-dually regular) continued fraction converges to an $\alpha \in \mathbf{R} \backslash \mathbf{Q}$ which is quadratic over $\mathbf{Q}$.

THEOREM 4.4. Every $\alpha \in \mathbf{R} \backslash \mathbf{Q}$ which is quadratic over $\mathbf{Q}$ has a periodic $C$-regular (C. dually regular) continued fraction expansion.

An $\alpha \in \mathbf{R} \backslash \mathbf{Q}$, quadratic over $\mathbf{Q}$ and with conjugate $\alpha^{\prime}$, is called $C$-reduced if $\alpha>0$, $\alpha^{\prime}<-1$, and is called $C$-dually reduced if $\alpha>1, \alpha^{\prime}<0$.

A $C$-regular ( $C$-dually regular) continued fraction $(4.2)\left(\left(4.2^{*}\right)\right)$ is called purely periodic if the sequences $a_{0}, a_{1}, a_{2}, \ldots$ and $\varepsilon_{1}, \varepsilon_{3}, \varepsilon_{5}, \ldots\left(b_{0}, b_{1}, b_{2}, \ldots\right.$ and $\left.\varepsilon_{2}, \varepsilon_{4}, \varepsilon_{6}, \ldots\right)$ are both purely periodic.

Theorems 3.3, 3.4, 3.5 then specialize as follows.

THEOREM 4.5. An $\alpha \in \mathbf{R} \backslash \mathbf{Q}$, quadratic over $\mathbf{Q}$, is C-reduced (C-dually reduced) if and only if the $C$-regular ( $C$-dually regular) continued fraction of $\alpha$ is purely periodic.

THEOR $\mathbf{M}$ 4.6. If $\alpha \in \mathbf{R} \backslash \mathbf{Q}$ has the purely periodic $C$-regular continued fraction (4.2) with

$$
\begin{aligned}
& a_{0}, a_{1}, a_{2}, \ldots=\widetilde{a_{0}, a_{1}, \ldots, a_{2 k-1}}, \\
& \varepsilon_{1}, \varepsilon_{3}, \varepsilon_{5}, \ldots=\overline{\varepsilon_{1}, \varepsilon_{3}, \ldots, \varepsilon_{2 k-1}},
\end{aligned}
$$


then $-\alpha^{\prime}$ (where $\alpha^{\prime}$ is the conjugate of $\alpha$ in $\mathbf{Q}(\alpha)$ over $\mathbf{Q}$ ) has the purely periodic $\mathbf{C}$ dually regular continued fraction $\left(4.2^{*}\right)$ with

$$
\begin{aligned}
b_{0}, b_{1}, b_{2}, \ldots & =\overrightarrow{a_{2 k-1}, a_{2 k-3}, \ldots, \overrightarrow{a_{0}}}, \\
\varepsilon_{2}, \varepsilon_{4}, \varepsilon_{6}, \ldots & =-\frac{\varepsilon_{2 k-1}, \varepsilon_{2 k-3}, \ldots, \varepsilon_{1}}{},
\end{aligned}
$$

and conversely.

\subsection{C-equivalence and C-duality}

A (real) unimodular map $\varphi: t \mapsto(a t+b)(c t+d)^{-1}$ with $a, b, c, d \in \mathbf{Z}, a d-b c= \pm \mathbf{l}$, is called $C$-unimodular if either $a \equiv d \equiv 0(\bmod 2), b \equiv c \equiv 1(\bmod 2)$ or $a \equiv d \equiv 1(\bmod 2), b \equiv c \equiv 0(\bmod$ 2). It is well known that the set $\Gamma_{C}$ of all $C$-unimodular maps is a group, which is generated by the maps $\varphi_{0}: t \mapsto t+2, \varphi_{1}: t \mapsto t^{-1}$.

Correspondingly, $\alpha, \beta \in \mathbf{R} \cup\{\infty\}$ are called $C$-equivalent $(\alpha \approx \beta)$ if there exists a map $\varphi \in \Gamma_{C}$ with $\beta=\varphi(\alpha)$.

Notice that $\mathbf{Q}$ consists of two $C$-equivalence classes $\mathbf{Q}_{0}, \mathbf{Q}_{\mathbf{1}}$, where (for $j=0,1$ )

$$
\mathbf{Q}_{\jmath}=\{p / q \mid(p, q) \in \mathbf{Z} \times \mathbf{N}, \operatorname{gcd}(p, q)=1, p+q \equiv j(\bmod 2)\} .
$$

For any $\alpha \in \mathbf{R} \cup\{\infty\}$, the $C$-dual $\alpha^{*}$ of $\alpha$ is defined as $\alpha^{*}=(\alpha+1) /(\alpha-1)$.

Notice that $\left(\alpha^{*}\right)^{*}=\alpha$ and that $\alpha \approx \beta \Leftrightarrow \alpha^{*} \approx \beta^{*}$ for all $\alpha, \beta \in \mathbf{R} \cup\{\infty\}$. Notice also that $\alpha \approx \beta$ if and only if $\frac{1}{2}(1+i \alpha), \frac{1}{2}(1+i \beta)$ are properly equivalent, and that $\alpha^{*} \approx \beta$ if and only if $\frac{1}{2}(1+i \alpha), \frac{1}{2}(1+i \beta)$ are improperly equivalent.

Now Theorem 2.3 specializes as follows.

THEOR EM 4.7. $\alpha, \beta \in \mathbf{R} \backslash \mathbf{Q}$ are $C$-equivalent if and only if the C-regular continued fractions of $\alpha, \beta$ are of the form

$$
\begin{aligned}
& \alpha=2 a_{0}-1+\frac{2 \varepsilon_{1} \mid}{\mid 2 a_{1}}+\frac{2 \mid}{\mid 2 a_{2}}+\frac{2 \varepsilon_{3} \mid}{\mid 2 a_{3}}+\frac{\mid 2}{\mid 2 a_{4}}+\ldots, \\
& \beta=2 c_{0}-1+\frac{2 \delta_{1} \mid}{\mid 2 c_{1}}+\frac{2 \mid}{\mid 2 c_{2}}+\frac{2 \delta_{3} \mid}{\mid 2 c_{3}}+\frac{2 \mid}{\mid 2 c_{4}}+\ldots,
\end{aligned}
$$

with (for suitable $h, k \in \mathbf{N}$ )

$$
a_{2 h-1+n}=c_{2 k-1+n}, \varepsilon_{2 h-1+2 n}=\delta_{2 k-1+2 n} \quad \text { for all } n \in \mathbf{N} \text {. }
$$

Of course, Theorems $2.3^{*}, 2.4$ have similar specializations. 


\subsection{C-convergents and $C$-dual convergents}

For any $\alpha \in \mathbf{R} \backslash \mathbf{Q}$ we write its $C$-regular continued fraction (4.2) reduced form as

$$
\alpha=2 a_{0}-1+\frac{\varepsilon_{1} \mid}{\mid a_{1}}+\frac{1 \mid}{\mid 2 a_{2}}+\frac{\varepsilon_{3} \mid}{\mid a_{3}}+\frac{1 \mid}{\mid 2 a_{4}}+\ldots
$$

and we call the convergents $p_{n} / q_{n}, n \in \mathbf{N}_{0}$, of (4.3) the $C$-convergents of $\alpha$.

Similarly we write its $C$-dually regular continued fraction $\left(4.2^{*}\right)$ in reduced form as

$$
\alpha=2 b_{0}-1+\frac{1 \mid}{\mid b_{1}}+\frac{\varepsilon_{2} \mid}{\mid 2 b_{2}}+\frac{1 \mid}{\mid b_{3}}+\frac{\varepsilon_{4} \mid}{\mid 2 b_{4}}+\ldots
$$

and we call the convergents $p_{n}^{*} / q_{n}^{*}, n \in \mathbf{N}_{0}$, of $\left(4.3^{*}\right)$ the $C$-dual convergents of $\alpha$.

The following result is an immediate consequence of the recursion formulas of continued fractions.

Theorem 4.8. For any $\alpha \in \mathbf{R} \backslash \mathbf{Q}$ with $C$-convergents $p_{n} / q_{n}$ and $C$-dual convergents $p_{n}^{*} / q_{n}^{*}$, the following is valid:

(i) $p_{2 n} / q_{2 n}, p_{2 n}^{*} / q_{2 n}^{*} \in \mathbf{Q}_{0}, p_{2 n+1} / q_{2 n+1}, p_{2 n+1}^{*} / q_{2 n+1}^{*} \in \mathbf{Q}_{1}$ for $n \in \mathbf{N}_{0}$,

(ii) $p_{n} q_{n+1}-p_{n+1} q_{n}= \pm 1, p_{n}^{*} q_{n+1}^{*}-p_{n+1}^{*} q_{n}^{*}= \pm 1 \quad$ for $n \in \mathbf{N}_{0}$,

(iii) $1=q_{0}<2 q_{1}<q_{2}<2 q_{3}<q_{4}<2 q_{5}<\ldots$,

(iii*) $1=q_{0}^{*}<q_{1}^{*}<q_{2}^{*}<q_{3}^{*}<\ldots$,

(iv) $\left|q_{0} \alpha-p_{0}\right|>\left|q_{1} \alpha-p_{1}\right|>\left|q_{2} \alpha-p_{2}\right|>\ldots$,

(iv $\left.{ }^{*}\right)\left|q_{0}^{*} \alpha-p_{0}^{*}\right|>2\left|q_{1}^{*} \alpha-p_{1}^{*}\right|>\left|q_{2}^{*} \alpha-p_{2}^{*}\right|>2\left|q_{3}^{*} \alpha-p_{3}^{*}\right|>\ldots$

The following theorems generalize classical theorems of Legendre on regular continued fractions, and can be proved similarly.

THEOREM 4.9. The sequence $p_{n} / q_{n}, n \in \mathbf{N}_{0}$, of C-convergents of $\alpha \in \mathbf{R} \backslash \mathbf{Q}$ is characterized as the maximal sequence $p_{n} / q_{n}$ satisfying conditions (i), (iii), (iv) of Theorem 4.8 .

The sequence $p_{n}^{*} / q_{n}^{*}, n \in \mathbf{N}_{0}$, of C-dual convergents of $\alpha \in \mathbf{R} \backslash \mathbf{Q}$ is characterized as the maximal sequence $p_{n}^{*} / q_{n}^{*}$ satisfying conditions (i), (iii*), (iv*) of Theorem 4.8 .

TheOREM 4.10. Let $\alpha \in \mathbf{R} \backslash \mathbf{Q}$. Any $p / q \in \mathbf{Q}_{j}(j=0,1)$ satisfying the inequality

$$
|\alpha-p / q|<\left(c_{j} q^{2}\right)^{-1}, \quad\left(c_{0}=\frac{3}{2}, c_{1}=2\right),
$$

is a C-convergent of $\alpha$. 
Any $p / q \in \mathbf{Q}_{j}(j=0,1)$ satisfying the inequality

$$
|\alpha-p / q|<\left(c_{j}^{*} q^{2}\right)^{-1}, \quad\left(c_{0}^{*}=2, c_{1}^{*}=\frac{3}{2}\right)
$$

is a $C$-dual convergent of $\alpha$.

\subsection{The C-approximation constant}

For any $\alpha \in \mathbf{R} \backslash \mathbf{Q}$ we define

$$
\begin{aligned}
& d_{j}(\alpha)=\limsup _{p / q \in \mathbf{Q}_{j}}(q|q \alpha-p|)^{-1},(j=0,1), \\
& d(\alpha)=\max \left(d_{0}(\alpha), \frac{1}{2} d_{1}(\alpha)\right),
\end{aligned}
$$

and we call $d(\alpha)$ the $C$-approximation constant of $\alpha$.

It was proved by W. T. Scott [34], that $d_{0}(\alpha) \geqslant 1$ for all $\alpha \in \mathbf{R} \backslash \mathbf{Q}$, and by R. M. Robinson [31], that $d_{1}(\alpha) \geqslant 2$ for all $\alpha \in \mathbf{R} \backslash \mathbf{Q}$. See also L. C. Eggan [11].

The following result is an easy consequence of Theorems 4.8, 4.9.

TH EOREM 4.11. For any $\alpha \in \mathbf{R} \backslash \mathbf{Q}$ with $C$-regular continued fraction (4.2) and $C$-dually regular continued fraction $\left(4.2^{*}\right)$, we have

$$
\begin{aligned}
& d_{0}(\alpha)=\lim _{n} \sup d_{2 n}=\underset{n}{\lim \sup } d_{2 n}^{*}, \\
& d_{1}(\alpha)=\limsup _{n} d_{2 n-1}=\underset{n}{\lim \sup } d_{2 n-1}^{*},
\end{aligned}
$$

where

$$
d_{n}=\left(q_{n}\left|q_{n} \alpha-p_{n}\right|\right)^{-1}, d_{n}^{*}=\left(q_{n}^{*}\left|q_{n}^{*} \alpha-p_{n}^{*}\right|\right)^{-1}
$$

are given by

$$
\begin{aligned}
2 d_{2 n}=2 a_{2 n+1}+\frac{2 \mid}{\mid 2 a_{2 n+2}}+\frac{2 \varepsilon_{2 n+3} \mid}{\mid 2 a_{2 n+3}}+\frac{2 \mid}{\mid 2 a_{2 n+4}}+\ldots & \\
& +\frac{2 \varepsilon_{2 n+1} \mid}{\mid 2 a_{2 n}}+\frac{2 \mid}{\mid 2 a_{2 n-1}}+\frac{2 \varepsilon_{2 n-1} \mid}{\mid 2 a_{2 n-2}}+\ldots+\frac{2 \varepsilon_{3} \mid}{\mid 2 a_{2}}+\frac{2 \mid}{\mid 2 a_{1}}, \\
d_{2 n-1}=2 a_{2 n}+\frac{2 \varepsilon_{2 n+1} \mid}{\mid 2 a_{2 n+1}}+\frac{2 \mid}{\mid 2 a_{2 n+2}}+ & \frac{2 \varepsilon_{2 n+3} \mid}{\mid 2 a_{2 n+3}}+\ldots \\
& +\frac{2 \mid}{\mid 2 a_{2 n-1}}+\frac{2 \varepsilon_{2 n-1} \mid}{\mid 2 a_{2 n-2}}+\frac{2 \mid}{\mid 2 a_{2 n-3}}+\ldots+\frac{2 \varepsilon_{3} \mid}{\mid 2 a_{2}}+\frac{2 \mid}{\mid 2 a_{1}},
\end{aligned}
$$




$$
\begin{aligned}
& 2 d_{2 n}^{*}=2 b_{2 n+1}+\frac{2 \varepsilon_{2 n+8} \mid}{\mid 2 b_{2 n+2}}+\frac{2 \mid}{\mid 2 b_{2 n+3}}+\frac{2 \varepsilon_{2 n+4} \mid}{\mid 2 b_{2 n+4}}+\ldots \\
& +\frac{2 \mid}{\mid 2 b_{2 n}}+\frac{2 \varepsilon_{2 n} \mid}{\mid 2 b_{2 n-1}}+\frac{2 \mid}{\mid 2 b_{2 n-2}}+\ldots+\frac{2 \mid}{\mid 2 b_{2}}+\frac{2 \varepsilon_{2} \mid}{\mid 2 b_{1}}, \\
& d_{2 n-1}^{*}=2 b_{2 n}+\frac{2 \mid}{\mid 2 b_{2 n+1}}+\frac{2 \varepsilon_{2 n+2}}{\mid 2 b_{2 n+2}}+\frac{2 \mid}{\mid 2 b_{2 n+3}}+\ldots \\
& +\frac{2 \varepsilon_{2 n} \mid}{\mid 2 b_{2 n-1}}+\frac{2 \mid}{\mid 2 b_{2 n-2}}+\frac{2 \varepsilon_{2 n-2} \mid}{\mid 2 b_{2 n-3}}+\ldots+\frac{2 \mid}{\mid 2 b_{2}}+\frac{2 \varepsilon_{2} \mid}{\mid 2 b_{1}} .
\end{aligned}
$$

Corollar y. For any $\alpha, \beta \in \mathbf{R} \backslash \mathbf{Q}$,

$$
\begin{aligned}
& \alpha \approx \beta \Rightarrow d_{0}(\alpha)=d_{0}(\beta), d_{1}(\alpha)=d_{1}(\beta), d(\alpha)=d(\beta), \\
& \alpha^{*} \approx \beta \Rightarrow 2 d_{0}(\alpha)=d_{1}(\beta), d_{1}(\alpha)=2 d_{0}(\beta), d(\alpha)=d(\beta) .
\end{aligned}
$$

Example 4.1. It follows from Theorem 4.11 that for

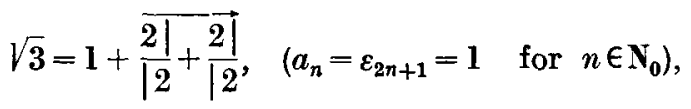

we have

$$
d_{0}(\sqrt{3})=\sqrt{3}, d_{1}(\sqrt{3})=2 \sqrt{3}, d(\sqrt{3})=\sqrt{3}
$$

Example 4.2. It follows from Theorem 4.11 that for

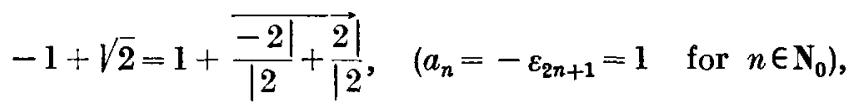

we have

$$
d_{0}(-1+\sqrt{2})=\sqrt{2}, d_{1}(-1+\sqrt{2})=2 \sqrt{2}, d(-1+\sqrt{2})=\sqrt{2}
$$

Example 4.3. It follows from Theorem 4.11 and its corollary that for

$$
e=3+\frac{-1 \mid}{\mid 3}+\frac{1 \mid}{\mid 2}+\frac{-1 \mid}{\mid 5}+\frac{1 \mid}{\mid 2}+\frac{-1 \mid}{\mid 7}+\frac{1 \mid}{\mid 2}+\frac{-1 \mid}{\mid 9}+\ldots
$$

we have

$$
d_{0}(e)=\infty, d_{1}(e)=2, d_{0}((e+1) /(e-1))=1, d_{1}((e+1) /(e-1))=\infty .
$$

In chapter 5 we shall extend the results of Markoff-Hurwitz by finding all $C$-approximation constants $<2$. 


\subsection{Ergodic theory}

Let

$$
\left.X_{1}=\right] 0,2\left[\backslash \mathbf{Q}, X_{2}=\right] 1,3[\backslash \mathbf{Q}
$$

and let $X$ be the disjoint union of $X_{1}$ and $X_{2}$. A normalized measure $\mu$ on $X$ is defined by

$$
\begin{aligned}
& \mu(] 0, x_{1}[)=\frac{1}{2 \log 3} \log \left(1+x_{1}\right), \quad \text { for } x_{1} \in X_{1}, \\
& \mu(] 1, x_{2}[)=\frac{1}{2 \log 3} \log x_{2}, \quad \text { for } x_{2} \in X_{2} .
\end{aligned}
$$

Further let $T: X \rightarrow X$ be given by

$$
\begin{aligned}
& x_{1}=1+\frac{2 \varepsilon_{1}\left(x_{1}\right) \mid}{\mid 2 a_{1}\left(x_{1}\right)}+\frac{2 \mid}{\mid 2 a_{2}\left(x_{1}\right)}+\frac{2 \varepsilon_{3}\left(x_{1}\right) \mid}{\mid 2 a_{3}\left(x_{1}\right)}+\ldots \mapsto T\left(x_{1}\right)=1+\frac{2 \mid}{\mid 2 a_{2}\left(x_{1}\right)}+\frac{2 \varepsilon_{3}\left(x_{1}\right) \mid}{\mid 2 a_{3}\left(x_{1}\right)}+\ldots \in X_{2}, \\
& x_{2}=1+\frac{2 \mid}{\mid 2 b_{1}\left(x_{2}\right)}+\frac{2 \varepsilon_{2}\left(x_{2}\right) \mid}{\mid 2 b_{2}\left(x_{2}\right)}+\frac{2 \mid}{\mid 2 b_{3}\left(x_{2}\right)}+\ldots \mapsto T\left(x_{2}\right)=1+\frac{2 \varepsilon_{2}\left(x_{2}\right) \mid}{\mid 2 b_{2}\left(x_{2}\right)}+\frac{2 \mid}{\mid 2 b_{3}\left(x_{2}\right)}+\ldots \in X_{1} .
\end{aligned}
$$

A simple computation shows that $T$ acts as a measure preserving transformation on $(X, \mu)$, i.e.

$$
\begin{array}{ll}
\mu\left(T^{-1}(] 0, x_{1}[)\right)=\mu(] 0, x_{1}[) & \text { for all } x_{1} \in X_{1}, \\
\mu\left(T^{-1}(] 1, x_{2}[)\right)=\mu(] 1, x_{2}[) & \text { for all } x_{2} \in X_{2} .
\end{array}
$$

Further the argument used by C. Ryll-Nardzewski [32] shows that $T$ is an indecomposable transformation on $(X, \mu)$, i.e. any measurable subset $E \subseteq X$ with $T^{-1}(E)=E$ has $\mu$ measure 0 or 1 .

Hence by the individual ergodic theorem we get the following analogue of a theorem of C. Ryll-Nardzewski [32]; also Corollaries 2, 3 represent analogues of well-known results of P. Lévy and A. Khintchine.

Theormi 4.12. For any $f: X \rightarrow \mathbf{R}, f \in L^{1}(X, \mu)$, we have

$$
\lim _{N \rightarrow \infty} \frac{1}{N} \sum_{n=0}^{N-1} f\left(T^{n}(x)\right)=\frac{1}{2 \log 3}\left\{\int_{0}^{2} \frac{f\left(x_{1}\right)}{1+x_{1}} d x_{1}+\int_{1}^{3} \frac{f\left(x_{2}\right)}{x_{2}} d x_{2}\right\}
$$

for almost all $x \in X$ (with respect to $\mu$ ). 
Coroldary 1. For almost all $x_{2} \in X_{1}, x_{1} \in X_{2}$, we have

$$
\begin{aligned}
& p\left(\varepsilon_{2 n-1}\left(x_{1}\right)=-1\right)=p\left(\varepsilon_{2 n}\left(x_{2}\right)=-1\right)=(\log 2) /(\log 3), \\
& p\left(\varepsilon_{2 n-1}\left(x_{1}\right)=1\right)=p\left(\varepsilon_{2 n}\left(x_{2}\right)=1\right)=(\log 3-\log 2) /(\log 3),
\end{aligned}
$$

where $p\left(c_{n}=c\right)$ denotes the frequency of $c$ in the sequence $\left(c_{n}\right), n \in \mathbf{N}$.

Proof. Apply Theorem 4.12 for $f=\chi_{A_{k}}(k=1,2)$, where $\chi_{A_{k}}$ is the indicator function of $A_{k}$, and

$$
\left.A_{1}=\right] 0,1\left[\subseteq X_{1}, A_{2}=\right] 1,2\left[\subseteq X_{1}\right.
$$

Corolla R 2. For almost all $x_{1} \in X_{1}, x_{2} \in X_{2}$, and all $m \in \mathbf{N}$ we have

$$
\begin{gathered}
p\left(a_{2 n-1}\left(x_{1}\right)=m\right)=p\left(a_{2 n}\left(x_{1}\right)=m\right)=p\left(b_{2 n-1}\left(x_{2}\right)=m\right) \\
=p\left(b_{2 n}\left(x_{2}\right)=m\right)=\frac{1}{\log 3} \log \frac{(2 m+1)^{2}}{(2 m-1)(2 m+3)} .
\end{gathered}
$$

Proof. Apply Theorem 4.12 for $f=\chi_{A_{k}},(k=1,2)$, where

$$
\begin{gathered}
\left.A_{1}=\right] 1-\frac{1}{m}, 1-\frac{1}{m-1}[\cup] 1+\frac{1}{m+1}, 1+\frac{1}{m}\left[\subseteq X_{1},\right. \\
\left.A_{2}=\right] 1+\frac{2}{2 m+1}, 1+\frac{2}{2 m-1}\left[\subseteq X_{2} .\right.
\end{gathered}
$$

Corollary 3. For almost all $x_{1} \in X_{1} x_{2} \in X_{2}$, we have

$$
\begin{aligned}
\lim _{N \rightarrow \infty} & \left(a_{1}\left(x_{1}\right) a_{3}\left(x_{1}\right) \ldots a_{2 N-1}\left(x_{1}\right)\right)^{1 / N} \\
& =\lim _{N \rightarrow \infty}\left(a_{2}\left(x_{1}\right) a_{4}\left(x_{1}\right) \ldots a_{2 N}\left(x_{1}\right)\right)^{1 / N} \\
& =\lim _{N \rightarrow \infty}\left(b_{1}\left(x_{2}\right) b_{3}\left(x_{2}\right) \ldots b_{2 N-1}\left(x_{2}\right)\right)^{1 / N} \\
& =\lim _{N \rightarrow \infty}\left(b_{2}\left(x_{2}\right) b_{4}\left(x_{2}\right) \ldots b_{2 N}\left(x_{2}\right)\right)^{1 / N} \\
& =\prod_{m=1}^{\infty}\left(1+\frac{4}{(2 m-1)(2 m+3)}\right)^{\log m / \log 3} .
\end{aligned}
$$

Proof. Apply Theorem 4.12 for $f=f_{k},(k=1,2)$, where

$$
\begin{aligned}
& f_{1}: x_{1} \mapsto \log a_{1}\left(x_{1}\right), x_{2} \mapsto 0, \\
& f_{2}: x_{1} \mapsto 0, x_{2} \mapsto \log b_{1}\left(x_{2}\right) .
\end{aligned}
$$

Theorem 4.12 and its corollaries can be refined by the method of C. de Vroedt [35]. 


\section{Chapter 5}

\section{C-minimum of binary quadratic forms}

\subsection{Indefinite quadratic forms}

Let

$$
f:(x, y) \mapsto f(x, y)=\alpha x^{2}+\beta x y+\gamma y^{2}, \alpha, \beta, \gamma \in \mathbf{R},
$$

be a quadratic form with $\alpha \neq 0$ and discriminant $\delta=\delta(f)=\beta^{2}-4 \alpha \gamma>0$. The first and second roots $\vartheta_{1}(f)$ and $\vartheta_{2}(f)$ of $f$ are defined as follows,

$$
\vartheta_{1}(f)=(-\beta+\sqrt{\delta}) /(2 \alpha), \vartheta_{2}(f)=(-\beta-\sqrt{\delta}) /(2 \alpha) ;
$$

notice that

$$
\vartheta_{1}(-f)=\vartheta_{2}(f), \vartheta_{2}(-f)=\vartheta_{1}(f)
$$

In the sequel we shall only consider indefinite forms $f$ with both roots $\vartheta_{1}(f), \vartheta_{\mathbf{2}}(f) \in \mathbf{R} \backslash \mathbf{Q}$.

Let $\Gamma_{C}^{\prime}$ denote the group of all matrices

$$
M=\left(\begin{array}{ll}
a & b \\
c & d
\end{array}\right), a, b, c, d \in \mathbf{Z}, a d-b c= \pm 1
$$

with either $a \equiv d \equiv 0(\bmod 2), b \equiv c \equiv 1(\bmod 2)$ or $a \equiv d \equiv 1(\bmod 2), b \equiv c \equiv 0(\bmod 2)$.

Two forms $f, g$ are called $C$-equivalent $(f \approx g)$ if there exists a matrix $M \in \Gamma_{C}^{\prime}$ with $g(x, y)=$ $f(a x+b y, c x+d y)$; notice that (with the notation of section 4.2)

$$
f \approx g \Rightarrow \vartheta_{1}(f) \approx \vartheta_{1}(g), \vartheta_{2}(f) \approx \vartheta_{2}(g) .
$$

For any form $f$ the $C$-dual form $f^{*}$ is defined by $f^{*}(x, y)=\frac{1}{2} f(x+y, x-y)$; notice that

$$
\vartheta_{1}\left(f^{*}\right)=\left(\vartheta_{1}(f)\right)^{*}, \vartheta_{2}\left(f^{*}\right)=\left(\vartheta_{2}(f)\right)^{*}
$$

Using the special notation (for $j=0,1$ )

$$
\mathbf{Z}_{j}^{2}=\left\{(x, y) \in \mathbf{Z}^{2} \backslash\{(0,0)\} \mid \operatorname{ged}(x, y)=1, x+y \equiv j(\bmod 2)\right\},
$$

we define

$$
\begin{gathered}
v_{j}=v_{j}(f)=\inf _{(x, y) \in \mathbf{Z}_{j}^{\mid}}|f(x, y)|,(j=0,1), \\
\nu=v(f)=\min \left(v_{0}(f), 2 v_{1}(f)\right),
\end{gathered}
$$

and we call $v(f)$ the $C$-minimum of $f$. 
Lемм A 5.1. Suppose that $f \approx g, \lambda \in \mathbf{R}$. Then

(i) $\left(f^{*}\right)^{*}=f, f^{*} \approx g^{*}$,

(ii) $(\lambda f)^{*}=\lambda f^{*}, \lambda f \approx \lambda g$,

(iii) $\delta\left(f^{*}\right)=\delta(f), \delta(f)=\delta(g), \delta(\lambda f)=\lambda^{2} \delta(f)$,

(iv) $v_{j}(\lambda f)=|\lambda| \nu_{j}(f), v(\lambda f)=|\lambda| v(f)$,

(v) $\nu_{j}(f)=v_{j}(g), v(f)=v(g)$,

(vi) $v_{0}(f)=2 v_{1}\left(f^{*}\right), 2 v_{1}(f)=v_{0}\left(f^{*}\right), \nu(f)=v\left(f^{*}\right)$.

Proof. (i)-(iv) are obvious; (v) follows from the fact that for $M \in \Gamma_{C}^{\prime},(x, y) \mapsto(a x+b y$, $c x+d y)$ maps $\mathbf{Z}_{j}^{2}$ onto $\mathbf{Z}_{j}^{2},(j=0,1)$. Similarly (vi) follows from the fact that $(x, y) \mapsto(x+y$, $x-y)$ maps $\mathbf{Z}_{0}^{2}$ onto $2 \mathbf{Z}_{1}^{2}$ and $\mathbf{Z}_{1}^{2}$ onto $\mathbf{Z}_{0}^{2}$.

LEMMA 5.2. Suppose that $f(a, c)=\alpha^{\prime}>0,(a, c) \in \mathbf{Z}_{1}^{2}$. Then there exist $b, d \in \mathbf{Z}$ with $\left(\begin{array}{ll}a & b \\ c & d\end{array}\right) \in \Gamma_{C}^{\prime}$, such that

$$
f(a x+b y, c x+d y)=\alpha^{\prime} x^{2}+\beta^{\prime} x y+\gamma^{\prime} y^{2}
$$

and

$$
2 \alpha^{\prime} \leqslant \beta^{\prime} \leqslant 4 \alpha^{\prime}
$$

Proof. Analogous to that of Lemma 1, chapter II of [4].

LEMMA 5.3. Let $\vartheta$ be any of the (irrational) roots of $f$. Then

A. $d(\vartheta) \leqslant \delta^{1 / 2}(v(f))^{-1}$.

B. $d(\vartheta)=\delta^{1 / 2}(v(f))^{-1} \quad$ if $\alpha, \beta, \gamma \in \mathbf{Q}$;

in this case $f$ attains its $C$-minimum, i.e. $\exists(x, y) \in \mathbf{Z}_{0}^{2}$ with $|f(x, y)|=v(f) \vee \exists(x, y) \in \mathbf{Z}_{1}^{2}$ with $|f(x, y)|=\frac{1}{2} v(f)$.

C. If, in addition to $B$, f has the following property,

$\left(^{*}\right)\left[\exists(x, y) \in \mathbf{Z}_{0}^{2}\right.$ with $f(x, y)=v(f) \vee \exists(x, y) \in \mathbf{Z}_{1}^{2}$ with $\left.f(x, y)=\frac{1}{2} v(f)\right]$

$\wedge\left[\exists(x, y) \in \mathbf{Z}_{0}^{2}\right.$ with $f(x, y)=-v(f) \vee \exists(x, y) \in \mathbf{Z}_{1}^{2}$ with $\left.f(x, y)=-\frac{1}{2} v(f)\right]$,

then either

$$
|\vartheta-p / q|<\left(d(\vartheta) q^{2}\right)^{-1} \quad \text { for infinitely many } p / q \in \mathbf{Q}_{0}
$$

or

$$
|\vartheta-p / q|<\left(2 d(\vartheta) q^{2}\right)^{-1} \quad \text { for infinitely many } p / q \in \mathbf{Q}_{\mathbf{1}}
$$


Proof. Analogous to that of Lemma 4, chapter II of [4]; notice that our $d(\vartheta)$, as defined in section 4.4, corresponds to Cassels' $(v(\vartheta))^{-1}$.

ThEOREM 5.1. (Isolation theorem.) Suppose that $f(x, y)=\alpha x^{2}+\beta x y+\gamma y^{2}$, with $\alpha, \beta, \gamma \in \mathbf{Q}$ and irrational roots, satisfies condition $\left({ }^{*}\right)$ of Lemma 5.3 C. Then there exist $\nu^{\prime}<\nu(f)$ and $\varepsilon_{0}>0$, depending only on $\alpha, \beta, \gamma$ such that

$$
\nu(g)<v^{\prime}
$$

for all forms $g$, not proportional to $f$, and of the shape

$$
g(x, y)=\alpha^{\prime} x^{2}+\beta^{\prime} x y+\gamma^{\prime} y^{2}
$$

with

$$
\left|\alpha-\alpha^{\prime}\right|<\varepsilon_{0},\left|\beta-\beta^{\prime}\right|<\varepsilon_{0},\left|\gamma-\gamma^{\prime}\right|<\varepsilon_{0} .
$$

Proof. Analogous to that of Theorem I, chapter II of [4].

\subsection{A diophantine equation}

We consider the following system of equations

$$
x_{1}+x_{2}=2 y_{1} y_{2}, 2 x_{1} x_{2}=y_{1}^{2}+y_{2}^{2}
$$

first studied by L. Ya. Vulakh [36] in the equivalent form

$$
y_{1}^{2}+y_{2}^{2}+2 x^{2}=4 y_{1} y_{2} x
$$

A solution $\left(x_{1}, x_{2} ; y_{1}, y_{2}\right)=\left(\Lambda_{1}, \Lambda_{2} ; M_{1}, M_{2}\right) \in N^{4}$ of $(5.4)$ is called singular if $\Lambda_{1}=\Lambda_{2}$ or $\mathrm{M}_{1}=\mathrm{M}_{2}$. A solution $\left(\Lambda_{1}, \Lambda_{2} ; \mathrm{M}_{1}, \mathrm{M}_{2}\right)$ is said to have height $h=\Lambda_{1}+\Lambda_{2}$. Two solutions $\left(\Lambda_{1}, \Lambda_{2} ; \mathbf{M}_{1}, M_{2}\right),\left(\Lambda_{1}^{*}, \Lambda_{2}^{*} ; M_{1}^{*}, M_{2}^{*}\right)$ are considered to be equal if $\left\{\Lambda_{1}, \Lambda_{2}\right\}=\left\{\Lambda_{1}^{*}, \Lambda_{2}^{*}\right\}$, $\left\{M_{1}, M_{2}\right\}=\left\{M_{1}^{*}, M_{2}^{*}\right\}$. Two different solutions $\left(\Lambda_{1}, \Lambda_{2} ; M_{1}, M_{2}\right),\left(\Lambda_{1}^{*}, \Lambda_{2}^{*} ; M_{1}^{*}, M_{2}^{*}\right)$ are called neighbours if $\left\{\Lambda_{1}, \Lambda_{2}\right\} \cap\left\{\Lambda_{1}^{*}, \Lambda_{2}^{*}\right\} \neq \varnothing \wedge\left\{M_{1}, M_{2}\right\} \cap\left\{M_{1}^{*}, M_{2}^{*}\right\} \neq \varnothing$.

The following lemmas are easily proved.

LEMMA 5.4. There is precisely one singular solution, namely $(1,1 ; 1,1)$; this solution has precisely one neighbouring solution $(1,5 ; 1,3)$.

LEMMA 5.5. Every non-singular solution $\left(\Lambda_{1}, \Lambda_{2} ; \mathbf{M}_{1}, \mathbf{M}_{2}\right)$ with (say) $\Lambda_{1}<\Lambda_{2}, \mathbf{M}_{1}<\mathbf{M}_{2}$, has precisely four different neighbouring solutions

$$
\left(\Lambda_{i}, \Lambda_{i j} ; \mathbf{M}_{j}, \mathbf{M}_{i j}\right), i, j \in\{1,2\}
$$

where

$$
\Lambda_{i j}=\Lambda_{i}\left(8 M_{j}^{2}-1\right)-\left(\Lambda_{1}+\Lambda_{2}\right), M_{i j}=4 \Lambda_{i} M_{j}-M_{k},
$$


with $k \in\{1,2\} \backslash\{j\}$. The corresponding heights $h=\Lambda_{1}+\Lambda_{2}, h_{i j}=\Lambda_{i}+\Lambda_{i j}$, satisfy the inequalities

$$
h_{11}<h<h_{i j}, \quad(i, j) \neq(1,1) .
$$

The following result is now obvious by Lemmas 5.4, 5.5.

THEOREM 5.2. The complete solution $\in \mathbf{N}^{4}$ of (5.4) is obtained from the singular solution $(1,1 ; 1,1)$ by successive adjunction of neighbouring solutions of bigger heights. For any solution $\left(\Lambda_{1}, \Lambda_{2} ; \mathbf{M}_{1}, \mathrm{M}_{2}\right)$, we have

$$
\operatorname{ged}\left(\Lambda_{1}, \Lambda_{2}\right)=\operatorname{ged}\left(M_{1}, M_{2}\right)=1 .
$$

The tree of solutions of (5.4) is thus of the form

$$
\begin{gathered}
(1,1 ; 1,1) \\
(1,5 ; 1,3) \\
\{\Lambda\}=\{1,5,29,65,169,349,901,985,4549,11521, \ldots\}, \\
\{M\}=\{1,3,11,17,41,59,99,153,339,571,577, \ldots\} .
\end{gathered}
$$

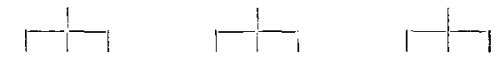

Following an idea of G. Frobenius [15], we associate to any non-singular solution $\left(\Lambda_{1}, \Lambda_{2} ; \mathbf{M}_{1}, \mathbf{M}_{2}\right)$ of $(5.4)$ with $\Lambda_{1}<\Lambda_{2}, \mathbf{M}_{1}<\mathbf{M}_{2}$ a quintuple $\left(\varepsilon ; l_{1}, l_{2} ; m_{1}, m_{2}\right)$ as follows,

$$
(1,5 ; 1,3) \mapsto(+1 ; 0,2 ; 1,1) \text {; }
$$

if

$$
\left(\Lambda_{1}, \Lambda_{2} ; \mathbf{M}_{1}, \mathbf{M}_{2}\right) \mapsto\left(\varepsilon ; l_{1}, l_{2} ; m_{1}, m_{2}\right),
$$

then for $(i, j)=(1,2),(2,1),(2,2)$,

$$
\left(\Lambda_{i}, \Lambda_{i j} ; \mathbf{M}_{j}, \mathbf{M}_{i j}\right) \mapsto\left(\varepsilon_{i j} ; l_{i}, l_{i j} ; m_{j}, m_{i j}\right),
$$

where

$$
\begin{aligned}
& \varepsilon_{12}=\varepsilon_{21}=\varepsilon, \quad \varepsilon_{22}=-\varepsilon, \\
& l_{i j}=\left(\Lambda_{i j} m_{j}-\varepsilon_{i j} \mathbf{M}_{i j}\right) / \mathbf{M}_{j}, \\
& m_{i j}=\left(\mathbf{M}_{i j} l_{i}+\varepsilon_{i j} \mathbf{M}_{j}\right) / \Lambda_{i} .
\end{aligned}
$$

By induction with respect to the tree of solutions we obtain easily 
LEMMA 5.6. Let $\left(\Lambda_{1}, \Lambda_{2} ; M_{1}, M_{2}\right)$ with $\Lambda_{1}<\Lambda_{2}, M_{1}<M_{2}$ be any non-singular solution of (5.4), and $\left(\varepsilon ; l_{1}, l_{2} ; m_{1}, m_{2}\right)$ its associated quintuple. Then $\varepsilon= \pm 1$ and $l_{1}, l_{2}, m_{1}, m_{2}$ are positive integers satisfying

(i) $\Lambda_{1} l_{2}-\Lambda_{2} l_{1}=2 \varepsilon \mathrm{M}_{1}^{2}$,

(ii) $\Lambda_{1} m_{1}-\mathrm{M}_{1} l_{1}=\varepsilon\left(4 \Lambda_{1} \mathrm{M}_{1}-\mathbf{M}_{2}\right)$,

(iii) $\Lambda_{1} m_{2}-\mathrm{M}_{2} l_{1}=\varepsilon \mathrm{M}_{1}$,

(iv) $\Lambda_{2} m_{1}-\mathrm{M}_{1} l_{2}=\varepsilon \mathrm{M}_{2}$,

(v) $\Lambda_{2} m_{2}-\mathrm{M}_{2} l_{2}=-\varepsilon \mathrm{M}_{1}$,

(vi) $\mathrm{M}_{1} m_{2}-\mathrm{M}_{2} m_{1}=-2 \varepsilon \Lambda_{1}$,

(vii) $l_{i}^{2}+1 \equiv 0\left(\bmod \Lambda_{i}\right), \quad(i=1,2)$,

(viii) $m_{i}^{2}+2 \equiv 0\left(\bmod \mathrm{M}_{i}\right), \quad(i=1,2)$,

(ix) $l_{i} \equiv 0(\bmod 2), m_{i} \equiv 1(\bmod 2), \quad(i=1,2)$,

(x) $0 \leqslant l_{i} \leqslant \Lambda_{i}, 0 \leqslant m_{i} \leqslant \mathbf{M}_{i}, \quad(i=1,2)$.

Corolla Y Any $\Lambda$ is of the form $\Lambda=x^{2}+y^{2}$ with $x$ even and $y$ odd; any $\mathrm{M}$ is of the form $\mathrm{M}=x^{2}+2 y^{2}$ with $x$ odd.

\subsection{A chain of C-minimal forms}

For any non-singular solution $\left(\Lambda_{1}, \Lambda_{2} ; M_{1}, M_{2}\right), \Lambda_{1}<\Lambda_{2}, M_{1}<M_{2}$, of (5.4) with associated quintuple $\left(\varepsilon ; l_{1}, l_{2} ; m_{1}, m_{2}\right)$, we put

$$
\lambda_{i}=\left(l_{i}^{2}+1\right) / \Lambda_{i}, \mu_{i}=\left(m_{i}^{2}+2\right) / \mathbf{M}_{i}, \quad(i=1,2) ;
$$

notice that $\lambda_{i}, \mu_{i} \in \mathrm{N}$ by Lemma 5.6 (vii) (viii). We define quadratic forms $g_{\Lambda}, h_{\mathrm{M}}\left(\Lambda=\Lambda_{i}\right.$, $\mathrm{M}=\mathrm{M}_{i}$ ) by

$$
\begin{aligned}
\Lambda g_{\Lambda}(x, y) & =\Lambda x^{2}+(4 \Lambda-2 l) x y+(\lambda-4 l) y^{2}, \\
\mathrm{M} h_{\mathrm{M}}(x, y) & =\mathrm{M} x^{2}+(4 \mathrm{M}-2 m) x y+(\mu-4 m) y^{2}
\end{aligned}
$$

Notice that by (5.6) we have the following discriminants,

$$
\delta\left(\Lambda g_{\Lambda}\right)=16 \Lambda^{2}-4, \quad \delta\left(\mathrm{M} h_{\mathrm{M}}\right)=16 \mathrm{M}^{2}-8 ;
$$

hence $g_{\Lambda}, h_{\mathrm{M}}$ are indefinite binary quadratic forms with rational coefficients and irrational roots. Notice also that by Lemma $5.6(\mathrm{x})$, the forms $g_{\Lambda}, h_{\mathrm{M}}$ satisfy the reduction condition in Lemma 5.2. 
Identically

$$
\Lambda^{2} g_{\Lambda}(x, y)=\varphi_{\Lambda}^{*}(y, z), \quad \mathbf{M}^{2} h_{\mathbf{M}}(x, y)=\psi_{\mathrm{M}}(y, w)
$$

where

$$
\varphi_{\Lambda}(y, z)=y^{2}+4 \Lambda y z+z^{2}, \psi_{M}(y, w)=2 y^{2}+4 M y w+w^{2}
$$

and

$$
z=\Lambda x-l y, \quad w=\mathrm{M} x-m y .
$$

By the chain of forms $g_{\Lambda}, h_{\mathrm{M}}$ we shall understand a tree of quadruples $\left(g_{\Lambda_{1}}, g_{\Lambda_{2}} ; h_{\mathrm{M}_{1}}\right.$, $\left.h_{\mathrm{M}_{2}}\right)$ corresponding to the tree of solutions $\left(\Lambda_{1}, \Lambda_{2} ; \mathrm{M}_{1}, \mathrm{M}_{2}\right)$ of (5.4). The simplest forms occurring in this chain are

$$
\begin{aligned}
1 g_{1} & =(1,4,1), & 1 h_{1} & =(1,2,-1), \\
5 g_{5} & =(5,16,-7), & 3 h_{3} & =(3,10,-3), \\
29 g_{29} & =(29,92,-43), & 11 h_{11} & =(11,38,-11), \\
65 g_{65} & =(65,224,-67), & 17 h_{17} & =(17,54,-25), \\
349 g_{349} & =(349,1124,-491), & 59 h_{59} & =(59,190,-83) .
\end{aligned}
$$

LEMMA 5.7. For any non-singular solution $\left(\Lambda_{1}, \Lambda_{2} ; M_{1}, M_{2}\right), \Lambda_{1}<\Lambda_{2}, M_{1}<M_{2}$, of (5.4) with associated quintuple $\left(\varepsilon ; l_{1}, l_{2} ; m_{1}, m_{2}\right)$, we have

(i) $g_{\Lambda_{2}}\left(l_{2}, \Lambda_{2}\right)=g_{\Lambda_{2}}\left(l_{2}-4 \Lambda_{2}, \Lambda_{2}\right)=1$ for any $\varepsilon$,

(iia) $g_{\Lambda_{2}}\left(m_{2}, \mathbf{M}_{2}\right)=g_{\Lambda_{2}}\left(m_{1}-4 \mathbf{M}_{1}, \mathbf{M}_{1}\right)=-2$ if $\varepsilon=+1$,

(iib) $g_{\Lambda_{2}}\left(m_{2}-4 \mathrm{M}_{2}, \mathrm{M}_{2}\right)=g_{\Lambda_{2}}\left(m_{1}, \mathrm{M}_{1}\right)=-2$ if $\varepsilon=-1$,

(iii) $h_{\mathrm{M}_{2}}\left(m_{2}, \mathrm{M}_{2}\right)=h_{\mathrm{M}_{2}}\left(m_{2}-4 \mathrm{M}_{2}, \mathrm{M}_{2}\right)=2$ for any $\varepsilon$,

(iva) $h_{\mathrm{M}_{2}}\left(l_{1}, \Lambda_{1}\right)=-1, h_{\mathrm{M}_{2}}\left(m_{1}-4 \mathrm{M}_{1}, \mathrm{M}_{1}\right)=-2$ if $\varepsilon=+1$,

(ivb) $h_{\mathrm{M}_{2}}\left(l_{1}-4 \Lambda_{1}, \Lambda_{1}\right)=-1, h_{\mathrm{M}_{2}}\left(m_{1}, \mathrm{M}_{1}\right)=-2$ if $\varepsilon=-1$.

Proof. Analogous to that of Lemma 8, chapter II of [4].

COROLLAR Y. Let $f(x, y)=x^{2}+\beta x y+\gamma y^{2}$ and suppose that $f$ satisfies some of the following inequalities,

(i) $f\left(l_{2}, \Lambda_{2}\right) \geqslant 1, f\left(l_{2}-4 \Lambda_{2}, \Lambda_{2}\right) \geqslant 1$,

(iia) $f\left(m_{2}, \mathrm{M}_{2}\right) \leqslant-2, f\left(m_{1}-4 \mathrm{M}_{1}, \mathrm{M}_{1}\right) \leqslant-2$,

(iib) $f\left(m_{2}-4 \mathrm{M}_{2}, \mathrm{M}_{2}\right) \leqslant-2, f\left(m_{1}, \mathrm{M}_{1}\right) \leqslant-2$,

(iii) $f\left(m_{2}, \mathbf{M}_{2}\right) \geqslant 2, f\left(m_{2}-4 \mathbf{M}_{2}, \mathbf{M}_{2}\right) \geqslant 2$,

(iva) $f\left(l_{1}, \Lambda_{1}\right) \leqslant-1, f\left(m_{1}-4 \mathrm{M}_{1}, \mathrm{M}_{1}\right) \leqslant-2$,

(ivb) $f\left(l_{1}-4 \Lambda_{1}, \Lambda_{1}\right) \leqslant-1, f\left(m_{1}, M_{1}\right) \leqslant-2$. 
Then the following holds,

$$
\begin{aligned}
& f=g_{\Lambda_{2}} \quad \text { in case } \varepsilon=+1 \text { and } f \text { satisfies (i), (iia), } \\
& f=g_{\Lambda_{2}} \quad \text { in case } \varepsilon=-1 \text { and } f \text { satisfies (i), (iib), } \\
& f=h_{\mathrm{M}_{2}} \quad \text { in case } \varepsilon=+1 \text { and } f \text { satisfies (iii), (iva), } \\
& f=h_{\mathrm{M}_{2}} \quad \text { in case } \varepsilon=-1 \text { and } f \text { satisfies (iii), (ivb). }
\end{aligned}
$$

Proof. Analogous to that of Lemma 8, Corollary, chapter II of [4].

LEм М 5.8. For any form $g_{\Lambda}$, we have $g_{\Lambda}^{*} \approx-g_{\Lambda}$; for any form $h_{\mathrm{M}}$, we have $h_{\mathrm{M}} \approx-h_{\mathrm{M}}$.

Proof. Using Lemmas 5.1, 5.6, 5.7, the proof is analogous to that of Lemma 9, chapter II of [4].

CoROLLARY. For any form $g_{\Lambda}$, we have $\left(\vartheta_{1}\left(g_{\Lambda}\right)\right)^{*} \approx \vartheta_{2}\left(g_{\Lambda}\right)$; for any form $h_{\mathrm{M}}$, we have $\vartheta_{1}\left(h_{\mathrm{M}}\right) \approx \vartheta_{2}\left(h_{\mathrm{M}}\right)$.

Proof. This follows from Lemma 5.8 together with (5.1), (5.2), (5.3).

L Емм 5.9. For any forms $g_{\Lambda}, h_{\mathrm{M}}$, we have

$$
\begin{array}{ll}
\left|g_{\Lambda}(x, y)\right| \geqslant 2 & \text { for all }(x, y) \in \mathbf{Z}_{0}^{2} \\
\left|g_{\Lambda}(x, y)\right| \geqslant 1 & \text { for all }(x, y) \in \mathbf{Z}_{1}^{2} \\
\left|h_{\mathrm{M}}(x, y)\right| \geqslant 2 & \text { for all }(x, y) \in \mathbf{Z}_{0}^{2} \\
\left|h_{\mathrm{M}}(x, y)\right| \geqslant 1 & \text { for all }(x, y) \in \mathbf{Z}_{1}^{2} .
\end{array}
$$

Proof. Analogous to that of Lemma 10, chapter II of [4].

Corolla RY 1. For any forms $g_{\Lambda}, h_{\mathrm{M}}$, we have

$$
\begin{aligned}
& v_{0}\left(g_{\Lambda}\right)=2, v_{1}\left(g_{\Lambda}\right)=1, v\left(g_{\Lambda}\right)=2, \\
& v_{0}\left(h_{\mathrm{M}}\right)=2, v_{1}\left(h_{\mathrm{M}}\right)=1, v\left(h_{\mathrm{M}}\right)=2 .
\end{aligned}
$$

CoR OLlaRy 2. All forms $g_{\Lambda}, h_{\mathrm{M}}$ satisfy condition $\left(^{*}\right)$ of Lemma $5.3 \mathrm{C}$, hence Lemma $5.3 \mathrm{C}$ and Theorem 5.1 can be applied.

Proof. Both corollaries follow from Lemmas 5.7, 5.9. 4-752903 Acta mathematica 134. Imprimé le 30 Juillet 1975 
LEMM 4 5.10. Let $f(x, y)=x^{2}+\beta x y+\gamma y^{2} ;$ then (with the notation of Lemma 5.7, Corollary)

(1a) $\delta(f) \geqslant 16-4 / \Lambda_{2}^{2}=\delta\left(g_{\Lambda_{z}}\right)$ if $\varepsilon=+1$ and (iia) holds,

(lb) $\delta(f) \geqslant 16-4 / \Lambda_{2}^{2}=\delta\left(g_{\Lambda_{2}}\right)$ if $\varepsilon=-1$ and (iib) holds,

(2a) $\delta(f) \geqslant 16-8 / \mathrm{M}_{2}^{2}=\delta\left(h_{\mathrm{M}_{2}}\right)$ if $\varepsilon=+1$ and (iva) holds,

(2b) $\delta(f) \geqslant 16-8 / \mathrm{M}_{2}^{2}=\delta\left(h_{\mathrm{M}_{2}}\right) \quad$ if $\varepsilon=-1$ and (ivb) holds.

Proof. Analogous to that of Lemma 11, chapter II of [4].

LFMMA 5.11. Let $f(x, y)=x^{2}+\beta x y+\gamma y^{2} ;$ then

$$
\begin{aligned}
& \delta(f) \geqslant 16+4 / \Lambda_{2}^{2} \quad \text { if } f\left(l_{2}, \Lambda_{2}\right) \leqslant-1, f\left(l_{2}-4 \Lambda_{2}, \Lambda_{2}\right) \leqslant-1 \\
& \delta(f) \geqslant 16+8 / M_{2}^{2} \quad \text { if } f\left(m_{2}, M_{2}\right) \leqslant-2, f\left(m_{2}-4 M_{2}, M_{2}\right) \leqslant-2 .
\end{aligned}
$$

Proof. Analogous to that of Lemma 12, chapter II of [4].

LFMм A 5.12. Let $f(x, y)=x^{2}+\beta x y+\gamma y^{2}$, where $2 \leqslant \beta \leqslant 4 \quad$ and $0<\delta(f)=\beta^{2}-4 \gamma<16$.

Suppose that

$$
\begin{array}{ll}
|f(x, y)| \geqslant 2 & \text { for all }(x, y) \in \mathbf{Z}_{0}^{2} \\
|f(x, y)| \geqslant 1 & \text { for all }(x, y) \in \mathbf{Z}_{1}^{2} .
\end{array}
$$

Then $f$ is either $a g_{\Lambda}$ or $a h_{\mathrm{M}}$.

Proof. We shall use Cassels' notation,

$$
P(x, y): f(x, y)>0, N(x, y): f(x, y)<0 .
$$

If $P(-1,1)$, then $1-\beta+\gamma \geqslant 2$, a contradiction to $2 \leqslant \beta \leqslant 4$ and $\beta^{2}-4 \gamma>0$. Hence $N(-1,1)$, i.e. $1-\beta+\gamma \leqslant-2$ or

$$
\beta \geqslant \gamma+3
$$

If $P(0,1)$, then $\gamma \geqslant 1$, which together with (5.11) gives $\beta \geqslant 4$. Since $2 \leqslant \beta \leqslant 4$, we must have $\beta=4$ and consequently $\gamma=1$; hence $f=(1,4,1)=g_{1}$.

In the sequel we assume that $f \neq g_{1}$, hence $N(0,1)$, i.e.

$$
\gamma \leqslant-1 \text {. }
$$

If $P(-3,1)$, then $9-3 \beta+\gamma \geqslant 2$, which together with (5.12) gives $\beta \leqslant 2$. Since $2 \leqslant \beta \leqslant 4$, we must have $\beta=2$ and consequently $\gamma=-1$; hence $f=(1,2,-1)=h_{1}$. 
In the sequel we assume that $f \neq g_{1}, h_{1}$, hence

$$
N(0,1) \text { and } N(-3,1) \text {. }
$$

The proof now follows by induction. Let $\left(\Lambda_{1}, \Lambda_{2} ; M_{1}, M_{2}\right), \Lambda_{1}<\Lambda_{2}, M_{1}<M_{2}$, be any non-singular solution of (5.4) with associated quintuple $\left(\varepsilon, l_{1}, l_{2} ; m_{1}, m_{2}\right)$, and suppose that we have

$$
\begin{aligned}
& N\left(l_{1}, \Lambda_{1}\right) \text { and } N\left(m_{1}-4 \mathrm{M}_{1}, \mathrm{M}_{1}\right) \quad \text { in case } \varepsilon=+1, \\
& N\left(l_{1}-4 \Lambda_{1}, \Lambda_{1}\right) \text { and } N\left(m_{1}, \mathrm{M}_{1}\right) \quad \text { in case } \varepsilon=-1
\end{aligned}
$$

Notice that the inductive hypothesis for the solution $(1,5 ; 1,3)$ of $(5.4)$, which has $\varepsilon=+1$, is precisely (5.13), and thus is satisfied if $f \neq g_{1}, h_{1}$.

From the inductive hypothesis for $\left(\Lambda_{1}, \Lambda_{2} ; M_{1}, M_{2}\right)$ we want to deduce that $f=g_{\Lambda_{2}}$ or $h_{\mathrm{M}_{2}}$ or to prove the inductive hypothesis for at least one of the three neighbouring solutions of $\left(\Lambda_{1}, \Lambda_{2} ; M_{1}, M_{2}\right)$ of bigger height. By Lemma 5.5 these solutions are of the form

$$
\begin{aligned}
& \left(\Lambda_{1},-; \mathrm{M}_{2},-\right) \text { with quintuple }\left(\varepsilon ; l_{1},-; m_{2},-\right), \\
& \left(\Lambda_{2},-; \mathrm{M}_{1},-\right) \text { with quintuple }\left(\varepsilon ; l_{2},-; m_{1},-\right), \\
& \left(\Lambda_{2},-; \mathrm{M}_{2},-\right) \quad \text { with quintuple }\left(-\varepsilon ; l_{2},-; m_{2},-\right) .
\end{aligned}
$$

If $P\left(m_{2}, \mathrm{M}_{2}\right)$ and $P\left(m_{2}-4 \mathrm{M}_{2}, \mathrm{M}_{2}\right)$, then $f=h_{\mathrm{M}_{2}}$ by Lemma 5.7, Corollary, since either (iii) (iva) or (iii) (ivb) are valid.

Otherwise, either $N\left(m_{2}, \mathrm{M}_{2}\right)$ or $N\left(m_{2}-4 \mathrm{M}_{2}, \mathrm{M}_{2}\right)$, in which case we distinguish between four subcases:

(1) $\varepsilon=+1$ and $N\left(m_{2}, \mathrm{M}_{2}\right)$. If $P\left(l_{2}, \Lambda_{2}\right)$ and $P\left(l_{2}-4 \Lambda_{2}, \Lambda_{2}\right)$, then $f=g_{\mathrm{M}_{2}}$ by Lemma 5.7 , Corollary, since (i) (iia) are valid. Otherwise either $N\left(l_{2}, \Lambda_{2}\right)$ or $N\left(l_{2}-4 \Lambda_{2}, \Lambda_{2}\right)$; in case $N\left(l_{2}, \Lambda_{2}\right)$ the inductive hypothesis (5.14a) is satisfied for $\left(\Lambda_{2},-; M_{1},-\right)$, in case $N\left(l_{2}-4 \Lambda_{2}\right.$, $\left.\Lambda_{2}\right)$ the inductive hypothesis $(5.14 \mathrm{~b})$ is satisfied for $\left(\Lambda_{2},-; \mathrm{M}_{2},-\right)$.

(2) $\varepsilon=+1$ and $N\left(m_{2}-4 \mathrm{M}_{2}, \mathrm{M}_{2}\right)$. Then the inductive hypothesis (5.14a) is satisfied for $\left(\Lambda_{1},-; M_{2},-\right)$.

(3) $\varepsilon=-1$ and $N\left(m_{2}, \mathrm{M}_{2}\right)$. Then the inductive hypothesis $(5.14 \mathrm{~b})$ is satisfied for $\left(\Lambda_{1},-; M_{2},-\right)$.

(4) $\varepsilon=-1$ and $N\left(m_{2}-4 \mathrm{M}_{2}, \mathrm{M}_{2}\right)$. If $P\left(l_{2}, \Lambda_{2}\right)$ and $P\left(l_{2}-4 \Lambda_{2}, \Lambda_{2}\right)$, then $f=g_{\Lambda_{2}}$ by Lemma 5.7, Corollary, since (i) (iib) are valid. Otherwise either $N\left(l_{2}, \Lambda_{2}\right)$ or $N\left(l_{2}-4 \Lambda_{2}, \Lambda_{2}\right)$; in case $N\left(l_{2}, \Lambda_{2}\right)$ the inductive hypothesis (5.14a) is satisfied for $\left(\Lambda_{2},-; \mathrm{M}_{2},-\right)$, in case $N\left(l_{2}-4 \Lambda_{2}\right.$, $\left.\Lambda_{2}\right)$ the inductive hypothesis $(5.14 b)$ is satisfied for $\left(\Lambda_{2},-; M_{1},-\right)$.

Consequently, if $f$ were not a $g_{\Lambda}$ or a $h_{\mathrm{M}}$, it would have to satisfy either (5.14a) or 
(5.14b) for an infinite sequence $\left(\Lambda_{1}^{(r)}, \Lambda_{2}^{(r)} ; \mathbf{M}_{1}^{(r)}, \mathbf{M}_{2}^{(r)}\right), r \in \mathbf{N}$, with heights $h^{(r)} \rightarrow \infty$ for $r \rightarrow \infty$. It would then follow from Lemma $5.10((2 a)$ or $(2 b)$ apply) that

$$
\delta(f) \geqslant 16-8 / \mathbf{M}_{2}^{(r)} \text { for } r \in \mathbf{N} .
$$

However, since $\mathrm{M}_{2}^{(r)} \rightarrow \infty$ for $r \rightarrow \infty$, this would imply that $\delta(f) \geqslant \mathrm{I} 6$, a contradiction. This proves the lemma.

LEMм А 5.13. There are $2^{\mathbb{N}_{0}}$ different forms

$$
\begin{aligned}
& f(x, y)=x^{2}+\beta x y+\gamma y^{2} \quad \text { with } \\
& 2 \leqslant \beta \leqslant 4, \delta(f)=\beta^{2}-4 \gamma=16, v(f)=2 .
\end{aligned}
$$

Proof. Analogous to that of Lemma 14, chapter II of [4].

\subsection{The main theorem on the C-minimum of forms}

THEOREM 5.3. Let $f(x, y)=\alpha x^{2}+\beta x y+\gamma y^{2}, \delta(f)>0$.

A. If

$$
\sqrt{\delta(f)} / v(f)<2
$$

then $f$ is C-equivalent to a multiple of some $g_{\Lambda}$ or $h_{\mathrm{M}}$.

B. Conversely (5.15) holds for all forms C-equivalent to a multiple of some $g_{\Lambda}$ or $h_{\mathrm{M}}$; specifically

$$
\sqrt{\delta\left(g_{\Lambda}\right)} / \nu\left(g_{\Lambda}\right)=\sqrt{4-1 / \Lambda^{2}}, \quad \sqrt{\delta\left(h_{\mathrm{M}}\right)} / \nu\left(h_{\mathrm{M}}\right)=\sqrt{4-2 / \mathrm{M}^{2}} .
$$

C. There are $2^{\aleph_{0}}$ forms $f$, none of which are C-equivalent to a multiple of any other, such that

$$
\sqrt{\delta(f)} / v(f)=2
$$

Proof. Part B follows by Lemma 5.1, (5.7) and Lemma 5.9, Corollary 1.

Part $\mathrm{C}$ follows from Lemma 5.13, since any $C$-equivalence class of forms is denumerable.

To prove part A we notice, that by Lemma 5.1 we may as well assume that

$$
0<\delta(f)<16, \quad \nu(f)=2,
$$

hence by the definition of $C$-minimum either $v_{1}(f)=1$ or $v_{0}(f)=2$.

Case 1. $v_{1}(f)=1$. Then for any $\varepsilon>0$, there are $(a, c) \in \mathbf{Z}_{1}^{2}$ such that

$$
1=v_{1}(f) \leqslant|f(a, c)|=\alpha^{\prime}<1+\varepsilon .
$$


Hence by Lemma 5.2 we have

$$
\pm f \approx f^{\prime}=\left(\alpha^{\prime}, \beta^{\prime}, \gamma^{\prime}\right)
$$

where

$$
1 \leqslant \alpha^{\prime}<1+\varepsilon, \quad 2 \alpha^{\prime} \leqslant \beta^{\prime} \leqslant 4 \alpha^{\prime}, \delta\left(f^{\prime}\right)=\delta(f) .
$$

If $\alpha^{\prime}=1$, then $f^{\prime}$ is some $g_{\Lambda}, h_{\mathrm{M}}$ by Lemma 5.12, and the conclusion follows from (5.17). Otherwise we can find an infinite sequence of forms

$$
f_{n}=\left(\alpha_{n}, \beta_{n}, \gamma_{n}\right), \quad n \in \mathbf{N},
$$

with

$$
\lim \alpha_{n}=1,2 \alpha_{n} \leqslant \beta_{n} \leqslant 4 \alpha_{n}, \delta\left(f_{n}\right)=\delta(f),
$$

each $f_{n}$ being $C$-equivalent to $\pm f$. By a simple compactness argument, we may as well assume that

$$
\beta_{n} \rightarrow \beta_{0}, \gamma_{n} \rightarrow \gamma_{0} \quad \text { for } n \rightarrow \infty \text {. }
$$

Then

$$
f_{0}=\left(1, \beta_{0}, \gamma_{0}\right)
$$

has

$$
2 \leqslant \beta_{0} \leqslant 4, \delta\left(f_{0}\right)=\delta(f)
$$

Further since

$$
\left|f_{0}(x, y)\right|=\lim _{n \rightarrow \infty}\left|f_{n}(x, y)\right|
$$

we have

$$
\begin{array}{ll}
\left|f_{0}(x, y)\right| \geqslant 1 & \text { for all }(x, y) \in \mathbf{Z}_{1}^{2}, \\
\left|f_{0}(x, y)\right| \geqslant 2 & \text { for all }(x, y) \in \mathbf{Z}_{0}^{2} .
\end{array}
$$

Hence by Lemma 5.12, $f_{0}$ is some $g_{\Lambda}, h_{\mathrm{M}}$. However, by Lemma 5.9, Corollary 2, we may apply Theorem 5.1 to $f_{0}\left(=g_{\Lambda}\right.$ or $\left.h_{\mathrm{M}}\right)$, and consequently for some sufficiently large $n$, $f_{n}=a f_{0}\left(=a g_{\Lambda}\right.$ or $\left.a h_{\mathrm{M}}\right)$. Since also $f \approx \pm f_{n}$, this proves $\mathrm{A}$ in this case.

Case $2 . v_{0}(f)=2$. Then by Lemma 5.1,

$$
\nu_{1}\left(f^{*}\right)=1, v\left(f^{*}\right)=2,0<\delta\left(f^{*}\right)=\delta(f)<16,
$$

and hence by case 1 , we have $f^{*} \approx a g_{\Lambda}$ or $a h_{M} ;$ also $a= \pm 1$, since

$$
\nu_{1}\left(a g_{\Lambda}\right)=\nu_{1}\left(a h_{\mathrm{M}}\right)=|a|
$$


by Lemma 5.1 and Lemma 5.9, Corollary 1. Finally

$$
2 \nu_{1}(f)=\nu_{0}\left(f^{*}\right)=\nu_{0}\left( \pm g_{\Lambda}\right)=\nu_{0}\left( \pm h_{M}\right)=2 \text {, }
$$

and thus also $v_{1}(f)=1$, and hence $f$ belongs to case 1 also. This ends the proof of part $A$.

\subsection{The main theorem on the C-approximation constant}

THEоR EM 5.4. Let $\vartheta \in \mathbf{R} \backslash \mathbf{Q}$.

A. If

$$
d(\vartheta)<2,
$$

then $\vartheta$ is C-equivalent to a root in some $g_{\Lambda}$ or $h_{\mathrm{M}}$.

B. Conversely (5.18) holds if $\vartheta$ is C-equivalent to a root in some $g_{\Lambda}$ or $h_{\mathrm{M}}$; specifically

$$
\begin{aligned}
& d(\vartheta)=\sqrt{4-1 / \Lambda^{2}}, \quad \text { when } g_{\Lambda}(\vartheta, 1)=0 \\
& d(\vartheta)=\sqrt{4-2 / M^{2}}, \quad \text { when } h_{\mathrm{M}}(\vartheta, 1)=0
\end{aligned}
$$

For any $g_{\Lambda}$, we have $\left(\vartheta_{1}\left(g_{\Lambda}\right)\right)^{*} \approx \vartheta_{2}\left(g_{\Lambda}\right)$; for any $h_{\mathrm{M}}$, we have $\vartheta_{1}\left(h_{\mathrm{M}}\right) \approx \vartheta_{2}\left(h_{\mathrm{M}}\right)$.

C. There are $2^{\aleph_{0}}$ different $C$-equivalence classes of irrationals $\vartheta$, such that $d(\vartheta)=2$ !

Proof. To prove part A notice that since $d(\vartheta)=\max \left(d_{0}(\vartheta), \frac{1}{2} d_{1}(\vartheta)\right)$, either $d_{0}(\vartheta)=d(\vartheta)<2$ or $d_{1}(\vartheta)=2 d(\vartheta)<4$

Case 1. $d_{1}(\vartheta)=2 d(\vartheta)<4$. We consider the form

$$
f(x, y)=2 d(\vartheta) x(\vartheta x-y) .
$$

By the definition of $d(\vartheta): \forall \varepsilon>0 \exists Y_{0}=Y_{0}(\varepsilon)>0$, such that

$$
\begin{array}{lll}
|f(x, y)|>2-\varepsilon & \text { for all }(x, y) \in \mathbf{Z}_{0}^{2} & \text { with }|\vartheta x-y|<Y_{0}(\varepsilon), \\
|f(x, y)|>1-\varepsilon & \text { for all }(x, y) \in \mathbf{Z}_{1}^{2} & \text { with }|\vartheta x-y|<Y_{0}(\varepsilon)
\end{array}
$$

Further, since $d_{1}(\vartheta)=2 d(\vartheta)$, there is a sequence $\left(a_{n}, c_{n}\right) \in \mathbf{Z}_{1}^{2}$ such that

$$
\left|f\left(a_{n}, c_{n}\right)\right| \rightarrow 1, a_{n} \rightarrow \infty,\left|\vartheta a_{n}-c_{n}\right| \rightarrow 0 \text { for } n \rightarrow \infty .
$$

It is now clear that the proof of Theorem III, chapter II of [4] carries over with obvious modifications; this gives the conclusion in case 1 .

Case 2. $d_{0}(\vartheta)=d(\vartheta)<2$. Then by Theorem 4.11, Corollary,

$$
d_{1}\left(\vartheta^{*}\right)=2 d_{0}(\vartheta)=2 d(\vartheta)=2 d\left(\vartheta^{*}\right)<4
$$


hence by case $1, \vartheta^{*} \approx \vartheta^{\prime}$, where $\vartheta^{\prime}$ is a root of some $g_{\Lambda}$ or $h_{\mathrm{M}}$. But then by Theorem 4.11, Corollary, Lemma 5.1, Lemma 5.3 B, Lemma 5.9, Corollary,

$$
d_{1}(\vartheta)=2 d_{0}\left(\vartheta^{*}\right)=2 d_{0}\left(\vartheta^{\prime}\right)=2 d\left(\vartheta^{\prime}\right)=2 d\left(\vartheta^{*}\right)=2 d(\vartheta),
$$

and thus $\vartheta$ belongs to case 1 also. This ends the proof of part $A$.

Part B follows directly from Theorem 5.3 B, Lemma 3 B and Lemma 5.8, Corollary.

Proof of C. By Theorem 5.3 C together with Lemma 5.3 A, there are $2^{x_{0}}$ different $C$. equivalence classes of irrationals $\vartheta$ such that $d(\vartheta) \leqslant 2$. By Theorem $5.4 \mathrm{~A}$ there are only enumerably many different $C$-equivalence classes of irrationals $\vartheta$ such that $d(\vartheta)<2$.

\subsection{Other methods}

Instead of the proof of Theorems 5.3, 5.4 given above, which follows closely the proof of J. W. S. Cassels [4] for the Markoff chain, we could have extended either (1) the proof of A. Markoff [24] as presented by L. E. Dickson [9] or (2) the proof of C. G. Lekkerkerker [21].

The extension of Markoff's method is based on the formula for $d(\vartheta)$ in Theorem 4.11, and a similar formula for $v(f)$ obtained by developing a theory of $C$-reduced and $C$-dually reduced quadratic forms.

Lekkerkerker's method is extended as follows: For any basis $\left(\mathbf{e}_{1}, \mathbf{e}_{2}\right)$ of $\mathbf{R}^{2}$ over $\mathbf{R}$, let

$$
\begin{gathered}
L=L\left(\mathbf{e}_{1}, \mathbf{e}_{2}\right)=\left\{x_{1} \mathbf{e}_{1}+x_{2} \mathbf{\theta}_{2} \mid x_{1}, x_{2} \in \mathbf{Z}\right\}, \\
L_{j}=L_{j}\left(\mathbf{e}_{1}, \mathbf{e}_{2}\right)=\left\{x_{1} \mathbf{e}_{1}+x_{2} \mathbf{e}_{2} \mid\left(x_{1}, x_{2}\right) \in \mathbf{Z}_{j}^{2}\right\}, \quad(j=0,1) .
\end{gathered}
$$

For

$$
S_{0}=\left\{\left(\xi_{1}, \xi_{2}\right)|| \xi_{1} \xi_{2} \mid<1\right\}
$$

$L$ is called $C$-admissible for $S_{0}$ if

$$
L_{0} \cap S_{0}=L_{1} \cap \frac{1}{\sqrt{2}} S_{0}=\varnothing
$$

For example

$$
\begin{gathered}
L((1 / \sqrt{2}, 1 / \sqrt{2}),(-1+1 / \sqrt{2}, 1+1 / \sqrt{2})) \quad \text { with } \operatorname{det} L=\sqrt{2} \\
L((1 / \sqrt{2}, 1 / \sqrt{2}),(-\sqrt{3 / 2}, \sqrt{3} / 2)) \quad \text { with } \operatorname{det} L=\sqrt{3}
\end{gathered}
$$

are both $C$-admissible for $S_{0}$.

Essentially all $C$-admissible $L$ 's for $S_{0}$ of $\operatorname{det} L<2$ are constructed by the procedure of Lekkerkerker, however using the matrices

$$
C_{0}=\left(\begin{array}{ll}
1 & 3 \\
1 & 1
\end{array}\right), D_{0}=\left(\begin{array}{ll}
3 & 1 \\
1 & 1
\end{array}\right), \hat{D}_{0}=\left(\begin{array}{ll}
1 & 1 \\
1 & 3
\end{array}\right),
$$


which satisfy the relations

$$
C_{0} D_{0}=\hat{D}_{0} K C_{0}, \hat{D}_{0} C_{0}=C_{0} K D_{0}, C_{0} \hat{D}_{0}=D_{0} C_{0},
$$

where

$$
K=\left(\begin{array}{rr}
-1 & 8 \\
0 & -1
\end{array}\right)
$$

For example

$$
\begin{gathered}
A=D_{0} C_{0} \hat{D}_{0} C_{0}=4\left(\begin{array}{cc}
12 & 19 \\
5 & 8
\end{array}\right), \\
B=D_{0} C_{0}^{2}=2\left(\begin{array}{rr}
7 & 11 \\
3 & 5
\end{array}\right),
\end{gathered}
$$

and hence we have the following associated forms,

$$
\begin{aligned}
& f_{\mathrm{C}_{0}}=(1,1-1,-3)=(1,0,-3) \approx g_{1}, \\
& \left.f_{\mathrm{D}_{0}}=1,1-3,-1\right)=(1,-2,-1) \approx h_{1}, \\
& f_{A}=(5,8-12,-19)=(5,-4,-19) \approx 5 g_{5}, \\
& f_{B}=(3,5-7,-11)=(3,-2,-11) \approx 3 h_{3} .
\end{aligned}
$$

I shall confine myself with this hint of the extension of Lekkerkerker's method.

Also recent work of $\mathrm{H}$. Cohn [6], [7] and M. Hall [17] on the Markoff chain have extensions to the present situation.

\section{Chapter 6}

\section{Complex binary quadratic forms}

\subsection{Reduction theory}

We consider binary quadratic forms $\Phi=(A, B, C): \mathbf{C}^{2} \rightarrow \mathbf{C}$, given by

$$
\Phi:(X, Y) \mapsto A X^{2}+B X Y+C Y^{2},
$$

where $A, B, C \in \mathbf{C}$ satisfy the following condition,

$$
A \neq 0, D=B^{2}-4 A C \neq 0, \quad \xi, \eta \in \mathbf{C} \backslash \mathbf{Q}(i) .
$$

Here $\xi, \eta$ are the roots of $\Phi$, i.e. of $\Phi(x, 1)=0$.

For any complex unimodular matrix $M$, we let $\tilde{M}:(X, Y) \mapsto(a X+b Y, c X+d Y)$ be the corresponding linear map, and (as usual) $m$ be the corresponding homographic map. 
Notice that if $\Phi$ satisfies (6.1), then also any $\Psi$ equivalent to $\Phi$, i.e. of the form $\Psi=\Phi \circ \tilde{M}$ where $M$ is unimodular. In fact, this follows from the formulae,

$$
\begin{gathered}
D_{\Psi}=(\operatorname{det} M)^{2} D_{\Phi}= \pm D_{\Phi}, \\
\xi_{\Psi}=m^{-1}\left(\xi_{\Phi}\right), \eta_{\Psi}=m^{-1}\left(\eta_{\Phi}\right) \\
A_{\Psi}=\Phi(a, c), C_{\Psi}=\Phi(b, d), A_{\Psi}+B_{\Psi}+C_{\Psi}=\Phi(a+b, c+d) .
\end{gathered}
$$

Notice also that an integral form $(A, B, C)$, i.e. a form with $A, B, C \in \mathbf{Z}[i]$, satisfies (6.1) precisely when $D=B^{2}-4 A C$ is not a square in $\mathbf{Z}[i]$.

Definition 6.1. A binary quadratic form satisfying (6.1) is called reduced if (with suitable notation)

$$
\xi \in \mathcal{J} \text { and } \bar{\eta} \in \mathcal{J}^{*} \text {, }
$$

and is called dually reduced if (with suitable notation)

$$
\xi \in \mathfrak{J}^{*} \text { and } \bar{\eta} \in \mathfrak{J} \text {. }
$$

Notice that if $(A, B, C)$ is reduced (dually reduced), then $(\bar{A}, \bar{B}, \bar{C})$ is dually reduced (reduced), $(C, B, A)$ is dnally reduced (reduced) and $(\bar{C}, \vec{B}, \bar{A})$ is reduced (dually reduced).

Notice also that if $\Phi$ is reduced (dually reduced), then $\lambda \Phi, \lambda \in \mathbf{C} \backslash\{0\}$, is reduced (dually reduced) and $\Phi \circ \tilde{S}, \Phi \circ \tilde{S}^{-1}$ are both reduced (dually reduced), the last statement being a consequence of Lemma 1.1 (viii) (ix).

THeоRem 6.1. Suppose that $\Phi=(A, B, C)$ satisfying (6.1) represents primitively a number $A^{\prime}$ with

$$
0<\left|A^{\prime}\right| \leqslant \sqrt{|\bar{D}| / 2}
$$

Then there exists a form $\Phi^{\prime}=\left(A^{\prime}, B^{\prime}, C^{\prime}\right)$, which is equivalent to $\Phi$, and such that $\Phi^{\prime}$ is either reduced or dually reduced.

Proof. By a suitable choice between the two values of $\sqrt{D}$ and $\sqrt{-D}$ we can make sure that either

(a) $\arg \left(\sqrt{D} / A^{\prime}\right) \in[\pi / 4,3 \pi / 4]$

or

(b) $\arg \left(\sqrt{-D} / A^{\prime}\right) \in[\pi / 4,3 \pi / 4]$.

By assumption there exist $a_{0}, c_{0} \in \mathbf{Z}[i]$ with ged $\left(a_{0}, c_{0}\right)=1$ and $\Phi\left(a_{0}, c_{0}\right)=A^{\prime}$. Then determine $b_{0}, d_{0} \in \mathbf{Z}[i]$, such that $a_{0} d_{0}-b_{0} c_{0}=1$ in case (a) and $a_{0} d_{0}-b_{0} c_{0}=i$ in case (b), and put

$$
M_{0}=\left(\begin{array}{cc}
a_{0} & b_{0} \\
c_{0} & d_{0}
\end{array}\right), M_{k}=M_{0}\left(\begin{array}{ll}
1 & k \\
0 & 1
\end{array}\right), k \in \mathbf{Z}[i]
$$


In either case the forms

$$
\Phi_{k}=\Phi \circ \tilde{M}_{k}, \quad k \in \mathbf{Z}[i],
$$

are equivalent to $\Phi$, and $\Phi_{k}(1,0)=\Phi\left(a_{0}, c_{0}\right)=A^{\prime}$; hence we need only prove that $\Phi_{k}$ is reduced or dually reduced for a suitable $k \in \mathbb{Z}[i]$.

However, the roots $\xi_{k}, \eta_{k}$ (suitably enumerated) of $\Phi_{k}$ satisfy the following relations,

$$
\begin{aligned}
& \xi_{k}=\xi_{0}-k, \eta_{k}=\eta_{0}-k \text { for all } k \in \mathbf{Z}[i], \\
& \xi_{k}-\eta_{k}=\sqrt{D} / A^{\prime} \quad \text { in case (a) for all } k \in \mathbf{Z}[i], \\
& \xi_{k}-\eta_{k}=\sqrt{-D} / A^{\prime} \quad \text { in case (b) for all } k \in \mathbf{Z}[i] .
\end{aligned}
$$

Hence it follows from $(6.6 \mathrm{a}),(6.6 \mathrm{~b})$ that

$$
\arg \left(\xi_{k}-\eta_{k}\right) \in[\pi / 4,3 \pi / 4] \text { for all } k \in \mathbf{Z}[i] \text {. }
$$

Also, it follows from (6.6a), (6.6b) and the assumption of the theorem, that

$$
\left|\xi_{k}-\eta_{k}\right| \geqslant \sqrt{2} \text { for all } k \in \mathbf{Z}[i] \text {. }
$$

By (6.5) we choose $k \in \mathbf{Z}[i]$, such that

$$
\eta_{k} \in[0,1] \times[0,-i]
$$

If

$$
\eta_{k} \in[0,1] \times\left[-\frac{i}{2},-i\right] \subseteq \mathfrak{J}^{*}
$$

then by (6.7), (6.8), $\xi_{k} \in \mathcal{J}$, and $\Phi_{k}$ is reduced. If

$$
\eta_{k} \in[0,1] \times\left[0,-\frac{i}{2}\right] \subseteq \overline{\mathfrak{J}}
$$

then by the same argument, either $\Phi_{k}$ or $\Phi_{k-1}$ or $\Phi_{k+1}$ is dually reduced.

COROLLARY Every form $\Phi$ satisfying (6.1) is equivalent to a reduced form and also equivalent to a dually reduced form.

Proof. The result follows from Theorem 6.1 together with a well-known result of O. Perron [26], that every form satisfying (6.1) will represent primitively (in the Gaussian case) a number $A^{\prime}$ with $0<\left|A^{\prime}\right| \leqslant \sqrt{|D| / 3}$, and finally the remark, that if $(A, B, C)$ is reduced (dually reduced), then $(C, B, A)$, which is equivalent to $(A, B, C)$, is dually reduced (reduced).

THEOR M 6.2. Suppose that $(A, B, C)$ is reduced (dually reduced) form satisfying (6.1). Then at least one of the following inequalities is satisfied,

$$
|A C|<\mathbf{4}|D|, \quad|A(A+B+C)|<\mathbf{4}|D|, \quad|C(A+B+C)|<4|D| .
$$


Proof. Assuming that $(A, B, C)$ is reduced (otherwise we consider $(C, B, A))$, and that

$$
|A C| \geqslant 4|D| \text { and }|A(A+B+C)| \geqslant 4|D|,
$$

we have to derive a contradiction.

Since

$$
B^{2}-D=4 A C,(B+2 A)^{2}-D=4 A(A+B+C),
$$

it follows from $\left({ }^{*}\right)$ that

$$
|B| \geqslant \sqrt{15} \sqrt{|D|} \text { and }|B+2 A| \geqslant \sqrt{15} \sqrt{|D|} .
$$

Also (with a suitable determination of $\sqrt{D}$ )

$$
\xi=(-B+\sqrt{D}) /(2 A) \in \mathcal{J}, \eta=(-B-\sqrt{D}) /(2 A) \in \overline{J^{*}},
$$

and hence at least one of the following cases occur,

(a) $\arg \xi \in[0, \pi]$ and $\arg \eta \in[-\pi / 2,-\pi / 4]$,

(b) $\arg (\xi-1) \in[0, \pi]$ and $\arg (\eta-1) \in[-3 \pi / 4,-\pi / 2]$.

However, for

$$
\frac{\xi}{\eta}-1=\frac{-2 \sqrt{D}}{B+\sqrt{D}}
$$

we get by $(* *)$

$$
\left|\frac{\xi}{\eta}-1\right| \leqslant \frac{2}{\sqrt{15}-1}<\frac{1}{\sqrt{2}}
$$

hence

$$
\left.\arg \frac{\xi}{\eta} \epsilon\right]-\pi / 4, \pi / 4[
$$

contradicting (a).

Similarly, for

$$
\frac{\xi-1}{\eta-1}-1=\frac{-2 \sqrt{D}}{B+2 A+\sqrt{D}}
$$

we get by $\left.{ }^{(* *}\right)$

$$
\left|\frac{\xi-1}{\eta-1}-1\right| \leqslant \frac{2}{\sqrt{15}-1}<\frac{1}{\sqrt{2}}
$$

hence

$$
\arg ((\xi-1) /(\eta-1)) \epsilon]-\pi / 4, \pi / 4[,
$$

contradicting (b).

This proves the theorem. 
CoRolcaRY. For any given $D \in \mathbf{Z}[i]$, where $D$ is not a Gaussian square, there are only finitely many integral forms with discriminant $D$ which are either reduced or dually reduced.

In analogy with a well-known procedure of C. F. Gauss [16] (cf. also L. E. Dickson [8]), we associate to any form $\Phi_{0}$ with roots $\xi_{0}, \eta_{0}$, which is

(a) reduced with $\xi_{0} \in \mathcal{J}$ and $\bar{\eta}_{0} \in \mathcal{J}^{*}$,

or

(b) dually reduced with $\xi_{0} \in \mathcal{J}^{*}$ and $\bar{\eta}_{0} \in \mathcal{J}$,

a double chain of forms

$$
\ldots, \Phi_{-2}, \Phi_{-1}, \Phi_{0}, \Phi_{1}, \Phi_{2}, \ldots
$$

In fact, let

$$
\begin{aligned}
\operatorname{ch} \xi_{0} & =T_{0} T_{1} \ldots ; \operatorname{ch}^{*} \bar{\eta}_{0}=T_{-1} T_{-2} \ldots, \\
\operatorname{ch}^{*} \xi_{0} & =T_{0} T_{1} \ldots ; \operatorname{ch} \bar{\eta}_{0}=T_{-1} T_{-2} \ldots,
\end{aligned}
$$

respectively. [If $\xi_{0}, \eta_{0}$ are not equivalent to real numbers, the chains in $(6.10 \mathrm{a}),(6.10 \mathrm{~b})$ are uniquely determined by Theorems 2.2, 2.2*. Also, if $\Phi_{0}$ is integral, the chains in (6.10a), $(6.10 \mathrm{~b})$ become uniquely determined by requiring these to be purely periodic by Theorem 3.4. In any case we require that $T_{0} \neq V_{1}^{-1}$.] Then we define $\Phi_{n}, n \in Z$, recursively by

$$
\Phi_{n+1}=\Phi_{n} \circ \tilde{T}_{n}, n \in \mathbf{Z}
$$

By (6.11) the roots of $\Phi_{n}$ and $\Phi_{n+1}$ are related as follows,

$$
\xi_{n+1}=t_{n}^{-1}\left(\xi_{n}\right), \eta_{n+1}=t_{n}^{-1}\left(\eta_{n}\right)=\varkappa \circ t_{n} \circ \varkappa\left(\eta_{n}\right),
$$

and hence

$$
\xi_{n}=t_{n}\left(\xi_{n+1}\right), \bar{\eta}_{n+1}=t_{n}\left(\bar{\eta}_{n}\right), n \in \mathbf{Z}
$$

By $(6.10 \mathrm{a}),(6.10 \mathrm{~b}),(6.12)$ it follows that

$$
\xi_{n}=\left[T_{n} T_{n+1} \ldots\right], \bar{\eta}_{n}=\left[T_{n-1} T_{n-2} \ldots\right], n \in \mathbf{Z}
$$

By the very definition of $\left(T_{n}\right)$ it follows, that one of the chains in (6.13) is regular and the other is dually regular. Consequently, each $\Phi_{n}, n \in \mathbf{Z}$, is either reduced or dually reduced.

Notice that in case $\Phi_{0}$ is reduced (dually reduced) and integral, the two-way infinite sequence $\left(T_{n}\right)$ defined by $(6.10 \mathrm{a})$ or $(6.10 \mathrm{~b})$ is periodic by Theorem 3.5 .

The actual calculation of a double chain of forms is facilitated by the following table: 
$\Phi$

$(A, B, C)$

$\Phi \circ \tilde{S}$

$(C,-B-2 C, A+B+C)$

$\Phi \circ \widetilde{S}^{-1} \quad(A+B+C,-2 A-B, A)$

Ф० $\tilde{V}_{1} \quad(A, 2 i A+B,-A+i B+C)$

$\Phi \circ \tilde{V}_{2} \quad(A-i B-C, B-2 i C, C)$

$\Phi \circ \tilde{V}_{3} \quad(-2 i A-(1+i) B-C,(2+2 i) A+3 B+(2-2 i) C,-A+(-1+i) B+2 i C)$

$\Phi \circ \widetilde{E}_{1} \quad(A+(1-i) B-2 i C, i B+(2+2 i) C,-C)$

$\Phi \circ \widetilde{E}_{2} \quad(A,(-2+2 i) A+i B,-2 i A-(1+i) B-C)$

$\Phi \circ \widetilde{E}_{3} \quad(-A, i B, C)$

$\Phi \circ \tilde{C} \quad(A+(1-i) B-2 i C,(-2+2 i) A+3 i B+(2+2 i) C,-2 i A-(1+i) B-C)$

We shall illustrate the computation of double chains of forms in the following examples, which correspond to Examples 2.1, 2.2, 2.3.

Example 6.1. The integral form

$$
\Phi_{0}=(1,-1,1)
$$

is both reduced and dually reduced with

Since

$$
\operatorname{ch} \xi_{0}=\operatorname{ch}^{*} \xi_{0}=\vec{C}
$$

we have

$$
\Phi_{1}=\Phi_{0} \circ \tilde{C}=(-\mathrm{i}, i,-i)=-i \Phi_{0}
$$

$$
\Phi_{n}=(-i)^{n} \Phi_{0}, \quad n \in \mathbf{Z}
$$

Example 6.2. The integral form

$$
\Phi_{0}=(5,-5-2 i, 6+i)
$$

is both reduced and dually reduced with

$$
\operatorname{ch} \xi_{0}=\overline{V_{1} E_{1} C E_{1} V_{1} C}, \quad \operatorname{ch}^{*} \xi_{0}=\overline{C E_{1} C E_{1} C} \vec{C}
$$

Thus precisely two double chains pass through $\Phi_{0}=\Psi_{0}$, namely $\left(\Phi_{n}\right)$, where

$$
\begin{aligned}
& \Phi_{1}=\Phi_{0} \circ \tilde{V}_{1}=(5,-5+8 i, 3-4 i) \\
& \Phi_{2}=\Phi_{1} \circ \tilde{E}_{1}=(7 i, 6-7 i,-3+4 i) \\
& \Phi_{3}=\Phi_{2} \circ \tilde{C}=(7,-7+6 i, 4-3 i)
\end{aligned}
$$




$$
\begin{aligned}
\Phi_{4}= & \Phi_{3} \circ \widetilde{E}_{1}=(5 i, 8-5 i,-4+3 i), \\
\Phi_{5}= & \Phi_{4} \circ \widetilde{V}_{1}=(5 i,-2-5 i, 1+6 i), \\
\Phi_{6}= & \Phi_{5} \circ \widetilde{C}=(5,-5-2 i, 6+i)=\Phi_{0}, \\
& \Phi_{n+6}=\Phi_{n} \text { for all } n \in Z
\end{aligned}
$$

and $\left(\Psi_{n}\right)$, where

$$
\begin{aligned}
\Psi_{1}=\Psi_{0} \circ \widetilde{C}=(-9 i, 6+9 i,-3-4 i), & \\
\Psi_{2}= & \Psi_{1} \circ \widetilde{E}_{1}=(7,-7-8 i, 3+4 i), \\
\Psi_{3}= & \Psi_{2} \circ \widetilde{C}=(-7 i, 8+7 i,-4-3 i), \\
\Psi_{4}= & \Psi_{3} \circ \widetilde{E}_{1}=(9,-9-6 i, 4+3 i), \\
\Psi_{5}= & \Psi_{4} \circ \widetilde{C}=(-5 i, 2+5 i,-1-6 i), \\
\Psi_{6}= & \Psi_{5} \circ \tilde{C}=(-5,5+2 i,-6-i)=-\Psi_{0}, \\
& \Psi_{n+6}=-\Psi_{n} \text { for all } n \in Z .
\end{aligned}
$$

Example 6.3. The integral form

$$
\Phi=(1+2 i, 1,2-i)
$$

is not reduced but dually reduced. However, the form

$$
\Phi_{0}=\Phi \circ \tilde{C}=(-3 i, 3 i, 1-2 i)
$$

is both reduced and dually reduced with

$$
\begin{aligned}
& \operatorname{ch} \xi_{0}=\overline{V_{2} E_{2} V_{2} C E_{1} C V_{3} E_{3} V_{3} C E_{2} C V_{1} E_{1} V_{1} C E_{3} \vec{C}}, \\
& \mathrm{ch}^{*} \xi_{0}=\overline{C E_{2} C V_{1} E_{1} V_{1} C E_{3} C V_{2} E_{2} V_{2} C E_{1} C V_{3} E_{3} \vec{V}_{3}} .
\end{aligned}
$$

Thus precisely two double chains pass through $\Phi_{0}=\Psi_{0}$, namely $\left(\Phi_{n}\right)$, where

$$
\begin{aligned}
\Phi_{1}= & \Phi_{0} \circ \tilde{V}_{2}=(2-i,-4+i, 1-2 i), \\
\Phi_{2}= & \Phi_{1} \circ \widetilde{E}_{2}=(2-i,-3+2 i, 2+i) \\
\Phi_{3}= & \Phi_{2} \circ \tilde{V}_{2}=(2+i,-1-2 i, 2+i), \\
\Phi_{4}= & \Phi_{3} \circ \tilde{C}=(1-4 i, 2+5 i,-1-2 i), \\
\Phi_{5}= & \Phi_{4} \circ \widetilde{E}_{1}=(4+i,-3-4 i, 1+2 i), \\
\Phi_{6}= & \Phi_{5} \circ \widetilde{C}=(-3 i, 3 i, 1-2 i)=\Phi_{0} \circ \tilde{S}^{-1}, \\
& \Phi_{n+6}=\Phi_{n} \circ \tilde{S}^{-1} \text { for all } n \in Z ;
\end{aligned}
$$


and $\left(\Psi_{n}\right)$, where

$$
\begin{aligned}
\Psi_{1}=\Psi_{0} \circ \tilde{C}=(-1-2 i, 3+4 i,-4-i), \\
\Psi_{2}=\Psi_{1} \circ \tilde{E}_{2}=(-1-2 i, 2+5 i, 1-4 i), \\
\Psi_{3}=\Psi_{2} \circ \tilde{C}=(-2-i, 1+2 i,-2-i), \\
\Psi_{4}=\Psi_{3} \circ \tilde{V}_{1}=(-2-i, 3-2 i,-2-i), \\
\Psi_{5}=\Psi_{4} \circ \widetilde{E}_{1}=(1-2 i,-4+i, 2-i), \\
\Psi_{6}=\Psi_{5} \circ \tilde{V}_{1}=(1-2 i, 3 i,-3 i)=\Psi_{0} \circ \tilde{S}^{-1}, \\
\\
\Psi_{n+6}=\Psi_{n} \circ \tilde{S}^{-1} \text { for all } n \in Z .
\end{aligned}
$$

\subsection{Minimum of quadratic forms}

Let $\Phi=(A, B, C)$ be a complex quadratic form. Then

$$
\mu(\Phi)=\inf |\Phi(X, Y)|
$$

the infimum being taken over all $(X, Y) \in \mathbf{Z}[i]^{2} \backslash\{(0,0)\}$, is called the minimum of $\Phi$.

If $\Phi$ does not satisfy $(6.1)$, it is obvious that $\mu(\Phi)=0$.

If $\Phi$ satisfies (6.1), then by Theorem 6.1, Corollary, we may associate (in several ${ }_{4}$ ways) to $\Phi$ a double chain of forms $\left(\Phi_{n}\right)$, each $\Phi_{n}$ being equivalent to $\Phi$, and thus a two-way infinite product

$$
\prod T_{n}=\ldots T_{-2} T_{-1} T_{0} T_{1} T_{2} \ldots,
$$

satisfying the following two conditions,

(i) $T_{n} \in\left\{V_{j}, E_{j}, C\right\}$ for each $n \in \mathbf{Z}$,

(ii) if for $n_{1}<n_{2}$ we have

$$
T_{n_{1}}=E_{j_{1}}, T_{n_{2}}=E_{j_{2}} \quad \text { and } T_{n} \neq E_{j}, \quad(j=1,2,3), \quad \text { for } n_{1}<n<n_{2},
$$

then

$$
\operatorname{card}\left\{n \in \mathbf{Z} \mid n_{1}<n<n_{2}, T_{n}=C\right\} \equiv 1(\bmod 2) .
$$

A two-way infinite product $\Pi T_{n}$ satisfying conditions (i), (ii) is called a regular double chain.

Conversely, for any regular double chain $\Pi T_{n}$ and any $d \in C \backslash\{0\}$, we may define for $n \in \mathbf{Z}$,

$$
\begin{aligned}
\xi_{n} & =\left[T_{n} T_{n+1} \ldots\right], \\
\bar{\eta}_{n} & =\left[T_{n-1} T_{n-2} \ldots\right], \\
\Phi_{n}(X, Y) & =\varepsilon_{n} d\left(\xi_{n}-\eta_{n}\right)^{-1}\left(X-\xi_{n} Y\right)\left(X-\eta_{n} Y\right),
\end{aligned}
$$


where

$$
\varepsilon_{n}=\left\{\begin{array}{l}
\operatorname{det} T_{0} T_{1} \ldots T_{n-1} \quad \text { if } n \geqslant 0 \\
\left(\operatorname{det} T_{-1} T_{-2} \ldots T_{-(-n)}\right)^{-1} \quad \text { if } n<0 .
\end{array}\right.
$$

Then it is easily seen, that $\left(\Phi_{n}\right)$ is a double chain of forms with discriminant $\left(\Phi_{0}\right)=d^{2}$, and $\Pi T_{n}$ is thus a regular double chain associated to $\Phi_{0}$.

For any regular double chain $\prod T_{n}$ we define

$$
K=K\left(\prod T_{n}\right)=\sup _{n \in Z}\left\{\left|\xi_{n}-\eta_{n}\right|,\left|\xi_{n}^{-1}-\eta_{n}^{-1}\right|,\left|\left(\xi_{n}-1\right)^{-1}-\left(\eta_{n}-1\right)^{-1}\right|\right\} .
$$

Also we define a congruence relation in the set of regular double chains by putting

$$
\prod T_{n} \equiv \prod U_{n}
$$

if

$$
U_{n}=\pi\left(T_{\sigma(n)}\right) \text { for all } n \in \mathbf{Z},
$$

where $\sigma: \mathbf{Z} \rightarrow \mathbf{Z}$ is of one of the forms (with a fixed $k \in \mathbf{Z}$ ),

$$
\sigma: n \mapsto n+k \quad \text { or } \sigma: n \mapsto-n+k,
$$

and $\pi:\left\{V_{j}, E_{j}, C\right\} \rightarrow\left\{V_{j}, E_{j}, C\right\}$ is of the form

$$
\pi: V_{j} \mapsto V_{\bar{\pi}(j)}, E_{j} \mapsto E_{\bar{\pi}(j)}, C \mapsto C,
$$

where $\bar{\pi}$ is a permutation of $\{1,2,3\}$.

It is easily verified, that $\equiv$ is an equivalence relation in the set of regular double chains, and that the set

$$
\left\{\left|\xi_{n}-\eta_{n}\right|,\left|\xi_{n}^{-1}-\eta_{n}^{-1}\right|,\left|\left(\xi_{n}-1\right)^{-1}-\left(\eta_{n}-1\right)^{-1}\right| \mid n \in \mathbf{Z}\right\}
$$

appearing in the definition of $K\left(\prod T_{n}\right)$ is invariant under $\equiv$. Consequently

$$
\Pi T_{n} \equiv \prod U_{n} \Rightarrow K\left(\Pi T_{n}\right)=K\left(\prod U_{n}\right) .
$$

Тнеовем 6.3. Suppose that $\Phi$ is a form satisfying (6.1) with discriminant $D$ and minimum $\mu$, and that $\prod T_{n}$ is a regular double chain associated to $\Phi$. Then

A. $\sqrt{|D|} / \mu \geqslant K\left(\Pi T_{n}\right)$.

B. If, in addition, $\Phi$ is proportional to an integral form, then

$$
\sqrt{|D|} / \mu=K\left(\Pi T_{n}\right) .
$$

Proof. The double chain $\left(\Phi_{n}\right)$ defined above by

$$
\Phi_{n}(X, Y)=\varepsilon_{n} d\left(\xi_{n}-\gamma_{n}\right)^{-1}\left(X-\xi_{n} Y\right)\left(X-r_{i n} Y\right)
$$


where $\left|\varepsilon_{n}\right|=1$ and $d^{2}=D$, has each $\Phi_{n}$ equivalent to $\Phi$. Consequently for each $n \in Z$, we have

$$
\begin{aligned}
\mu=\mu\left(\Phi_{n}\right) \leqslant \min \left(\left|\Phi_{n}(1,0)\right|,\left|\Phi_{n}(0,1)\right|,\left|\Phi_{n}(1,1)\right|\right) & \\
= & \sqrt{|D|} \min \left(\left|\xi_{n}-\eta_{n}\right|^{-1},\left|\xi_{n}^{-1}-\eta_{n}^{-1}\right|^{-1},\left|\left(1-\xi_{n}\right)^{-1}-\left(1-\eta_{n}\right)^{-1}\right|^{-1}\right) .
\end{aligned}
$$

This proves part A.

The proof of part B is essentially identical to that of Theorem 2.7.

COROLLARY. If $\left(\Phi_{n}\right)$ with $\Phi_{n}=\left(A_{n}, B_{n}, C_{n}\right)$ is a periodic double chain of forms satis. fying (6.1), then

$$
\mu\left(\Phi_{0}\right)=\min \left(\left|A_{n}\right|,\left|C_{n}\right|,\left|A_{n}+B_{n}+C_{n}\right|\right),
$$

where it suffices to extend the minimum over a period.

Example 6.4. Using formula (6.15) for the forms $\Phi_{0}$ occurring in Examples 6.1, 6.2, 6.3, we obtain.

$$
\begin{array}{ll}
\mu((1,-1,1))=1, & \sqrt{|D|} / \mu=\sqrt{3}=1.7320 \ldots \\
\mu((5,-5+2 i, 6+i))=5, & \sqrt{|D|} / \mu=\sqrt{4-5^{-2}}=1.9899 \ldots \\
\mu((1+2 i, 1,2-i))=\sqrt{5}, & \sqrt{|D|} / \mu=\sqrt{\frac{3}{5} \sqrt{41}}=1.9599 \ldots
\end{array}
$$

A main problem about the minimum of complex binary quadratic forms is that of determining all forms $\Phi$ with $\sqrt{|D|} / \mu<2$.

We shall solve this problem in two steps, using Theorem 6.3:

$1^{\circ}$. In section 6.3 we describe rather precisely those regular double chains $\Pi T_{n}$, which have $K\left(\Pi T_{n}\right)<2$.

$2^{\circ}$. In section 6.4 we solve the remaining problems by means of Theorem 5.3.

\subsection{Regular double ehains $\prod T_{n}$ with $K<2$}

Throughout this section we deduce restrictions on a regular double chain $\Pi T_{n}$ in order to satisfy the condition $K<2$, by the following two arguments:

(1) If $T_{n} T_{n+1} \ldots$ is a regular chain and $T_{n-1} T_{n-2} \ldots$ is a dually regular chain, then by Theorems 2.1, 2.1*,

$$
\begin{aligned}
& \xi_{n}=\left[T_{n} T_{n+1} \ldots\right] \in F\left(T_{n} T_{n+1} \ldots T_{n+h-1}\right), \\
& \bar{\eta}_{n}=\left[T_{n-1} T_{n-2} \ldots\right] \in F^{*}\left(T_{n-1} \ldots T_{n-k}\right),
\end{aligned}
$$

5-752903 Acta mathematica 134. Imprimé le 31 Juillet 1975 
for all $h, k \in \mathbf{N}_{0}$. [Notice, that we write $F\left(T_{n} \ldots T_{n+h-1}\right)$ instead of $F\left(t_{n} \circ \ldots \circ t_{n+h-1}\right)$, etc.]. However, since

$$
\left|\xi_{n}-\eta_{n}\right| \leqslant \mathrm{K}<2
$$

we must have

$$
d\left(F\left(T_{n} T_{n+1} \ldots T_{n+h-1}\right), \overline{F^{*}}\left(T_{n-1} \ldots T_{n-k}\right)\right)<2
$$

for all $h, k \in \mathbf{N}_{0}$; here $d$ denotes the euclidean distance between the two sets involved.

Similarly, if $T_{n} T_{n+1} \ldots$ is a dually regular chain and $T_{n-1} T_{n-2} \ldots$ is a regular chain, then we must have

$$
d\left(F\left(T_{n-1} \ldots T_{n-k}\right), \overline{F^{*}}\left(T_{n} T_{n+1} \ldots T_{n+n-1}\right)\right)<2
$$

for all $h, k \in \mathbf{N}_{\mathbf{0}}$.

(2) If, by argument (1), we have excluded a product $U_{n-k} \ldots U_{n-1} U_{n} U_{n+1} \ldots U_{n+h-1}$ from appearing as a subproduct in $\Pi T_{n}$, then by (6.14) any product congruent to $U_{n-k} \ldots U_{n-1} U_{n} U_{n+1} \ldots U_{n+h-1}$, i.e. obtained by applying a permutation $\pi:\left\{V_{j}, E_{j}, C\right\} \rightarrow$ $\left\{V_{j}, E_{j}, C\right\}$ induced by a permutation $\bar{\pi}$ of $\{1,2,3\}$, and possibly a reversion, is also excluded as a subproduct of $\Pi T_{n}$.

We shall often refer to an application of argument (2) by the phrase: "using congruence ...".

The first four lemmas will deal with $V$-subproducts i.e. subproducts of $\Pi T_{n}$ consisting only of $V_{j}$ 's. By the very definition of a regular double chain, a maximal $V$-subproduct, if finite, will be of the form

$$
U_{1} V_{j_{3}}^{\prime \prime} V_{j_{2}} \ldots V_{j_{m}} U_{2}
$$

with $U_{1}, U_{2} \in\left\{E_{j}, C\right\}$, and either $U_{1}=C$ or $U_{2}=C$.

LEMMA 6.1. The regular double chain $\Pi T_{n}$ contains, modulo congruence, only the following $V$-subproducts,

$$
V_{1}, V_{1} V_{2}, V_{1} V_{2} V_{3}
$$

Proof. Using congruence, we get the result concerning $V$-subproducts of length 2 from

$$
d\left(F\left(V_{1} V_{1}\right), \overline{F^{*}}(I)\right)=2 .
$$

Using this result and congruence, we obtain the result concerning $V$-subproducts of length 3 by excluding $V_{1} V_{2} V_{1}$ as a subproduct. Hence assume that $V_{1} V_{2} V_{1}$ is a subproduct of $\Pi T_{n}$, and assume, using congruence, that the maximal $V$-subproduct containing $V_{1} V_{2} V_{1}$ is either of the form (6.16) with $U_{1}=C$ or is infinite to the left. Since

$$
d\left(F\left(V_{1} V_{2} V_{1}\right), \overline{F^{*}}\left(V_{3} T\right)\right)>2 \text { for } T=V_{1}, V_{2}, C
$$


and $V_{3} V_{3}$ has already been ex sluded, it follows that $\Pi T_{n}$ either contains a subproduct congruent to $C V_{1} V_{2} V_{1}$ or a subproduct congruent to $\overleftarrow{V_{1} V_{2}}$, the arrow indicating periodicity to the left. However, since

$$
\begin{aligned}
& d\left(F\left(V_{1} V_{2} V_{1}, \overline{F^{*}}(C)\right)>2,\right. \\
& d\left(\left[\overrightarrow{V_{1} \vec{V}_{2}}\right],\left[\overline{\overrightarrow{V_{2}} \overrightarrow{V_{1}}}\right]\right)=\sqrt{5}>2,
\end{aligned}
$$

both possibilities are exriuded. This proves the result concerning $V$-subproducts of length 3 .

Finally, if $\Pi T_{n}$ contains a $V$-subproduct of length $\geqslant 4$, then by the same argument as above, either $\Pi T_{n}$ contains a subproduct congruent to $C V_{1} V_{2} V_{3} V_{11}$ or a subproduct congruent to $\overline{V_{1}} \overline{V_{2} V_{3}}$. However, since

$$
\begin{gathered}
d\left(F\left(V_{1} V_{2} V_{3} V_{1}\right), \overline{F^{*}}(C)\right)>2, \\
d\left(\left[\overline{V_{1} V_{2} \vec{V}_{3}}\right],\left[\overline{V_{3} \vec{V}_{2} \vec{V}_{1}}\right]\right)=\sqrt[4]{20}>2,
\end{gathered}
$$

both alternatives are impossible. This excludes the existence of a $V$-subproduct of length $\geqslant 4$

L Е м А 6.2. The regular double chain $\Pi T_{n}$ contains no $V$-subproduct of length 3.

Proof. Using congruence, we need only by Lemma 6.1 exclude $C V_{1} V_{2} V_{3} U_{1} U_{2}$ with $U_{1} \in\left\{E_{3}, C\right\}$, as a subproduct of $\Pi T_{n}$. However, this follows from

$$
\begin{gathered}
d\left(F\left(V_{1} C\right) \backslash F\left(V_{1} C V_{1}^{2}\right), \overline{F^{*}}\left(V_{3} V_{2} C\right)\right)>2, \\
d\left(F\left(V_{1} V_{2} E_{2}\right), \overline{F^{*}}\left(V_{3} C\right)\right)>2, \\
d\left(F\left(V_{1} V_{2} V_{3} E_{2}\right), \overline{F^{*}}(C)\right)>2, \\
d\left(F\left(V_{1} E_{2} V_{1}\right) \backslash F\left(V_{1} E_{2} V_{1} V_{3}^{2}\right), \overline{F^{*}}\left(V_{3} V_{2} C\right)\right)>2, \\
d\left(F\left(V_{1} E_{2} V_{2}\right), \overline{F^{*}}\left(V_{3} V_{2} C\right)\right)>2, \\
d\left(F\left(V_{1} V_{2} E_{3} V_{1}\right), \overline{F^{*}}\left(V_{3} C\right)\right)>2, \\
d\left(F\left(V_{1} V_{2} E_{3} C\right), \overline{F^{*}}\left(V_{3} C\right)\right)>2,
\end{gathered}
$$

which excludes $U_{1}=C, \quad U_{1}=E_{3}, \quad U_{1}=E_{2}, \quad U_{1} U_{2}=E_{1} V_{3}, \quad U_{1} U_{2}=E_{1} V_{1}, U_{1} U_{2}=E_{1} V_{2}$, $U_{1} U_{2}=E_{1} C$, respectively.

LEMмA 6.3. The regular double chain $\Pi T_{n}$ contains no V-subproduct of length 2. 
Proof. Using congruence, we need only by Lemma 6.2 exclude $C V_{1} V_{2} U_{1} U_{2} U_{3} \ldots$ with $U_{1} \in\left\{E_{j}, C\right\}$, as a subproduct of $\Pi T_{n^{*}}$. Since

$$
\begin{aligned}
& d\left(F\left(V_{1} V_{2} E_{2}\right), \overline{F^{*}}(C)\right)=2, \\
& d\left(F\left(V_{1} C\right) \backslash F\left(V_{1} C V_{1}^{2}\right), \overline{F^{*}}\left(V_{3} C\right)\right)>2, \\
& d\left(F\left(V_{1} V_{2} E_{3} V_{1}\right), \overrightarrow{F^{*}}(C)\right)>2, \\
& d\left(F\left(V_{1} E_{2} V_{1}\right) \backslash F\left(V_{1} E_{2} V_{1} V_{3}^{2}\right), \overline{F^{*}}\left(V_{3} C\right)\right)>2, \\
& d\left(F\left(V_{1} E_{2} V_{2}\right) \backslash F\left(V_{1} E_{2} V_{2} V_{3}^{2}\right), \overline{F^{*}}\left(V_{3} C\right)\right)>2, \\
& d\left(F\left(V_{1} V_{2} E_{3} C U\right), \overline{F^{*}}(C)\right)>2 \text { for } U \in\left\{V_{2}, V_{3}, E_{1}, C\right\} \text {, } \\
& d\left(F\left(V_{1} E_{2} C V_{3}\right), \overline{F^{*}}\left(V_{3} C\right)\right)>2, \\
& d\left(F\left(V_{1} E_{2} C E_{1}\right) \backslash F\left(V_{1} E_{2} C E_{1} V_{2}^{2}\right), \overline{F^{*}}\left(V_{3} C\right)\right)>2, \\
& d\left(F\left(V_{1} E_{2} C E_{2}\right) \backslash F\left(V_{1} E_{2} C E_{2} V_{1}^{2}\right), \overline{F^{*}}\left(V_{3} C\right)\right)>2,
\end{aligned}
$$

we can exclude $U_{1}=E_{2}, U_{1}=C, U_{1} U_{2}=E_{3} V_{1}, U_{1} U_{2}=E_{3} V_{2}, U_{1} U_{2}=E_{3} V_{3}, U_{1} U_{2} U_{3}=$ $E_{3} C U$ with $U \in\left\{V_{2}, V_{3}, E_{1}, C\right\}, U_{1} U_{2} U_{3}=E_{3} C V_{1}, U_{1} U_{2} U_{3}=E_{3} C E_{2}, U_{1} U_{2} U_{3}=E_{3} C E_{3}$, respectively. Consequently $U_{1}=E_{1}$ is the only possibility.

Since

$$
d\left(F\left(V_{1} E_{3} U\right), \overline{F^{*}}\left(V_{3} C\right)\right) \text { for } U \in\left\{V_{3}, V_{1}, C\right\},
$$

we can exclude $U_{2} \in\left\{V_{1}, V_{2}, C\right)$. Consequently $U_{1} U_{2}=E_{1} V_{3}$ is the only possibility.

Now $U_{3}=V_{3}$ contradicts Lemma 6.1. Further $U_{3}=V_{2}$ implies $U_{4}=C$ by Lemma 6.2 and the definition of a regular double chain, hence $U_{1} U_{2} U_{3} U_{4}=E_{1} V_{3} V_{2} C \equiv C V_{1} V_{2} E_{3}$, which was exeluded above. Similarly, $U_{3}=V_{1}$ implies $U_{4}=C$, however, this is also impos. sible, since

$$
d\left(F\left(V_{1} E_{3} V_{2} V_{3}\right) \backslash F\left(V_{1} E_{3} V_{2} V_{3} V_{2}^{2}\right), \overline{F^{*}}\left(V_{3} C\right)\right)>2 .
$$

Consequently $U_{1} U_{2} U_{3}=E_{1} V_{3} C$ is the only possibility.

Since

$$
\begin{gathered}
d\left(F\left(V_{1} E_{2} V_{3} V_{2} C\right), \overline{F^{*}}(C U)\right)>2 \text { for } U \in\left\{V_{2}, V_{3}, E_{1}, E_{2}, C\right\}, \\
d\left(F\left(V_{1} E_{3} V_{2} C V_{2}\right), \overline{F^{*}}\left(V_{3} C\right)\right)>2,
\end{gathered}
$$

we can exclude $U_{4} \in\left\{V_{1}, V_{2}, E_{3}, E_{1}, C\right\}, U_{4}=V_{3}$, respectively. Consequently $U_{1} U_{2} U_{3} U_{4}=$ $E_{1} V_{3} C E_{2}$ is the only possibility.

Since

$$
d\left(F\left(V_{1} E_{3} V_{2} C E_{1} U\right), \overline{F^{*}}\left(V_{3} C\right)\right)>2 \text { for } U \in\left\{V_{1}, V_{2}, C\right\}
$$

we can exclude $U_{5} \in\left\{V_{2}, V_{3}, C\right\}$. Consequently $U_{1} U_{2} U_{3} U_{4} U_{5}=E_{1} V_{3} C E_{2} V_{1}$ is the only possibility. 
If $U_{6}=V_{j}$, then $C E_{2} V_{1} V_{j} C$ would be a subproduct of $\Pi T_{n}$, however, this possibility has already been excluded in course of the proof. Consequently $U_{1} U_{2} U_{3} U_{4} U_{5} U_{6}=$ $E_{1} V_{3} C E_{2} V_{1} C$ is the only possibility. However, this is also excluded since

$$
d\left(F\left(V_{1} E_{2} C V_{3}\right), \overline{F^{*}}(C)\right)>2 .
$$

This proves Lemma 6.3.

LEMma 6.4. The regular double chain $\Pi T_{n}$ contains $V_{j}$ only in the following combinations,

$$
C V_{j} E_{j} C, \quad C E_{j} V_{j} C, \quad C V_{j} E_{j} V_{j} C
$$

Proof. Using congruence, we need only by Lemma 6.3 consider subproducts of $\Pi T_{n}$ of the form ... $W_{3} W_{2} W_{1} C V_{1} U_{1} U_{2} U_{3} \ldots$, where $U_{1} \in\left\{E_{j}, C\right\}$. Since

$$
d\left(F\left(V_{1} C\right), \overline{F^{*}}(C)\right)=2 \text {, }
$$

we may assume, using congruence, that either (a) $U_{1}=E_{2}$ or (b) $U_{1}=E_{1}$.

Case (a), $U_{1}=E_{2}$. Since

$$
\begin{aligned}
& d\left(F\left(V_{1} E_{2} V_{1}\right), \overline{F^{*}}(C)\right)=2, \\
& d\left(F\left(V_{1} E_{2} V_{2}\right), \overline{F^{*}}(C)\right)>2,
\end{aligned}
$$

either (aa) $U_{2}=C$ or (ab) $U_{2}=V_{3}$.

Subcase (aa), $\mathrm{U}_{1} \mathrm{U}_{2}=E_{2} C$. Since

$$
\begin{gathered}
d\left(F^{\prime}\left(V_{1} E_{2} C U\right), \overline{F^{*}}(C)\right) \geqslant 2 \text { for } U \in\left\{V_{1}, V_{3}, E_{1}, E_{2}, C\right\}, \\
d\left(F\left(V_{1}\right), \overline{F^{*}}\left(C E_{1}\right)\right)=2,
\end{gathered}
$$

we must have $U_{3}=E_{3}$.

Suppose that $U_{4}=V_{j}$, then $U_{5}=C$ by Lemma 6.3 and the definition of a regular double chain. However, $U_{1} U_{2} U_{3} U_{4} U_{5}=E_{2} C E_{3} V_{2} C \equiv C V_{1} E_{2} C E_{1}$ was excluded above, hence $U_{4} \neq V_{2}$, and since

$$
d\left(F\left(V_{1} E_{2} C E_{3} V_{j} C\right), \overline{F^{*}}(C)\right)>2 \text { for } j \in\{1,3\}
$$

also $U_{4} \notin\left\{V_{1}, V_{3}\right\}$. Consequently $U_{4}=C$.

Since

$$
d\left(F\left(E_{3} C E_{1} C\right), \overline{F^{*}}\left(V_{2}\right)\right)>2,
$$

this leads to a contradiction; thus subcase (aa) is excluded. We use this fact tacitly in the sequel. 
Subcase (ab), $U_{1} U_{2}=E_{2} V_{3}$. Then $U_{3^{\circ}}=C$ by Lemma 6.3 . Since

$$
\begin{gathered}
d\left(F\left(V_{1} E_{2} V_{3} C\right), F^{* *}\left(C W_{1}\right)\right)>2 \text { for } W_{1} \in\left\{V_{3}, E_{1}, C\right\}, \\
d\left(F\left(V_{1}\right), \overline{F^{*}}\left(C V_{3} E_{1}\right)\right)=2,
\end{gathered}
$$

we can exclude $W_{1} \in\left\{V_{3}, E_{1}, C\right\}, W_{1}=V_{2}$, respectively. Consequently either (aba) $W_{1}=E_{2}$ or (abb) $W_{1}=V_{1}$ or (abc) $W_{1}=E_{3}$.

Subcase (aba), $W_{1} C V_{1} U_{1} U_{2} U_{3}=E_{2} C V_{1} E_{2} V_{3} C$. Then either $W_{2}=C$ or $W_{3} W_{2}=C V_{2}$ by Lemma 6.3 and the exclusion of subcase (aa). By symmetry $U_{4} \in\left\{E_{2}, V_{3}, E_{1}\right\}$, and in case $U_{4}=E_{2}$, either $U_{5}=C$ or $U_{5} U_{6}=V_{2} C$.

Since

$$
d\left(F\left(V_{1} E_{2} V_{3} C\right), \overline{F^{*}}\left(C E_{2} V_{2} C\right)\right)>2,
$$

we can exclude $W_{3} W_{2} W_{1}=C V_{2} E_{2}$, and hence by symmetry also $U_{4} U_{5} U_{6}=E_{2} V_{2} C$. Since

$$
d\left(F\left(V_{1} E_{2} V_{3} C U_{4} U_{5} U_{6}\right), \overline{F^{*}}\left(C E_{2} C\right)\right)>2
$$

for $U_{4}=V_{3}, U_{4} U_{5}=E_{1} C, U_{4} U_{5} U_{6}=E_{1} V_{1} C, U_{4} U_{5}=E_{2} C$, we can exclude $W_{2} W_{1}=C E_{2}$, and hence by symmetry also $U_{4} U_{5}=E_{2} C$. Thus subcase (aba) is excluded, and necessarily $U_{4} \in\left\{V_{3}, E_{1}\right\}$.

Subcase (abb), $W_{1} C V_{1} U_{1} U_{2} U_{3}=V_{1} C V_{1} E_{2} V_{3} C$. Since

$$
\begin{gathered}
d\left(F\left(V_{1} E_{2} V_{3} C\right), \overline{F^{*}}\left(C V_{1} E_{1}\right)\right)>2, \\
d\left(F\left(V_{1} E_{2} V_{3} C U_{4} U_{5} U_{6}\right), \overline{F^{*}}\left(C V_{1} E_{2} V_{3} C\right)\right)>2
\end{gathered}
$$

for $U_{4}=V_{3}, U_{4} U_{5}=E_{1} C, U_{4} U_{5} U_{6}=E_{1} V_{1} C$, and

$$
d\left(F\left(V_{1} C\right), \overline{F^{*}}(C)\right)=\mathbf{2}
$$

the only possibility remaining is $W_{2}=E_{3}$, and consequently $W_{4} W_{3}=C V_{2}$.

Since

$$
K\left(\overleftarrow{C} \overline{V_{3} E_{1} V_{2} C V_{2}} \bar{b}_{3} V_{1} C V_{1} E_{2} \vec{V}_{3}\right)=\sqrt{\frac{21}{5}}>2
$$

we may assume, using congruence and $U_{4} \in\left\{V_{3}, E_{1}\right\}$, that $U_{4}=E_{1}$ and consequently $U_{5}=C$ or $U_{5} U_{6}=V_{1} C$. However, this leads to a contradiction, since

$$
d\left(F\left(V_{3} E_{2} V_{1} C V_{1} E_{3} V_{2} C\right), \overline{F^{*}}\left(C E_{1} U_{5} U_{6}\right)\right)>2
$$

for $U_{5}=C$ and $U_{5} U_{6}=V_{1} C$. Thus subcase (abb) is excluded, and by symmetry also $U_{4}=V_{3}$ is excluded. 
Subcase (abc), $W_{1} C V_{1} U_{1} U_{2} U_{3} U_{4}=E_{3} C V_{1} E_{2} V_{3} C E_{1}$. Then $W_{2}=C$ or $W_{3} W_{2}=C V_{3}$ and $U_{5}=C$ or $U_{5} U_{6}=V_{1} C$. However, all four combinations are impossible, since

$$
d\left(F\left(V_{3} E_{2} V_{1} C E_{3} W_{2} W_{3}\right), \overline{F^{*}}\left(C E_{1} U_{5} U_{6}\right)\right)>2
$$

in all four cases. This excludes subcase (abc).

Consequently subcase (ab) is excluded, and thus case (a) is excluded.

Case (b), $U_{1}=E_{1}$. Then by Lemma 6.3 and the exclusion of subcase (aa), we conclude that either $U_{2}=C$ or $U_{2} U_{3}=V_{1} C$. This completes the proof of Lemma 6.4.

Lемма 6.5. The regular double chain $\Pi T_{n}$ contains $E_{j}$ only in the following combinations,

$$
C E_{j} C, C V_{j} E_{j} C, C E_{j} V_{j} C, C V, E_{j} V_{j} C .
$$

Proof. The result is an immediate consequence of Lemma 6.4 and the definition of a regular double chain.

L Е м м 6.6. The regular double chain $\Pi T_{n}$ contains $C V, E_{j} V_{j} C$ as a subproduct only if either

$$
\prod T_{n}=\overleftarrow{C V_{1} E_{1} V_{1} C E_{2} C V_{3} E_{3} V_{3} C E_{1} C V_{2} E_{2} V_{2} C E_{3}}
$$

or

$$
\prod T_{n}=\overleftarrow{C} \overline{V_{1} E_{1} V_{1} C E_{3}} C V_{2} E_{2} V_{2} C E_{1} C V_{3} E_{3} V_{3} C E_{2} .
$$

Conversely, for each of these regular double chains

$$
K=K\left(\prod T_{n}\right)=\sqrt{\frac{3}{5} \sqrt{41}}=1.9599 \ldots .
$$

Proof. Using congruence, we may assume that $\Pi T_{n}$ contains the subproduct $\ldots W_{3} W_{2} W_{1} C V_{1} E_{1} V_{1} C U_{1} U_{2} U_{3} \ldots$. Since

$$
\begin{gathered}
d\left(F^{\prime}\left(V_{1} E_{1} V_{1} C\right), \overline{F^{*}}(C X)\right)>2 \text { for } X \in\left\{V_{2}, V_{3}, E_{1}, C\right\}, \\
d\left(F\left(V_{1} E_{1} V_{1} C\right), \overline{F^{*}}\left(C V_{1} E_{1}\right)\right)=2,
\end{gathered}
$$

we conclude that $U_{1}, W_{1} \in\left\{E_{2}, E_{3}\right\}$. Since also

$$
d\left(F\left(E_{3} V_{3} C E_{1} C\right) \cup F\left(E_{3} V_{3} C E_{1} V_{1} C\right), \overline{F^{*}}\left(V_{3} C E_{1} C\right) \cup \overline{F^{*}}\left(V_{3} C E_{1} V_{1} C\right)\right)>2,
$$

we conclude by Lemma 6.5 that $U_{1} \neq W_{1}$. Hence, using congruence, we may assume that $U_{1}=E_{2}, W_{1}=E_{3}$.

Using Lemma 6.5, we obtain from

$$
d\left(F\left(V_{1} E_{1} V_{1} C E_{2} C\right) \cup F\left(V_{1} E_{1} V_{1} C E_{2} V_{2} C\right), \overline{F^{*}}\left(C E_{3} V_{3} C\right)\right)>2,
$$


that $W_{3} \neq V_{3}$; by symmetry, also $U_{2} \neq V_{2}$. Hence by Lemma 6.5 , it follows that $U_{2}=W_{2}=C$.

Since

$$
\begin{gathered}
d\left(F\left(V_{1}\right), \overline{F^{*}}\left(C E_{1}\right)\right)=2, \\
d\left(F\left(C^{2}\right), \overline{F^{*}}\left(C E_{1} C V_{3} E_{3} V_{3} C\right)\right)>2, \\
d\left(F\left(E_{3} C V_{2} E_{2}\right), \overline{F^{*}}\left(C V_{2} E_{2} V_{2} C\right)\right)>2, \\
d\left(F\left(E_{3} C E_{1} C\right) \cup F\left(E_{3} C E_{1} V_{1} C\right), \overline{F^{*}}\left(C V_{2} E_{2} V_{2} C\right)\right)>2, \\
d\left(F\left(E_{2} C V_{1} E_{1} V_{1} C\right), \overline{F^{*}}\left(C E_{1} C\right) \cup \overline{F^{*}}\left(C E_{1} V_{1} C\right)\right)>2, \\
d\left(F\left(E_{3} C V_{2} E_{2} V_{2} C\right), \overline{F^{*}}\left(C E_{3} C\right) \cup \overline{F^{*}}\left(C E_{3} V_{3} C\right)\right)>2,
\end{gathered}
$$

we can exclude $U_{3}=V_{2}, U_{3}=C, U_{3}=V_{1}, U_{3}=E_{3}, U_{3}=E_{1}, U_{3}=E_{2}$, respectively, by Lemmas 6.4, 6.5. Consequently $U_{3}=V_{3}$, and hence by Lemma 6.4, either $U_{4} U_{5}=E_{3} C$ or $U_{4} U_{5} U_{6}=$ $E_{3} V_{3} C$. However, since

$$
d\left(F\left(V_{2} E_{2} C\right), \overline{F^{*}}\left(C E_{1} C V_{3} E_{3} V_{3} C\right)\right)>2,
$$

we can exclude the first of these possibilities, and hence $U_{4} U_{5} U_{6}=E_{3} V_{3} C$.

Repeating the argument used above in both directions, we conclude that necessarily

$$
\Pi T_{n} \equiv \widetilde{C V_{1} E_{1} V_{1} C E_{2} C V_{3} E_{3} V_{3} C E_{1} C V_{2} E_{2} V_{2} C E_{3}}
$$

By (6.14), Theorem 6.3 B and Examples 6.3, 6.4, this yields the result.

LеммА 6.7. The regular double chain $\Pi T_{n}$ contains $\overleftarrow{C}$ or $\vec{C}$ as a subproduct only if $\Pi T_{n}=\overleftrightarrow{C}$. Conversely

$$
K=K(\overleftrightarrow{C})=\sqrt{3}=1.7320 \ldots
$$

Proof. That $K(\vec{C})=\sqrt{3}$ follows from Theorem $6.3 \mathrm{~B}$ and Examples 6.1, 6.4. Hence assuming by congruence, that $\Pi T_{n}=\overleftarrow{C} U_{1} U_{2} U_{3} \ldots$ with $U_{1} \in\left\{V_{1}, E_{1}\right\}$, we have to reach a contradiction.

By Lemmas 6.4, 6.5, 6.6, either (a) $U_{1} U_{2}=E_{1} C$ or (b) $U_{1} U_{2} U_{2}=E_{1} V_{1} C$ or (c) $U_{1} U_{2} U_{3}$ $=V_{1} E_{1} C$.

Case (a). This possibility is excluded at once by

$$
d\left(F\left(C^{4}\right), \overline{F^{*}}\left(C E_{1} C\right)\right)>2 .
$$

Case (b). Since $K\left(\overleftarrow{C} E_{1} V_{1} \vec{C}\right)=2$, we may assume, that

$$
\overleftarrow{C U_{1}} U_{2} U_{3} \ldots=\overleftarrow{C E_{1}} V_{1} C^{k} X_{1} X_{2} X_{3} \ldots
$$

where $X_{1} \neq C$. By the definition of a regular double chain together with Lemmas 6.4, 6.5, it follows that $k=2 m-1, m \in \mathbf{N}$. 
However,

$$
\left.d\left([\vec{C}], \overline{F^{*}}\left(C E_{1} V_{1} C^{2 m-1} X_{1} X_{2} X_{3}\right)\right)>d(\overrightarrow{[C}], \overline{\left[C E_{1} V_{1} \vec{C}\right]}\right)=2
$$

for $X_{1}=V_{1}, X_{1} X_{2}=E_{2} C, X_{1} X_{2} X_{3}=E_{2} V_{2} C, X_{1} X_{2}=E_{3} C, X_{1} X_{2} X_{3}=E_{3} V_{3} C$, and hence by Lemmas 6.4, 6.5, 6.6, we have $X_{1}{ }^{\dagger} \notin\left\{V_{1}, E_{2}, E_{3}\right\}$.

Similarly

$$
\left.d\left(\left[V_{1} E_{1} \vec{C}\right], \overline{F^{*}}\left(C^{2 m-1} X_{1} X_{2} X_{3}\right)\right)>d\left(\left[V_{1} E_{1} \vec{C}\right], \overline{\overline{[C}}\right]\right)=2
$$

for $X_{1} X_{2} X_{3}=V_{2} E_{2} C, X_{1} X_{2} X_{3}=V_{3} E_{3} C, X_{1}=E_{1}$, and hence by Lemmas 6.4, 6.5, 6.6, we have $X_{1} \notin\left\{V_{2}, V_{3}, E_{1}\right\}$.

This excludes case (b).

Case (c). This possibility is excluded similarly to case (b).

From Lemmas 6.4, 6.5, 6.6, 6.7 and the definition of a regular double chain we obtain the following structure theorem.

THEOR M 6.4. Suppose that the regular double chain $\Pi T_{n}$ with $K\left(\Pi T_{n}\right)<2$ is different from $\overleftrightarrow{C}$ and from

$$
\overparen{C V_{1} E_{1} V_{1} C E_{2} C V_{3} E_{3} V_{3} C E_{1} C V_{2} E_{2} V_{2} C E_{3}}
$$

and

$$
\overleftarrow{C} \overline{V_{1} E_{1} V_{1} C E_{3} C V_{2} E_{2} V_{2} C E_{1} C V_{3} E_{3} V_{3} C E_{2}}
$$

Then $\prod T_{n}$ is of the form

$$
\ldots C^{2 m_{-1}-1} T_{1}^{(-1)} \ldots T_{k_{-1}}^{(-1)} C^{2 m_{0}-1} T_{1}^{(0)} \ldots T_{k_{0}}^{(0)} C^{2 m_{1}-1} \ldots
$$

where $m_{r} \in \mathbf{N}$ for all $r \in \mathbf{Z}$ and $T_{1}^{(r)} \ldots T_{k_{r}}^{(r)}$ for each $r \in \mathbf{Z}$ is one of the nine products,

$$
E_{j}, V_{j} E_{j}, E_{j} V_{j} \text {, with } j \in\{1,2,3\} \text {. }
$$

LEM А 6.8. The regular double chain $\Pi T_{n}$ contains

$$
C^{2 l-1} E_{j} C^{2 m-1}
$$

as a subproduct only if $(l, m) \in\{(1,1),(2,1),(1,2)\}$.

Proof. Using congruence, we may assume that $l \geqslant m$ and $j=1$. Then $l \geqslant 3$ is excluded by

$$
d\left(F\left(C^{2 t-2}\right), \overline{F^{*}}\left(C E_{1} C\right)\right) \geqslant d\left(F\left(C^{4}\right), \overline{F^{*}}\left(C E_{1} C\right)\right)>2,
$$

and $(l, m)=(2,2)$ is excluded by

$$
d\left(F\left(C^{2}\right), \overline{F^{*}}\left(C E_{1} C^{3}\right)\right)>2 .
$$


Lемм А 6.9. The regular double chain $\Pi T_{n}$ contains $C^{3} E_{j} C$ or $C E_{j} C^{3}$ as a subproduct only in the following combinations,

$$
E_{j} C^{3} E_{j} C E_{j} \text { or } E_{j} C E_{j} C^{3} E_{j}
$$

Proof. Using congruence, we get the result from Theorem 6.4 and Lemma 6.8, since

$$
\begin{aligned}
& d\left(F\left(C^{2}\right), \overline{F^{*}}\left(C E_{1} C X_{1} X_{2} X_{3}\right)\right)>2, \\
& d\left(F\left(C^{2} X_{1} X_{2} X_{3}\right), \overline{F^{*}}\left(C E_{1} C\right)\right)>2,
\end{aligned}
$$

are both satisfied for $X_{1}=V_{1}, X_{1} X_{2}=E_{k} C(k=2,3), X_{1} X_{2} X_{3}=E_{k} V_{k} C(k=2,3), X_{1} X_{2} X_{3}$ $=V_{k} E_{k} C(k=2,3)$.

LE M M 6.10. The regular double chain $\Pi T_{n}$ does not contain any of the following products as a subproduct,

$$
C E_{j} C E_{k} V_{k} C, \quad C V_{k} E_{k} C E_{j} C \quad(j \neq k) .
$$

Proof. Using congruence, we obtain the result from

$$
d\left(F\left(E_{2} V_{2} C\right), \overline{F^{*}}\left(C E_{1} C\right)\right)>2 .
$$

L Емм А 6.11. The regular double chain $\Pi T_{n}$ does not contain any of the following products as a subproduct,

$$
C E_{j} C V_{k} E_{k} C, \quad C E_{k} V_{k} C E_{j} C \quad(j \neq k)
$$

Proof. Using congruence, we need only exclude $C E_{1} C V_{2} E_{1} C X_{1} X_{2} X_{3}$ as a subproduct. However, since

$$
d\left(F\left(E_{2} C X_{1} X_{2} X_{3}\right), \overline{F^{*}}\left(V_{2} C E_{1} C\right)\right)>2
$$

for $X_{1} X_{2} X_{3}=V_{k} E_{k} C(k=1,2,3), X_{1} X_{2}=E_{k} C(k=1,2), X_{1} X_{2} X_{3}=E_{k} V_{k} C(k=1,2), X_{1}=$ $C$, we conclude by Theorem 6.4 that $X_{1}=E_{3}$. Consequently, by Theorem 6.4 and Lemma 6.10 only $X_{2} X_{3}=V_{3} C$ remains as a possibility. However, using congruence, we can exclude this by

$$
d\left(F\left(E_{2} V_{2} C\right), \overline{F^{*}}\left(C E_{1} V_{1} C\right)\right)>2 .
$$

LEMMA 6.12. The regular double chain $\Pi T_{n}$ does not contain any of the following products as a subproduct,

$$
C E_{j} C E_{k} C \quad(j \neq k)
$$

Proof. Using congruence, we need only exclude $W_{3} W_{2} W_{1} C E_{1} C E_{2} C U_{1} U_{2} U_{3}$ as a subproduct. Since

$$
d\left(F\left(E_{2} C U_{1} U_{2} U_{3}\right), \overline{F^{*}}\left(C E_{1} C\right)\right)>2
$$


for $U_{1} U_{2} U_{3}=V_{k} E_{k} C(k=1,2,3), U_{1} U_{2}=E_{k} C(k=1,2), U_{1} U_{2} U_{3}=E_{k} V_{k} C(k=1,2)$, and $U_{1}=C$ is excluded by Lemmas $6.8,6.9$, we conclude by Theorem 6.4 that $U_{1}=E_{3}$; analogously $W_{1}=E_{3}$. Since also

$$
d\left(F\left(E_{2} C E_{3} V_{3} C\right), \overline{F^{*}}\left(C E_{1} C E_{3} C\right) \cup \overline{F^{*}}\left(C E_{1} C E_{3} V_{3} C\right)\right)>2,
$$

it follows by Theorem 6.4 that only $U_{2}=C$ and analogously $W_{2}=C$ remains as a possibility.

Repeating the argument in both directions, we find that $\Pi T_{n} \equiv \overleftarrow{C E_{1} C E_{2} C} \vec{E}_{3}$ is the only possibility left. However, this is also excluded, since

$$
K\left(\overleftarrow{C E_{1} C E_{2} C E_{3}}\right)=\sqrt[4]{17}>2
$$

LEMMA 6.13. The regular double chain $\Pi T_{n}$ does not contain any of the following products as a subproduct,

$$
E_{j} C^{2 m-1} V_{j}, \quad V_{j} C^{2 m-1} E_{j} \quad(m \in \mathbf{N})
$$

Proof. Using congruence, we need only by Theorem 6.4 exclude $E_{1} C^{2 m-1} V_{1} E_{1} C^{2 s-1}$ $X_{1} X_{2} X_{3}$ with $X_{1} \neq C$ as a subproduct.

It is easily proved by means of Lemma 6.16 below that in order to have

$$
d\left(F\left(V_{1} E_{1} C^{2 s-1} X_{1} X_{2} X_{3}\right), \overline{F^{*}}\left(C^{2 m-1} E_{1}\right)\right)<2,
$$

we must have $X_{1} \neq E_{1}, X_{1} X_{2} X_{3} \neq V_{k} E_{k} C(k=2,3)$ and $s<m$. By Theorem 6.4 we must have $X_{1} \notin\left\{E_{1}, V_{2}, V_{3}\right\}$.

For $1<s<m$ it follows similarly that in order to have

$$
d\left(F^{\prime}\left(C^{2 s-2} X_{1} X_{2} X_{3}\right), \overline{H^{* *}}\left(C E_{1} V_{1} C^{2 m-1} E_{1}\right)\right)<2,
$$

we must have $X_{1} \neq V_{1}, X_{1} X_{2} X_{3} \neq E_{k} V_{k} C(k=2,3)$. By Theorem 6.4 we must have $X_{1} \notin\left\{V_{1}\right.$, $\left.E_{2}, E_{3}\right\}$ in this case.

For $s=1$ it follows from

$$
d\left(F\left(V_{1}\right), \overline{F^{*}}\left(C E_{1}\right)\right)=2,
$$

that $X_{1} \neq V_{1}$, and hence necessarily $X_{1}=E_{k}(k=2,3)$. By Lemma 6.10, it follows that $X_{2} \neq C$, and consequently by Theorem 6.4 we must have $X_{1} X_{2} X_{3}=E_{k} V_{k} C(k=2,3)$. However, this possibility is excluded by

$$
d\left(F\left(E_{k} V_{k} C\right), \overline{F^{*}}\left(C E_{1} V_{1} C\right)\right)>2 \text { for } k \in\{2,3\}
$$

LEM M 6.14 . The regular double chain $\Pi T_{n}$ does not contain any of the following products as a subproduct,

$$
V_{j} E_{j} C^{2 m-1} E_{k} V_{k} \quad(j \neq k)
$$


Proof. Using congruence, we need only by Theorem 6.4 exclude

$$
C V_{2} E_{2} C^{2 m-1} E_{1} V_{1} C^{\rho-1} X_{1} X_{2} X_{3}
$$

with $X_{1} \neq C$ as a subproduct.

It is easily proved by means of Lemma 6.16 below that in order to have

$$
d\left(F\left(C^{2 m-2} E_{2} V_{2} C\right), \overline{F^{*}}\left(C E_{1} V_{1} C^{2 s-1} X_{1} X_{2} X_{3}\right)\right)<2,
$$

we must have $X_{1} \neq V_{1}, X_{1} X_{2} X_{3} \neq E_{k} V_{k} C(k=2,3)$, and further $X_{1} X_{2} X_{3} \neq V_{k} E_{k} C(k=2,3)$ in case $s \geqslant m-1$.

Similarly, in order to have

$$
d\left(F\left(V_{1} E_{1} C^{2 m-1} E_{2} V_{2} C\right), \overline{F^{*}}\left(C^{2 s-1} X_{1} X_{2} X_{3}\right)\right)<2,
$$

we must have $X_{1} X_{2} X_{3} \neq V_{k} E_{k} C(k=2,3)$ in case $s<m-1$.

Finally, $X_{1} \neq E_{1}$ by Lemma 6.13 , and $X_{1} X_{2} \neq E_{k} C(k=2,3)$ by Lemmas $6.8,6.9$, 6.12 .

Now the result follows by Theorem 6.4 .

LEM M 6.15. The regular double chain $\Pi T_{n}$ does not contain any of the following products as a subproduct,

$$
E, V_{j} C^{2 m-1} V_{k} E_{k} \quad(j \neq k) .
$$

Proof. Using congruence, we need only by Theorem 6.4 exclude

$$
C E_{2} V_{2} C^{2 m-1} V_{1} E_{1} C^{2 s-1} X_{1} X_{2} X_{3}
$$

with $X_{1} \neq C$ as a subproduct.

It is easily proved by means of Lemma 6.16 below that in order to have

$$
d\left(F\left(V_{1} E_{1} C^{2 s-1} X_{1} X_{2} X_{3}\right), \overline{F^{*}}\left(C^{2 m-1} V_{2} E_{2} C\right)\right)<2,
$$

we must have $X_{1} \neq E_{1}, X_{1} X_{2} X_{3} \neq V_{k} E_{k} C(k=2,3)$. Since $X_{1} \neq V_{1}$ by Lemma 6.13 , it follows by Theorem 6.4 that either $X_{1} X_{2}=E_{k} C(k=2,3)$ or $X_{1} X_{2} X_{3}=E_{k} V_{k} C(k=2,3)$.

However, the first possibility is excluded by Lemmas $6.8,6.9,6.10$, and the second possibility is excluded by Lemma 6.14.

LEMMA 6.16. For $r \in \mathbf{N}$ we have

where

$$
C^{r}=\left(\begin{array}{cc}
1 & -1+i \\
1-i & i
\end{array}\right)^{r}=\frac{1}{\sqrt{12}}\left(\frac{1+i}{2}\right)^{r-2}\left(\begin{array}{cc}
\frac{1}{2} \alpha_{r}+i\left(\frac{1}{2} \alpha_{r}+\alpha_{r-1}\right) & -\alpha_{r} \\
\alpha_{r} & -\frac{1}{2} \alpha_{r}+i\left(\frac{1}{2} \alpha_{r}+\alpha_{r-1}\right)
\end{array}\right)
$$

$$
\alpha_{r}=(1+\sqrt{\mathbf{3}})^{r}-(1-\sqrt{\mathbf{3}})^{r} .
$$

Proof. Since $\alpha_{r+1}=2 \alpha_{r}+2 \alpha_{r-1}$, we obtain the formula by induction on $r$.

LEMMA 6 17. $K\left(\overleftarrow{V_{1}} E_{1} C^{r} V_{3} E_{3} C^{r}\right)>2$ for all $r \in \mathbf{N}$. 
Proof. A computation of $V_{1} E_{1} C^{r} V_{3} E_{3} C^{r}$ by means of Lemma 6.16 shows that the regular double chain $\widetilde{V}_{1} \vec{E}_{1} C^{r} V_{3} E_{3} \vec{C}^{r}$ is associated to the quadratic form

$$
\begin{aligned}
\Phi= & \left(-\frac{1}{2} \alpha_{r}^{2}+\alpha_{r} \alpha_{r-1}+\alpha_{r-1}^{2}-i\left(5 \alpha_{r}^{2}+6 \alpha_{r} \alpha_{r-1}+2 \alpha_{r-1}^{2}\right),\right. \\
& -\frac{3}{2} \alpha_{r}^{2}-\alpha_{r} \alpha_{r-1}-\alpha_{r-1}^{2}+i\left(6 \alpha_{r}^{2}+4 \alpha_{r} \alpha_{r-1}\right), \\
& \left.+\frac{3}{2} \alpha_{r}^{2}-\alpha_{r} \alpha_{r-1}-\alpha_{r-1}^{2}-i\left(6 \alpha_{r}^{2}+8 \alpha_{r} \alpha_{r-1}+2 \alpha_{r-1}^{2}\right)\right) .
\end{aligned}
$$

Hence by further computations,

$$
\begin{gathered}
D=D(\Phi)=\left(\frac{19}{2} \alpha_{r}^{2}+13 \alpha_{r} \alpha_{r-1}+5 \alpha_{r-2}^{2}\right)^{2}-9 \cdot 4^{r+1} \\
\Phi(-1 ; i)=4 \alpha_{r}^{2}+6 \alpha_{r} \alpha_{r-1}+2 \alpha_{r-1}^{2}+i\left(\frac{5}{2} \alpha_{r}^{2}+3 \alpha_{r} \alpha_{r-1}+\alpha_{r-1}^{2}\right), \\
D-4 N(\Phi(-1, i))=9 \cdot 4^{r} .
\end{gathered}
$$

Consequently it follows by Theorem $6.3 \mathrm{~B}$, since $\mu=\mu(\Phi) \leqslant|\Phi(-1, i)|$, that

$$
K\left(\overleftrightarrow{V_{1}} E_{1} C^{r} V_{3} E_{3} \vec{C}^{r}\right) \geqslant \sqrt{\frac{D}{N(\Phi(-1, i))}}=\sqrt{4+\frac{9 \cdot 4^{r}}{N(\Phi(-1, i))}}>2 .
$$

LEMMA 6.18. The regular double chain $\Pi T_{n}$ does not contain any of the following products as a subproduct,

$$
V_{j} E_{j} C^{2 m-1} V_{k} E_{k}, \quad E_{k} V_{k} C^{2 m-1} E_{j} V_{j} \quad(j \neq k)
$$

Proof. Using congruence, we need only by Theorem 6.4 exclude

$$
C V_{3} E_{3} C^{2 m-1} V_{1} E_{1} C^{2 s-1} X_{1} X_{2} X_{3}
$$

with $X_{1} \neq C$ as a subproduct.

It is easily proved by means of Lemma 6.16 that in order to have

$$
d\left(F\left(C^{2 s-2} X_{1} X_{2} X_{3}\right), \overline{F^{*}}\left(C E_{1} V_{1} C^{2 m-1} E_{3} V_{3} C\right)\right)<2,
$$

we must have $X_{1} \neq V_{1}, X_{1} X_{2} \neq E_{k} C(k=2,3), X_{1} X_{2} X_{3} \neq E_{k} V_{k} C(k=2,3)$; hence by Theorem 6.4, $X_{1} \notin\left\{V_{1}, E_{2}, E_{3}\right\}$.

Similarly, in order to have

$$
d\left(F\left(E_{2} V_{2} C^{2 m-1} E_{1} V_{1} C\right), \overline{F^{*}}\left(C^{2 s-1} Y_{1} Y_{2} Y_{3}\right)\right)<2,
$$

we must have $Y_{1} \neq V_{3}, Y_{1} Y_{2} \neq E_{j} C(j=1,2), Y_{1} Y_{2} Y_{3} \neq E_{j} V_{j} C(j=1,2)$, and further $s \leqslant m$ in case $Y_{1} Y_{2} Y_{3}=V_{1} E_{1} C$. Using congruence, we conclude by Theorem 6.4 that $X_{1} \notin\left\{V_{2}, E_{3}, E_{1}\right\}$, and that $s \leqslant m$ in case $X_{1} X_{2} X_{3}=V_{3} E_{3} C$. 
Repeating the argument above, we see that there exists a number $l \in \mathbf{N}, l \leqslant m$, such that

$$
\prod T_{n} \equiv \ldots \widehat{V_{1} E_{1} C^{2 l-1}} \overline{V_{3} E_{3} C^{2 i}=1} .
$$

However, this possibility is excluded by Lemma 6.17.

Combining Theorem 6.4 and Lemmas 6.8, 6.9, 6.10,6.11, 6.12, 6.14, 6.15, 6.18, we obtain the following important improvement of Theorem 6.4 .

ТНЕОВЕM 6.5. Suppose that the regular double chain $\Pi T_{n}$ satisfies the preliminary restrictions of Theorem 6.4. Then $\Pi T_{n}$ is congruent to

$$
\ldots C^{2 m_{-1}-1} T_{1}^{(-1)} \ldots T_{k_{-1}}^{(-1)} C^{2 m_{0}-1} T_{1}^{(0)} \ldots T_{k_{0}}^{(0)} C^{2 m_{1}-1} \ldots
$$

where $m_{r} \in \mathbf{N}$ for all $r \in \mathbf{Z}$ and $T_{1}^{(r)} \ldots T_{k_{r}}^{(r)}$ for each:r $\in \mathbf{Z}$ is one of the three products

$$
E_{1}, V_{1} E_{1}, E_{1} V_{1}
$$

\subsection{The main theorem on the minimum of quadratic forms}

THEOREM 6.6. Let $\Phi=(A, B, C)$ be a complex quadratic form with discriminant $D=D(\Phi) \neq 0$ and minimum $\mu=\mu(\Phi)$.

A. If

$$
\sqrt{|D|} / \mu<2
$$

then $\Phi$ is equivalent to a multiple of either

$$
G=(-3 i, 3 i, 1-2 i), \bar{G}=(3 i,-3 i, 1+2 i)
$$

or some $G_{\Lambda}$, where

$$
G_{\Lambda}(X, Y)=g_{\Lambda}(2 X-Y, i Y),
$$

the forms $g_{\Lambda}$ being the C-minimal forms of chapter 5 .

B. Conversely (6.17) holds for all forms equivalent to a multiple of $G, \bar{G}$ or some $G_{\Lambda}$; specifically

$$
\begin{gathered}
\sqrt{|D(G)|} / \mu(G)=\sqrt{|D(\bar{G})|} / \mu(\bar{G})=\sqrt{\frac{3}{5} \sqrt{41}} \\
\sqrt{\left|D\left(G_{\Lambda}\right)\right|} / \mu\left(G_{\Lambda}\right)=\sqrt{4-\Lambda^{-2}}
\end{gathered}
$$

C. There are $2^{\aleph_{0}}$ forms $\Phi$, none of which are equivalent to a multiple of any other, such that

$$
\sqrt{\bar{D}(\Phi) \mid} / \mu(\Phi)=2
$$

Proof. To prove part A we notice, that $\Phi$ must have both roots inequivalent to real 
numbers, since otherwise $\sqrt{|D|} / \mu \geqslant \sqrt{5}$ by the Markoff-Hurwitz approximation theorem. Let $\Pi T_{n}$ by any regular double chain associated to $\Phi$. By Theorem $6.3 \mathrm{~A}$, we must have

$$
K\left(\Pi T_{n}\right) \leqslant \sqrt{|\bar{D}|} / \mu<2,
$$

and hence by Theorems $6.4,6.5$ either

$$
\text { I. } \begin{aligned}
\Pi T_{n} & =\stackrel{C}{C V_{1} E_{1} V_{1} C E_{3} C V_{2} E_{2} V_{2} C E_{1} C V_{3} E_{3} V_{3} C E_{2}}, \\
\Pi T_{n} & =\overleftarrow{C} V_{1} E_{1} V_{1} C E_{2} C V_{3} E_{3} V_{3} C E_{1} C V_{2} E_{2} V_{2} C E_{3},
\end{aligned}
$$

or

II. ПI $T_{n} \equiv \Pi U_{n}$, where $U_{n} \in\left\{V_{1}, E_{1}, C\right\}$ for all $n \in \mathbf{Z}$.

Case I. (The asymmetric case.) It follows by Example 6.3 that $\Phi$ is equivalent to a multiple of $G, \bar{G}$, respectively. Notice that $G, \bar{G}$ are inequivalent, since $D(G)=15+12 i \neq$ $\pm D(\bar{G})= \pm(15-12 i)$.

Case II. (The symmetric case.) Then $\Phi$ is equivalent to a form, where the roots $\xi, \eta$ satisfy $\operatorname{Re} \xi=\operatorname{Re} \eta=\frac{1}{2}$ (cf. chapter 4 ), hence $\Phi$ is equivalent to a multiple of a form $F$, where

$$
F(X, Y)=f(2 X-Y, i Y)
$$

where $f=(\alpha, \beta, \gamma)$ is a real form with $\delta(f)>0$ and having irrational roots. Notice that $F$ also satisfies $(6.17)$, and that $D(F)=-4 \delta(f)$.

If $f$ is not $C$-equivalent to a multiple of some $g_{\Lambda}$ or $h_{\mathrm{M}}$, then $\sqrt{\delta(f)} / v(f) \geqslant 2$ by Theorem $5.3 \mathrm{~A}$, hence either there exists $\left(x_{0}, y_{0}\right) \in \mathbf{Z}_{0}^{2}$, such that

$$
\sqrt{\delta(f)} /\left|f\left(x_{0}, y_{0}\right)\right|>\sqrt{|\overline{D(F)}|} / \mu(F)=2 \sqrt{\delta(f)} / \mu(F),
$$

of there exists $\left(x_{1}, y_{1}\right) \in \mathbf{Z}_{1}^{2}$, such that

$$
\sqrt{\delta(f)} /\left|2 f\left(x_{1}, y_{1}\right)\right|>\sqrt{|\overrightarrow{D(F)}|} / \mu(F)=2 \sqrt{\delta(f)} / \mu(F) .
$$

Consequently, either

$$
\mu(F)>2\left|f\left(x_{0}, y_{0}\right)\right|=\left|f\left((1+i) x_{0},(1+i) y_{0}\right)\right|=\left|F\left(\frac{i x_{0}+y_{0}}{1+i},(1-i) y_{0}\right)\right|
$$

or

$$
\mu(F)>4\left|f\left(x_{1}, y_{1}\right)\right|=\left|f\left(2 x_{1}, 2 y_{1}\right)\right|=\left|F\left(x_{1}-i y_{1},-2 i y_{1}\right)\right|,
$$

in contradiction to the definition of $\mu(F)$, since $\left(i x_{0}+y_{0}\right) /(1+i) \in \mathbf{Z}[i]$.

Suppose that $f \approx \operatorname{ch}_{M_{2}}(c \neq 0)$, where $\left(\Lambda_{1}, \Lambda_{2} ; M_{1}, M_{2}\right)$ is any non-singular solution of equation (5.4) with $\Lambda_{1}<\Lambda_{2}, \mathrm{M}_{1}<\mathrm{M}_{2}$, and associated quintuple $\left(\varepsilon ; l_{1}, l_{2} ; m_{1}, m_{2}\right)$. Then $F \sim c H_{\mathrm{M}_{2}}$, where $H_{\mathrm{M}_{2}}(X, Y)=h_{\mathrm{M}_{2}}(2 X-Y, i Y)$. 
It follows easily by Lemma 5.6, that in case $\varepsilon=1$, we have

$$
\left|\mathrm{M}_{2} H_{\mathrm{M}_{2}}\left(\frac{1}{2}\left(\Lambda_{1}-1+l_{1} \mathrm{i}\right), \Lambda_{1}\right)\right|=\Lambda_{1} \sqrt{16 \mathrm{M}_{2}^{2}-8} .
$$

Also, it follows by Lemma 5.6 (vii) (ix), that $\Lambda_{1}$ and $1-l_{1} i$ have a common divisor $\varrho$ (in $\mathbf{Z}[i])$ with $N(\varrho)=\Lambda_{1}$, in particular $\left(\Lambda_{1}-1+l_{1} i\right) / \varrho \in Z[i]$; however, since $N\left(\left(\Lambda_{1}-1+l_{1} i\right) / \varrho\right)$ $=\left(\Lambda_{1}^{2}-2 \Lambda_{1}+1+l_{1}^{2}\right) / \Lambda_{1}=\Lambda_{1}-2+\lambda_{1} \equiv 1-2+1=0 \quad(\bmod 4)$ by Lemma 5.6 (vii), also $\left(\Lambda_{1}-1+l_{1} i\right) /(2 \varrho) \in \mathbf{Z}[i]$.

Consequently, for $M=M_{2}$ and $\varepsilon=1$, we have

$$
\mu\left(\mathbf{M} H_{\mathrm{M}}\right) \leqslant \sqrt{\mathbf{1 6 \mathbf { M } ^ { 2 } - 8}} .
$$

In case $M=M_{2}$ and $\varepsilon=-1$, we can prove (6.19) similarly, using that

$$
\left|\mathrm{M}_{2} H_{\mathrm{M}_{2}}\left(\frac{1}{2}\left(\Lambda_{1}-1+\left(l_{1}-4 \Lambda_{1}\right) i\right), \Lambda_{1}\right)\right|=\Lambda_{1} \sqrt{16 \mathrm{M}_{2}^{2}-8} .
$$

Finally, (6.19) is valid for $M=1$, since

$$
H_{1}(X, Y)=h_{1}(2 X-Y, i Y)=2(1-i)\left((1+i) X^{2}-2 X Y+Y^{2}\right) .
$$

Using (5.7) and (6.19), we see that in case $f \approx c h_{\mathrm{M}}$ (and hence $F \sim c H_{\mathrm{M}}$ ), we have

$$
\sqrt{|D(F)|} / \mu(F)=\sqrt{\left|D\left(\mathbf{M} H_{\mathrm{M}}\right)\right|} / \mu\left(\mathrm{M} H_{\mathrm{M}}\right)=2 \sqrt{\delta\left(\mathrm{M} h_{\mathrm{M}}\right)} / \mu\left(\mathbf{M} H_{\mathrm{M}}\right) \geqslant 2 .
$$

This completes the proof of part $A$.

Part B. It follows from Example 6.4 that

$$
\sqrt{|\bar{D}(\bar{G})|} \mid / \mu(\bar{G})=\sqrt{|D(G)|} / \mu(G)=\sqrt{\frac{3}{5} \sqrt{41}} .
$$

Also it follows from (5.7) that

$$
\sqrt{\mid D\left(\Lambda G_{\Lambda}\right)} \mid=2 \sqrt{\delta\left(\Lambda g_{\Lambda}\right)}=4 \Lambda \sqrt{4-\Lambda^{-2}}
$$

hence to prove $B$ we need only show that

$$
\mu\left(\frac{1}{4} \Lambda G_{\Lambda}\right)=\Lambda .
$$

By Lemma 5.6, the form

$$
\begin{aligned}
\frac{1}{4} \Lambda G_{\Lambda}(X, Y)=\Lambda X^{2}-(\Lambda-i(2 \Lambda-l)) X Y+ & ((\Lambda-\hat{\lambda}) / 4+l-i(\Lambda-l / 2)) Y^{2} \\
& =\Lambda\left(X-\frac{1}{2}\left(1+i \vartheta_{1}\left(\mathrm{~g}_{\Lambda}\right)\right) Y\right)\left(X-\frac{1}{2}\left(1+i \vartheta_{2}\left(\mathrm{~g}_{\Lambda}\right)\right) Y\right)
\end{aligned}
$$

has coefficients in $\mathrm{Z}[i]$, and it follows easily from Theorem $5.3 \mathrm{~B}$ by considering the two cases $\operatorname{Re}(X / Y)=\frac{1}{2}$ and $\operatorname{Re}(X / Y) \neq \frac{1}{2}$ separately, that

$$
\frac{1}{4}\left|D\left(\frac{1}{4} \Lambda G_{\Lambda}\right)\right|=\Lambda^{2}-\frac{1}{4} \leqslant N\left(\mu\left(\frac{1}{4} \Lambda G_{\Lambda}\right)\right) \leqslant \Lambda^{2}=N\left(\frac{1}{4} \Lambda G_{\Lambda}(1,0)\right) .
$$

This yields $(6.20)$, since $N\left(\mu\left(\frac{1}{4} \Lambda G_{\Lambda}\right)\right) \in \mathbf{Z}$. 
Part $\mathrm{C}$ follows similarly from Theorem 5.3 C by considering forms $F$, where $F(X ; Y)=$ $f(2 X-Y, i Y)$, and $f$ is any form with $\sqrt{\delta(f)} / v(f)=2$.

\subsection{The main theorem on the approximation constant}

THEOR M 6.7 . Let $\xi \in \mathbf{C} \backslash Q(i)$.

A. If

$$
C(\xi)<2,
$$

then $\xi$ is equivalent to a root of either $G, \vec{G}$ or some $G_{\Lambda}$.

B. Conversely if $\xi$ is equivalent to a root of $G$ or $\bar{G}$, then

$$
C(\xi)=\sqrt{\frac{3}{5} \sqrt{41}}
$$

and if $\xi$ is equivalent to a root of $G_{\Lambda}$, then

$$
C(\xi)=\sqrt{4-\Lambda^{-2}}
$$

C. There are $2^{\aleph_{0}}$ different equivalence classes of $\xi$ such that $C(\xi)=2$.

Remark. Since no isolation theorem (like Theorem I, chapter II of [4] or Theorem 5.1) is available for complex forms, Theorem 6.7 cannot be deduced from Theorem 6.6 (cf. the proofs of Theorem III, chapter II of [4] or Theorem 5.4).

Proof. Suppose $\xi \in \mathbf{C} \backslash \mathbf{Q}(i)$ with $C(\xi) \leqslant 2$. Then $\xi$ is inequivalent to a real number, since otherwise $C(\xi) \geqslant \sqrt{5}$ by the theorem of Hurwitz ([19]). Hence by Theorem $2.2, \xi=\xi_{0}$ has a unique regular chain

$$
\operatorname{ch} \xi=T_{1} T_{0} \ldots T_{n} \ldots
$$

Also by Theorem 2.6

$$
C(\xi)=\lim \sup c_{j}^{(n)}
$$

where $c_{j}^{(n)}$ for $1 \leqslant j \leqslant 3$ is given by $(2.9),(2.10),(2.11)$.

By putting

$$
\zeta_{n+1}=-q_{2}^{(n)} / q_{1}^{(n)}
$$

we may rewrite the $c_{j}^{(n)}$ as follows,

$$
c_{1}^{(n)}=\left|\xi_{n+1}-\zeta_{n+1}\right|, c_{2}^{(n)}=\left|\xi_{n+1}^{-1}-\zeta_{n+1}^{-1}\right|, c_{3}^{(n)}=\left|\left(\xi_{n+1}-1\right)^{-1}-\left(\zeta_{n+1}-1\right)^{-1}\right|
$$

From

$$
T_{0} T_{1} \ldots T_{n}=\left(\begin{array}{ll}
p_{1}^{(n)} & p_{2}^{(n)} \\
q_{1}^{(n)} & q_{2}^{(n)}
\end{array}\right)
$$

6-752903 Acta mathematica 134. Imprimé le 30 Juillet 1975 
we get by taking inverses and using Lemma 1.1 (iv), that

$$
\left(\begin{array}{cc}
q_{2}^{(n)} & -p_{2}^{(n)} \\
-q_{1}^{(n)} & p_{1}^{(n)}
\end{array}\right)= \pm \varepsilon_{n} \overline{T_{n}} \overline{\ldots T_{1} T_{0}} .
$$

Consequently for any $h, k \in \mathbf{N}(k \leqslant n)$, we have

$$
\begin{aligned}
& \xi_{n+1}=\left[T_{n+1} T_{n+2} \ldots\right] \in\left\{\begin{array}{ll}
F\left(T_{n+1} \ldots T_{n+h}\right) & \text { if } \varepsilon_{n}= \pm 1 \\
F^{*}\left(T_{n+1} \ldots T_{n+h}\right) & \text { if } \varepsilon_{n}= \pm i
\end{array},\right. \\
& \zeta_{n+1}=-q_{2}^{(n)} / q_{1}^{(n)} \in \begin{cases}\bar{F}\left(T_{n} \ldots T_{n-k}\right) & \text { if } \varepsilon_{n}= \pm i \\
\overline{F^{*}}\left(T_{n} \ldots T_{n-k}\right) & \text { if } \quad \varepsilon_{n}= \pm 1\end{cases}
\end{aligned}
$$

Because of the resemblance between the formulae for

$$
C\left(\left[T_{0} T_{1} \ldots T_{n} \ldots\right]\right) \text { and } K\left(\Pi T_{n}\right)
$$

it is obvious, that the content of section 6.3 can be modified to yield the following result:

If $C(\xi)<2$, then either

I. $\operatorname{ch} \xi=T_{0} \ldots T_{h} \overline{C V_{1} E_{1} V_{1} C E_{3} C V_{2} E_{2} V_{2} C E_{1} C V_{3} E_{3} V_{3} C E_{2}}$

$$
\operatorname{ch} \xi=T_{0} \ldots T_{h} \overparen{C V_{1} E_{1} V_{1} C E_{2} C V_{3} E_{3} V_{3} C E_{1} C V_{2} E_{2} V_{2} C E_{3}} \text {, }
$$

or

II. $\xi \sim \eta=\left[U_{0} U_{1} \ldots U_{n} \ldots\right]$, where $U_{n} \in\left\{V_{1}, E_{1}, C\right\}$ for all $n \in \mathbf{N}_{0}$.

Case I. (The asymmetric case.) By Example 6.3 and Theorems 2.2, 2.4, $\xi$ is necessarily equivalent to a root of either $G$ or $\bar{G}$. Conversely if $\xi$ is equivalent to a root of either $G$ or $\bar{G}$, then by Theorem 2.7 and Theorem 6.6 , we have

$$
C(\xi)=\sqrt{\frac{3}{5} \sqrt{41}}
$$

Case II. (The symmetric case.) Then (ef. chapter 4) necessarily $\xi \sim \frac{1}{2}(1+i \alpha)$, where $\alpha \in \mathbf{R} \backslash \mathbf{Q}$. For convenience we put

$$
C_{1}\left(\frac{1}{2}(1+i \alpha)\right)=\lim \sup \left(|q|\left|\frac{1}{2}(1+i \alpha) q-p\right|\right)^{-1},
$$

the $\lim$ sup being extended over all $p, q \in \mathbf{Z}[i](q \neq 0)$ with $\operatorname{Re}(p / q)=\frac{1}{2}$.

Similarly we put

$$
C_{2}\left(\frac{1}{2}(1+i \alpha)\right)=\lim \sup \left(|q|\left|\frac{1}{2}(\mathrm{I}+i \alpha) q-p\right|\right)^{-1},
$$

the $\lim$ sup being extended over all $p, q \in \mathbf{Z}[i](q \neq 0)$ with $\operatorname{Re}(p / q) \neq \frac{1}{2}$. 
We claim that for any $\alpha \in \mathbf{R} \backslash \mathbf{Q}$, we have

$$
C_{1}\left(\frac{1}{2}(1+i \alpha)\right)=d(\alpha)
$$

where $d(\alpha)$ is the $C$-approximation constant of $\alpha$, and

$$
C_{2}\left(\frac{1}{2}(1+i \alpha)\right) \leqslant 2
$$

Suppose first that $\pi / \varrho \in Q_{0}$ (cf. section 4.2), then

$$
\frac{1}{2}(1+i(\pi / \varrho))=(\varrho+\pi i) /(2 \varrho),
$$

where

$$
N(\varrho+\pi i)=\pi^{2}+\underline{Q}^{2} \equiv 2(\bmod 4), N(2 \varrho)=2 \cdot 2 \varrho^{2} .
$$

By putting $p=(\varrho+\pi i) /(1-i)$ and $q=\varrho(1+i)$, we obtain, since ged $(\pi, \varrho)=1$, that

$$
p, q \in \mathbf{Z}[i], \operatorname{gcd}(p, q)=1, \frac{1}{2}(1+i(\pi / \varrho))=p / q
$$

Also

$$
\left(|q|\left|\frac{1}{2}(1+i \alpha) q-p\right|\right)^{-1}=(|\varrho||\alpha \varrho-\pi|)^{-1}
$$

Suppose next that $\pi / \varrho \in \mathbf{Q}_{1}$, then

$$
\frac{1}{2}(1+i(\pi / \varrho))=(\varrho+\pi i) /(2 \varrho),
$$

where

$$
N(\varrho+\pi i)=\pi^{2}+\varrho^{2} \equiv 1(\bmod 4), N(2 \varrho)=4 \varrho^{2} .
$$

By putting $p=\varrho+\pi i$ and $q=2 \varrho$, we obtain, since $\operatorname{gcd}(\pi, \varrho)=1$, that

$$
p, q \in \mathbf{Z}[i], \operatorname{gcd}(p, q)=1, \frac{1}{2}(1+(\pi / \varrho))=p / q \text {. }
$$

Also

$$
\left(|q|\left|\frac{1}{2}(1+i \alpha) q-p\right|\right)^{-1}=\frac{1}{2}(|\varrho||\alpha \varrho-\pi|)^{-1}
$$

Altogether this proves $(6.22)$.

If $\operatorname{Re}(p / q) \neq \frac{1}{2}$ and $p, q \in \mathbf{Z}[i]$, then obviously $p / q=a+i b$ with $a, b \in \mathbf{Q}$, and $\left|a-\frac{1}{2}\right| \geqslant$ $1 /\left(2|q|^{2}\right)$. This proves (6.23).

It follows by $(6.22)$ that if $\xi \sim \frac{1}{2}(1+i \alpha)$ has $C(\xi)<2$, then necessarily $d(\alpha)<2$. By Theorem 5.4 A, $\alpha$ must be a root of some $g_{\Lambda}$ or $h_{\mathrm{M}}$, hence $\frac{1}{2}(1+i \alpha)$ must be a root of some $G_{\Lambda}$ or $H_{\mathrm{M}}$. What remains to be proved of parts $\mathrm{A}$ and $\mathrm{B}$ follows thus by Theorems 2.6 and 6.6.

It follows from $(6.22),(6.23)$ and Theorem 5.4 $\mathrm{C}$ that $C\left(\frac{1}{2}(1+i \alpha)\right)=2$ for any $\alpha$ with $d(\alpha)=2$. This proves part $\mathrm{C}$. 


\section{References}

[1]. Aunic, A., Essai sur la théorie des fractions continues. J. Math. Pures Appl., (5) 8 (1902), $387-431$.

[2]. Casseis, J. W. S., The Markoff chain. Ann. of Math., 50 (1949), 676-685.

[3]. - Über einen Perronschen Satz. Arch. Math., (Basel) 3 (1952), 10-14.

[4]. - An introduction to diophantine approximation. Cambridge University Press, Cambridge, 1957.

[5]. Cassels, J. W. S., Ledermann, W. \& Mahler, K., Farey section in $k(i)$ and $k(\varrho)$. Philos. Trans. Roy. Soc. London, ser. A 243 (1951), 585-626.

[6]. Cohs, H., Approach to Markoff's minimal forms through modular functions. Ann. of Math., (2) 61 (1955), 1-12.

[7]. - Representation of Markoff's binary quadratic forms by geodesics on a perforated torus. Acta Arith., 18 (1971), 125-136.

[8]. Drckson, L. E., Introduction to the theory of numbers. The University of Chicago Pross, Chicago 1929; reprinted by Dover, New York, 1957.

[9]. - Studies in the theory of numbers. The University of Chicago Press, Chicago 1980; reprinted by Chelsea, New York, 1957.

[10]. Dirichuet, P. G. L., Recherches sur les formes quadratiques a coefficients et a indéterminées complexes. J. Reine Angew. Math., 24 (1842), 291-371.

[11]. Eggan, L. C., On diophantine approximations. Trans. Amer. Math. Soc., 99 (1961), 102117.

[12]. Ford, L. R., Rational approximations to irrational complex numbers. Trans. Amer. Math. Soc., 19 (1918), 1-42.

[13]. - On the closeness of approach of complex rational fractions to a complex irrational number. Trans. Amer. Math. Soc., 27 (1925), 146-154.

[14]. - Fractions. Amer. Math. Monthly, 45 (1938), 586-601.

[15]. Frobenius, G., Über die Markoffschen Zahlen. S-B. Berlin. Math. Gesellsch., (1913), 458487.

[16]. Gauss, C. F., Disquisitiones arithmeticae. Leipzig, 1801.

[17]. Hall, M.; Jr., The Markoff spectrum. Acta Arith., 18 (1971), 387-399.

[18]. Hurwitz, A., Über die Entwicklung komplexer Grössen in Kettenbrüche. Acta Math., 11 (1887-88), 187-200.

[19]. - Über die angenäherte Darstellung der Irrationalzahlen durch rationale Brüche. Math. Ann., 39 (1891), 279-284.

[20]. Hunwitz, J., Über eine besondere Art der Kettenbruchentwickelung komplexer Grössen. Dissertation, Halle, 1895.

[21]. Lekkerkerken, C. G., Geometry of numbers. Wolters-Nordhoff Publishing, Groningen \& North-Holland Publishing Company, Amsterdam, 1969.

[22]. Leveque, W. J., Geometric properties of Farey sections in $k(i)$. Indag. Math., 14 (1952), 415-426.

[23]. - Continued fractions and approximations in $k(i)$, I-II. Indag. Math., 14 (1952), $526-545$.

[24]. Markorf, A., Sur les formes quadratiques binaires indéfinies. I, Math. Ann., 15 (1879), 381-406; II, Math. Ann., 17 (1880), 379-399.

[25]. Perron, O., Über die Approximation einer komplexen Zahl durch Zahlen des Körpers K(i). I, Math. Ann., 103 (1930), 533-544; II, Math. Ann., 105 (1931), 160-164.

[26]. - - Eine Abschätzung für die untere Grenze der absoluten Beträge der durch eine reelle oder imaginäre binäre quadratische Form darstellbaren Zahlen. Math. Z., 35 (1932), $563-578$. 
[27]. - Die Lehre von den Kettenbrüchen, Band I. Teubner, Stuttgart, 1954.

[28]. Portou, G., Sur l'approximation des nombres complexes par les nombres des corps imaginaires quadratiques dénués d'idéaux non principaux. Ann. Sci. École Norm. Sup., (3) 70 (1953), 199-265.

[29]. Remak, R., Ưber indefinite binäre quadratische Minimalformen. Math. Ann., 92 (1924), 155-182.

[30]. — Über die geometrische Darstellung der indefiniten binären quadratischen Minimalformen. Jber. Deutsch. Mat. - Verein, 33 (1925), 228-245.

[31]. Rosinson, R. M., The approximation of irrational numbers by fractions with odd or even terms. Duke Math. J., 7 (1940), 354-359.

[32]. Ryut-NARDzewski, C., On the ergodic theorems II. Studia Math., 12 (1951), 74-79.

[33]. Schmid, A. L., Farey triangles and Farey quadrangles in the complex plane. Math. Scand., 21 (1967), 241-295.

[34]. Scotr, W. T., Approximation to real irrationals by certain classes of rational fractions. Bull. Amer. Math. Soc., 46 (1940), 124-129.

[35]. DE VRoedt, C., Measure-theoretical investigations concerning continued fractions. Indag. Math., 24 (1962), 583-591.

[36]. VULAKH, L. YA., On Markov's spectrum of imaginary quadratic fields $\mathbf{Q}(i \sqrt{D})$, where $D \equiv 3$ (mod 4). Vestnik. Moskov. Univ. Ser. I Mat. Meh., 6 (1971), 32-41. (Russian).

Recieved May 24, 1974. 